\title{
An algebraic model for the loop space homology of a homotopy fiber
}

\author{
KATHRYN HESS \\ RAN LEVI
}

\begin{abstract}
Let $F$ denote the homotopy fiber of a map $f: K \rightarrow L$ of 2 -reduced simplicial sets. Using as input data the strongly homotopy coalgebra structure of the chain complexes of $K$ and $L$, we construct a small, explicit chain algebra, the homology of which is isomorphic as a graded algebra to the homology of $G F$, the simplicial (Kan) loop group on $F$. To construct this model, we develop machinery for modeling the homotopy fiber of a morphism of chain Hopf algebras.

Essential to our construction is a generalization of the operadic description of the category DCSH of chain coalgebras and of strongly homotopy coalgebra maps given by Hess, Parent and Scott [6] to strongly homotopy morphisms of comodules over Hopf algebras. This operadic description is expressed in terms of a general theory of monoidal structures in categories with morphism sets parametrized by co-rings, which we elaborate here.
\end{abstract}

55P35, 16W30; 18D50, 18G55, 55U10, 57T05, 57T25

\section{Introduction}

In this article we propose a "neoclassical" approach to computing the homology algebra of double loop spaces, based on developing a deep, operadic understanding of "strongly homotopy" structures for coalgebras and comodules, a notion that goes back more than 30 years, to work of Gugenheim, Halperin, Munkholm and Stasheff [5; 15]. We also make extended use of one-sided cobar constructions, which we apply in innovative ways.

Let Cotor $^{C}(-,-)$ denote the derived functor of the cotensor product $-\square_{C}-$, for any coalgebra $C$. Eilenberg and Moore proved long ago [3] that for any (Serre) fibration $E \rightarrow B$ with fiber $F$ such that $B$ is connected and simply connected and for any commutative ring $R$, there is an $R$-linear isomorphism

$$
\mathrm{H}_{*}(F ; R) \cong \operatorname{Cotor}^{C_{*}(B ; R)}\left(C_{*}(E ; R), R\right) .
$$


In particular, if $B$ is actually $2-$ connected, then

$$
\mathrm{H}_{*}(\Omega F ; R) \cong \operatorname{Cotor}^{C_{*}(\Omega B ; R)}\left(C_{*}(\Omega E ; R), R\right),
$$

so that for any 2-connected space $X$, there is a linear isomorphism

$$
\mathrm{H}_{*}\left(\Omega^{2} X ; R\right) \cong \operatorname{Cotor}^{C_{*}}(\Omega X ; R)(R, R) .
$$

The duals of [4, Theorems 4.1 and 5.1] imply that $\operatorname{Cotor}^{C_{*}(\Omega B ; R)}\left(C_{*}(\Omega E ; R), R\right)$ admits an algebra structure with respect to which the isomorphism (0-2) can be assumed to be an algebra morphism.

In this article we define a functor $\mathfrak{L F}$ that associates to any map $f$ of 2 -reduced simplicial sets a chain algebra $\mathfrak{L F}(f)$ such that $\mathrm{H}_{*}(\mathfrak{L F}(f))$ is isomorphic as an algebra to $\mathrm{H}_{*}(G F)$, where $F$ is the homotopy fiber of $f$ and $G$ is the Kan loop group functor on simplicial sets (Theorem 6.1). The algebra isomorphism $\mathrm{H}_{*}(\mathfrak{L F}(f)) \cong \mathrm{H}_{*}(G F)$ is realized on the chain level by a zig-zag of quasi-isomorphisms of chain algebras

$$
\mathfrak{L F}(f) \stackrel{\simeq}{\simeq} \stackrel{\simeq}{\longrightarrow} \ldots \stackrel{\simeq}{\longleftarrow} \stackrel{\simeq}{\longrightarrow} C(G F) .
$$

As a special case, we obtain a functor $\mathfrak{L}_{2}$ from the category of 2-reduced simplicial sets to the category of connected chain algebras over a principal ideal domain $R$ such that $\mathrm{H}_{*}\left(\mathfrak{L}_{2}(K)\right)$ is isomorphic as a graded algebra to $\mathrm{H}_{*}\left(G^{2} K ; R\right)$.

The model that we propose for the loop homology of a homotopy fiber offers certain advantages. First, there are no extension problems to be solved: the homology algebra of the model is exactly isomorphic to the homology algebra of the loops on the homotopy fiber. Second, our model is functorial, so that it can be applied to determining the homomorphism induced on double loop space homology by a simplicial map.

Finally, our model is "small" and therefore amenable to explicit computations. More precisely, if $K$ is a simplicial set with exactly $n$ nondegenerate simplices of positive degree, where $n<\infty$, then our model $\mathfrak{L}_{2}(K)$ of $G^{2} K$ is a subalgebra of a free algebra on $2 n$ generators. Like the differential in the cobar construction on $C(K)$, the differential in $\mathfrak{L}_{2}(K)$ depends only on the differential and the comultiplication on $C(K)$. In particular, in Section 6.2, we provide an explicit, relatively simple description of our model when $K$ is either formal or a double suspension.

By way of comparison, note that the iterated cobar construction on the chains on $K$, which is another model of $G^{2} K$, is free as an algebra on an infinite number of generators. Its differential depends not only on the differential and the comultiplication on $C(K)$, but also on the natural comultiplication on the cobar construction on $C(K)$, which can be very involved. Another possible model, the cobar construction on $C(G K)$, is also free, but on a generating set that is infinite in each degree, and, in addition, has 
a very complicated differential. Finally, both the multiplication and the differential on the chain Hopf algebra $C\left(G^{2} K\right)$ itself are extremely complex.

In the general case of loops on the homotopy fiber $F$ of a simplicial map $K \rightarrow L$, the dual of Theorem 5.1 in Félix-Halperin-Thomas [4] states that there is a quasiisomorphism of chain algebras

$$
C(G F) \stackrel{\simeq}{\rightarrow} C(G K) \otimes_{t_{\Omega}} \Omega C(G L),
$$

where $C(G K) \otimes_{t_{\Omega}} \Omega C(G L)$ denotes the one-sided cobar construction of Definition 3.1, endowed with the multiplication of Corollary 3.6. The chain algebra $C(G K) \otimes_{t_{\Omega}}$ $\Omega C(G L)$ is not of finite type, even if $K$ and $L$ have only a finite number of nondegenerate simplices, and both its differential and its multiplication are quite complicated. On the other hand, if $K$ and $L$ have exactly $m$ and $n$ nondegerate simplices of positive degree, respectively, then the chain algebra model constructed here, $\mathfrak{L} \mathfrak{F}(f)$, is a subalgebra of a free algebra on $m+2 n$ generators, so that its multiplicative structure is relatively simple. Its differential is also much easier to give explicitly than that of $C(G K) \otimes_{t_{\Omega}} \Omega C(G L)$.

To construct our models, we need the full Alexander-Whitney coalgebra structure of the normalized chains on a simpicial set. The category $\mathbf{F}$ of Alexander-Whitney coalgebras (cf Definition 2.12) was introduced and studied in Hess-Parent-Scott-Tonks [8]. The objects of $\mathbf{F}$ are connected chain coalgebras $(C, \Delta)$ such that the comultiplication $\Delta$ is itself a coalgebra map up to strong homotopy, ie up to a coherent, infinite family of homotopies, which we denote $\Psi$. Furthermore, there is a functor $\widetilde{\Omega}: \mathbf{F} \rightarrow \mathbf{H}$, where $\mathbf{H}$ is the category of chain Hopf algebras, such that the chain algebra underlying $\widetilde{\Omega}(C, \Psi)$ is $\Omega C$, the cobar construction on $C$.

An Alexander-Whitney model of a chain Hopf algebra $H$ consists of an AlexanderWhitney coalgebra $(C, \Psi)$ together with a quasi-isomorphism $\theta: \widetilde{\Omega}(C, \Psi) \stackrel{\widetilde{ }}{\rightarrow} H$ of chain algebras that is also a map of coalgebras up to strong homotopy, where the homotopies are appropriately compatible with the multiplicative structure (cf, Definition 5.1). As illustrated by the results in this article, Alexander-Whitney models can be useful tools for homology calculations in $\mathbf{H}$.

The topologist's motivation for considering the category $\mathbf{F}$ is the existence of a natural Alexander-Whitney model of the chain Hopf algebra $C(G K)$, where $K$ is a reduced simplicial set. As shown in [8], there is a functor $\widetilde{C}: \mathbf{s S e t}_{0} \rightarrow \mathbf{F}$ from the category of reduced simplicial sets to the category of Alexander-Whitney coalgebras such that for all simplicial sets $K$, there is a natural quasi-isomorphism of chain algebras

$$
\theta_{K}: \widetilde{\Omega} \widetilde{C}(K) \stackrel{\simeq}{\rightarrow} C(G K),
$$


which is also a map of coalgebras up to strong homotopy.

Given the existence of natural Alexander-Whitney models, the most important steps on the path to constructing the model $\mathfrak{L F}(f)$ and to proving that its homology algebra is isomorphic to $\mathrm{H}_{*}(G F)$ are the following.

(1) For any chain Hopf algebra $H$ and any right $H$-comodule algebra $B$, we observe that $\operatorname{Cotor}^{H}(B, R)$ admits a natural graded algebra structure (Corollary 3.8 and, more generally, Proposition 3.19). In particular, for any morphism $\varphi: H^{\prime} \rightarrow H$ of chain Hopf algebras, $\operatorname{Cotor}^{H}\left(H^{\prime}, R\right)$, which can be seen as the homology of the "homotopy fiber" of $\varphi$, admits a natural graded algebra structure.

(2) We show that the category $\mathbf{F}$ admits a natural "based-path" construction, ie a functor $\widetilde{\mathfrak{P}}: \mathbf{F} \rightarrow \mathbf{F}$ such that $\widetilde{\mathfrak{P}}(C, \Psi)$ is acyclic for all $(C, \Psi)$, together with a natural "projection" morphism in $\mathbf{F}$ from $\widetilde{\mathfrak{P}}(C, \Psi)$ to $(C, \Psi)$ (Definition 4.4).

(3) For any morphism $\omega$ : $\left(C^{\prime}, \Psi^{\prime}\right) \rightarrow(C, \Psi)$ in $\mathbf{F}$, we prove that the chain Hopf algebra

$$
\widetilde{\Omega}\left(\left(C^{\prime}, \Psi^{\prime}\right) \bigsqcup \widetilde{\mathfrak{P}}(C, \Psi)\right)
$$

is cofree over $\widetilde{\Omega}(C, \Psi)$ on a cobasis that is itself a sub chain algebra, denoted $\mathfrak{L F}(\omega)$, of $\widetilde{\Omega}\left(\left(C^{\prime}, \Psi^{\prime}\right) \amalg \widetilde{\mathfrak{P}}(C, \Psi)\right)$ (Corollary 4.7).

(4) Given an Alexander-Whitney model $\omega:\left(C^{\prime}, \Psi^{\prime}\right) \rightarrow(C, \Psi)$ of a morphism of chain Hopf algebras $\varphi: H^{\prime} \rightarrow H$, we prove that $\mathrm{H}_{*}(\mathfrak{L F}(\omega)) \cong \operatorname{Cotor}^{H}\left(H^{\prime}, R\right)$ as graded algebras (Theorem 5.6).

Let $f: K \rightarrow L$ be a simplicial morphism of 2-reduced simplicial sets with homotopy fiber $F$. Applying (4) to $\widetilde{C}(f): \widetilde{C}(K) \rightarrow \widetilde{C}(L)$, we obtain an isomorphism of algebras

$$
\mathrm{H}_{*}(G F) \cong \mathrm{H}_{*}(\mathfrak{L} \mathfrak{F}(\widetilde{C}(f))),
$$

thanks to the algebra isomorphism

$$
\mathrm{H}_{*}(G F) \cong \operatorname{Cotor}^{C(G L)}(C(G K), R)
$$

that follows from the dual of [4, Theorem 5.1].

To make this article as self-contained as possible and to establish our notation, we begin in Section 1 by recalling the rather extensive foundations on which our current research is built. Section 1.3, in which we describe the operadic approach to stronglyhomotopy coalgebra structures of Hess-Parent-Scott [6], is particularly important for the later sections of this paper and essential to providing a clean description of the 
yoga of Alexander-Whitney coalgebras. Readers unfamiliar with the role of co-rings in monoidal categories as parametrizing objects for enlarged morphism sets or with operads will find all of the necessary definitions in Section 1.1 and Section 1.2.

Section 3 concerns the naturality of multiplicative structure on Cotor, which plays an important role in the proofs of Theorem 5.4 and Theorem 5.6, the key elements of step (4) in the plan outlined above. Given chain Hopf algebras $H$ and $H^{\prime}$, as well as a right $H$-comodule algebra $B$ and a right $H^{\prime}$-comodule algebra $B^{\prime}$, there is an obvious notion of "morphisms" from $(H ; B)$ to $\left(H^{\prime} ; B^{\prime}\right)$ : the set of pairs $(f ; g)$, where $f: H \rightarrow H^{\prime}$ is a chain Hopf algebra map and $g: B \rightarrow B^{\prime}$ is a chain algebra map respecting the coactions of $H$ and $H^{\prime}$. It is easy to see that any such pair induces an algebra map $\operatorname{Cotor}^{H}(B, R) \rightarrow \operatorname{Cotor}^{H^{\prime}}\left(B^{\prime}, R\right)$. There is however a more general type of "morphism" from $(H ; B)$ to $\left(H^{\prime} ; B^{\prime}\right)$, which we call a comodule algebra map up to strong homotopy (CASH map), that also induces an algebra map $\operatorname{Cotor}^{H}(B, R) \rightarrow \operatorname{Cotor}^{H^{\prime}}\left(B^{\prime}, R\right)$.

In Section 3.2 we define CASH maps and establish existence results (Proposition 3.16 and Proposition 3.18) that we use afterwards to prove Theorem 5.4 and Theorem 5.6. Before verifying the existence results, we provide an equivalent, operadic definition of CASH maps, modeled on the operadic approach to strongly homotopy coalgebra structures, that facilitates considerably the bookkeeping involved in working with the infinite family of homotopies associated to a CASH map. The general study of monoidal structures and parametrizations by co-rings in Section 2 is essential to the development of this operadic approach.

Section 4 and Section 5 are devoted to the study of Alexander-Whitney coalgebras and their use in calculations of the homology of homotopy fibers in the category $\mathbf{H}$ of chain Hopf algebras. Topology comes into play again in Section 6, where we apply the purely algebraic results of the preceeding sections to constructing our loop-homotopy fiber model. In particular, we study the special cases of double suspensions and of formal spaces, obtaining a simplified model for the homology of their double loop spaces, which is a free algebra on a set of generators we describe completely.

The first author would like to thank the University of Aberdeen for its kind hospitality during the initial phase of research on this project, while the second author would like to thank the EPFL for hosting him during the completion of the project. Both authors would like to thank referees for pointing out the relevance of [4] to their work and for providing helpful organizational advice.

\section{Notation and conventions}

- Given objects $A$ and $B$ of a category $\mathbf{C}$, we let $\mathbf{C}(A, B)$ denote the set of morphisms with source $A$ and target $B$. 
- Throughout this paper we are working over a principal ideal domain $R$. We denote the category of graded $R$-modules by $\operatorname{grMod}_{R}$ and the category of chain complexes over $R$ by $\mathbf{C h}_{R}$. The underlying graded modules of all chain (co)algebras are assumed to be $R$-free.

- The degree of an element $v$ of a graded module $V$ is denoted either $|v|$ or simply $v$, when used as an exponent, and no confusion can arise.

- Throughout this article we apply the Koszul sign convention for commuting elements of a graded module or for commuting a morphism of graded modules past an element of the source module. For example, if $V$ and $W$ are graded algebras and $v \otimes w, v^{\prime} \otimes w^{\prime} \in V \otimes W$, then

$$
(v \otimes w) \cdot\left(v^{\prime} \otimes w^{\prime}\right)=(-1)^{|w| \cdot\left|v^{\prime}\right|} v v^{\prime} \otimes w w^{\prime} .
$$

Futhermore, if $f: V \rightarrow V^{\prime}$ and $g: W \rightarrow W^{\prime}$ are morphisms of graded modules, then for all $v \otimes w \in V \otimes W$,

$$
(f \otimes g)(v \otimes w)=(-1)^{|g| \cdot|v|} f(v) \otimes g(w) .
$$

- A graded module $V$ is bounded below if there is some $N \in \mathbb{Z}$ such that $V_{k}=0$ for all $k<N$. It is $n$-connected if, in particular, $V_{k}=0$ for all $k \leq n$. We write $V_{+}$for $V_{>0}$.

- The suspension endofunctor $s$ on the category of graded modules is defined on objects $V=\bigoplus_{i \in \mathbb{Z}} V_{i}$ by $(s V)_{i} \cong V_{i-1}$. Given a homogeneous element $v$ in $V$, we write $s v$ for the corresponding element of $s V$. The suspension $s$ admits an obvious inverse, which we denote $s^{-1}$.

- Given chain complexes $(V, d)$ and $(W, d)$, the notation $f:(V, d) \stackrel{\simeq}{\rightarrow}(W, d)$ indicates that $f$ induces an isomorphism in homology. In this case we refer to $f$ as a quasi-isomorphism.

- Let $V$ be a positively-graded $R$-module. The free associative algebra on $V$ is denoted $T V$, ie,

$$
T V \cong R \oplus V \oplus(V \otimes V) \oplus(V \otimes V \otimes V) \oplus \cdots
$$

A typical basis element of $T V$ is denoted $v_{1} \cdots v_{n}$.

- Given a comodule $(M, v)$ over a coalgebra $(C, \Delta)$, we let $\Delta^{(i-1)}$ denote the iterated comultiplication $C \rightarrow C^{\otimes i}$ and $\nu^{(i)}$ the iterated coaction $M \rightarrow M \otimes$ $C^{\otimes i}$. The reduced comultiplication is denoted $\bar{\Delta}$.

- If $C$ is a simply connected chain coalgebra with reduced comultiplication $\bar{\Delta}$ and differential $d$, then $\Omega C$ denotes the cobar construction on $C$, ie, the chain algebra $\left(T s^{-1}\left(C_{+}\right), d_{\Omega}\right)$, where $d_{\Omega}=-s^{-1} d s+\left(s^{-1} \otimes s^{-1}\right) \bar{\Delta} s$ on generators. 
Furthermore, for every pair of simply-connected chain coalgebras $C$ and $C^{\prime}$

$$
q: \Omega\left(C \otimes C^{\prime}\right) \stackrel{\simeq}{\rightarrow} \Omega C \otimes \Omega C^{\prime}
$$

denotes the quasi-isomorphism of chain algebras defined by Milgram in [11].

\section{Preliminaries}

For the convenience of the reader, we recall here certain algebraic foundations of our work. We begin by reminding the reader how co-rings in monoidal categories can act as parametrizing objects for categories of modules with enlarged morphism sets, as described in Hess-Parent-Scott [6] and Hess-Parent-Scott-Tonks [8]. We then review the theory of operads, seen as monoids with respect to a certain nonsymmetric monoidal structure on the category of symmetric sequences of objects in a given symmetric monoidal category. In particular we analyze the category of right modules over a given operad $\mathcal{P}$, comparing it to the category of $\mathcal{P}$-coalgebras. Finally, we summarize briefly results in [6] and [8] that provide an operadic description of the category DCSH of chain coalgebras and of strongly homotopy coalgebra maps, in terms of a certain co-ring over the associative operad.

\subsection{Co-rings in monoidal categories}

Let $(\mathbf{C}, \otimes, I)$ be a monoidal category, and let $(A, \mu, \eta)$ be a monoid in $\mathbf{C}$. If the category $\mathbf{C}$ admits coequalizers and $M \otimes-$ and $-\otimes N$ preserve colimits for all objects $M$ and $N$, then the category of $A$-bimodules, ${ }_{A} \mathbf{M o d}_{A}$, is a monoidal category also, with monoidal product $-\otimes_{A}-$. If $M$ and $M^{\prime}$ are $A$-bimodules, then $M \otimes_{A} M^{\prime}$ is the coequalizer of the diagram

$$
M \otimes A \otimes M^{\prime} \underset{\rho \otimes \operatorname{Id}_{M^{\prime}}}{\stackrel{\operatorname{Id}_{M} \otimes \lambda^{\prime}}{\rightrightarrows}} M \otimes M^{\prime}
$$

The unit object with respect to $-\otimes_{A}-$ is $A$ itself, where the right and left $A$-actions on $A$ are given by the multiplication map $\mu$.

Definition 1.1 An $A$-co-ring is a comonoid in the monoidal category $\left({ }_{A} \operatorname{Mod}_{A}, \otimes_{A}, A\right)$. An $A$-co-ring thus consists of an $A$-bimodule $M$, together with two morphisms of $A$-bimodules

$$
\psi: M \rightarrow M \otimes_{A} M \quad \text { and } \quad \varepsilon: M \rightarrow A
$$

such that $\psi$ is coassociative and counital with respect to $\varepsilon$. 
Examples of co-rings abound in algebra and topology. In particular, any Frobenius algebra is a co-ring over itself, while the Hopf algebroids of stable homotopy theory are co-rings with extra structure. Moreover, any ring homomorphism $\varphi: A \rightarrow B$ induces a canonical $B$-co-ring structure on $M=B \otimes_{A} B$, where the comultiplication is

$$
M \rightarrow M \otimes_{B} M: b \otimes b^{\prime} \mapsto(b \otimes 1) \otimes\left(1 \otimes b^{\prime}\right) .
$$

Co-rings play an important role in this article, as they induce natural enlargements of categories of modules, leaving the objects fixed and expanding the morphism sets. Allowing larger morphism sets translates into weakening the notion of morphism of modules. In this sense a co-ring plays the role of a family of parameters, with respect to which such a weaker notion is coherently defined.

Definition 1.2 Let $\operatorname{Mod}_{A}$ denote the category of right $A$-modules. Given an $A-$ co-ring $(M, \psi, \varepsilon)$, let $\operatorname{Mod}_{A, M}$ denote the category with $\operatorname{Ob}_{\operatorname{Mod}}, M=\operatorname{Ob}_{\operatorname{Mod}}$ and

$$
\operatorname{Mod}_{A, M}\left(N, N^{\prime}\right):=\operatorname{Mod}_{A}\left(N \otimes_{A} M, N^{\prime}\right) .
$$

Given $f \in \operatorname{Mod}_{A, M}\left(N, N^{\prime}\right)$ and $f^{\prime} \in \operatorname{Mod}_{A, M}\left(N^{\prime}, N^{\prime \prime}\right)$, their composite $f^{\prime} f \in$ $\operatorname{Mod}_{A, M}\left(N, N^{\prime \prime}\right)$ is equal to the composite in $\operatorname{Mod}_{A}$ of the following sequence of morphisms of right $A$-modules.

$$
N \otimes_{A} M \stackrel{\mathrm{Id}_{N} \otimes_{A} \psi}{\longrightarrow} N \otimes_{A} M \otimes_{A} M \stackrel{f \otimes_{A} \mathrm{Id}_{M}}{\longrightarrow} N^{\prime} \otimes_{A} M \stackrel{f^{\prime}}{\longrightarrow} N^{\prime \prime} .
$$

Composition in $\operatorname{Mod}_{A, M}$ is associative and unital, since $\psi$ is coassociative and counital. Furthermore, there is a natural, faithful functor

$$
\mathfrak{I}_{M}: \operatorname{Mod}_{A} \rightarrow \operatorname{Mod}_{A, M},
$$

which is the identity on objects and which sends a morphism $f: N \rightarrow N^{\prime}$ of right $A$-modules to

$$
\mathfrak{I}_{M}(f)=f \otimes_{A} \varepsilon: N \otimes_{A} M \rightarrow N^{\prime} \otimes_{A} A \cong N^{\prime} .
$$

The category $\operatorname{Mod}_{A, M}$ is therefore truly an enlargement of $\operatorname{Mod}_{A}$.

We conclude this section by clarifying our vision of a co-ring as a family of a parameters.

Definition 1.3 Let $(M, \psi, \varepsilon)$ be an $A$-co-ring, endowed with a strict morphism of left $A$-modules $\eta: A \rightarrow M$. Let $N, N^{\prime} \in \operatorname{Ob}_{\operatorname{Mod}}$. A morphism $f \in \mathbf{C}\left(N, N^{\prime}\right)$ is a 
morphism of right $A$-modules up to $M$-parametrization if there is a strict morphism of right $A$-modules $g: N \otimes_{A} M \rightarrow N^{\prime}$ such that the following diagram in $\mathbf{C}$ commutes.

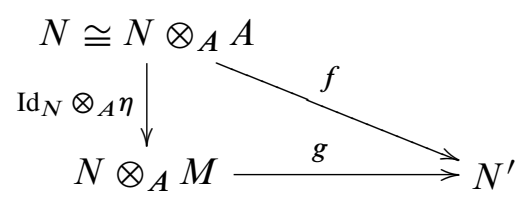

There is an analogous enlargement of the category of left $A$-modules. For the experts, we note that these enlargements are, of course, coKleisli constructions, induced by the comonads $-\otimes_{A} M$ and $M \otimes_{A}-$.

\subsection{Operads and their modules and coalgebras}

Let $(\mathbf{C}, \otimes, I)$ be a symmetric monoidal category such that $\mathbf{C}$ admits coequalizers and countable coproducts and has an initial object 0 . Let $\mathbf{C}^{\Sigma}$ denote the category of symmetric sequences in $\mathbf{C}$. An object $\mathcal{X}$ of $\mathbf{C}^{\Sigma}$ is a family $\{\mathcal{X}(n) \in \mathbf{C} \mid n \geq 0\}$ of objects in $\mathbf{C}$ such that $\mathcal{X}(n)$ admits a right action of the symmetric group $\Sigma_{n}$, for all $n$. The object $\mathcal{X}(n)$ is called the $n$th level of the symmetric sequence $\mathcal{X}$.

For all $\mathcal{X}, \mathcal{Y} \in \mathbf{C}^{\Sigma}$, a morphism of symmetric sequences $\varphi: \mathcal{X} \rightarrow \mathcal{Y}$ consists of a family

$$
\left\{\varphi_{n} \in \mathbf{C}(\mathcal{X}(n), \mathcal{Y}(n)) \mid \varphi_{n} \text { is } \Sigma_{n} \text {-equivariant, } n \geq 0\right\} .
$$

More formally, $\mathbf{C}^{\Sigma}$ is the category of contravariant functors from the symmetric groupoid $\boldsymbol{\Sigma}$ to $\mathbf{C}$, where $\mathrm{Ob} \boldsymbol{\Sigma}=\mathbb{N}$, the set of natural numbers, and $\boldsymbol{\Sigma}(m, n)$ is empty if $m \neq n$, while $\Sigma(n, n)=\Sigma_{n}$.

The category $\mathbf{C}$ can be "linearly" embedded in the category $\mathbf{C}^{\Sigma}$, via a functor

$$
\mathcal{L}: \mathbf{C} \rightarrow \mathbf{C}^{\Sigma},
$$

which is defined on $A \in \mathrm{Ob} \mathbf{C}$ by $\mathcal{L}(A)(1)=A$ and $\mathcal{L}(A)(n)=0$ for all $n \neq 1$ and similarly for morphisms.

There is another important embedding of $\mathbf{C}$ into $\mathbf{C}^{\Sigma}$

$$
\mathcal{T}: \mathbf{C} \rightarrow \mathbf{C}^{\Sigma}
$$

defined by $\mathcal{T}(A)(n)=A^{\otimes n}$ for all $n$. The right action of $\Sigma_{n}$ on $\mathcal{T}(A)(n)=A^{\otimes n}$ is given by permutation of the factors, using iterates of the natural symmetry isomorphism $\tau: A \otimes A \stackrel{\cong}{\rightrightarrows} A \otimes A$ in $\mathbf{C}$. For example, if $\mathbf{C}$ is the category of graded modules, then

$$
\left(a_{1} \otimes \cdots \otimes a_{n}\right) \cdot \sigma=a_{\sigma(1)} \otimes \cdots \otimes a_{\sigma(n)}
$$


for all $a_{1}, \ldots, a_{n} \in A$.

As a first indication of the role of differential structure in symmetric sequences, we introduce the following useful operation on symmetric sequences of chain complexes in the image of $\mathcal{T}$. The analogy with the notion of a derivation on an algebra is evident.

Definition 1.4 Let $f, g, t: A \rightarrow B$ be morphisms of graded $R$-modules, homogeneous of degrees 0,0 and $m$, respectively. The $(f, g)$-derivation of symmetric sequences induced by $t$ is the morphism of symmetric sequences

$$
\mathcal{D}_{(f, g)}(t): \mathcal{T}(A) \rightarrow \mathcal{T}(B)
$$

that is of degree $m$ in each level and that is defined as follows in level $n$.

$$
\mathcal{D}_{(f, g)}(t)_{n}=\sum_{j=0}^{n-1} f^{\otimes j} \otimes t \otimes g^{\otimes n-j-1} .
$$

When $A=B$ and $f=\operatorname{Id}_{A}=g$, we simplify notation and write $\mathcal{D}(t)$ for the $\left(\operatorname{Id}_{A}, \operatorname{Id}_{A}\right)-$ derivation induced by $t$.

Example 1.5 If $C$ is chain complex with differential $d$, then the levelwise differential on $\mathcal{T}(C)$ is $\mathcal{D}(d)$.

In this article we use the following two monoidal structures on the category of symmetric sequences.

Definition 1.6 The level tensor product of two symmetric sequences $\mathcal{X}$ and $\mathcal{Y}$ is the symmetric sequence given by

$$
(\mathcal{X} \otimes \mathcal{Y})(n)=\mathcal{X}(n) \otimes \mathcal{Y}(n) \quad(n \geq 0),
$$

endowed with the diagonal action of $\Sigma_{n}$.

The following, well-known result is very easy to prove.

Proposition 1.7 Let $\mathcal{C}=\{\mathcal{C}(n)\}_{n \geq 0}$ be the symmetric sequence with $\mathcal{C}(n)=I$ and trivial $\Sigma_{n}$-action, for all $n \geq 0$. Then $\left(\mathbf{C}^{\Sigma}, \otimes, \mathcal{C}\right)$ is a closed symmetric monoidal category, called the level monoidal structure on $\mathbf{C}^{\Sigma}$.

A (co)monoid in $\mathbf{C}^{\Sigma}$ with respect to the level monoidal structure is called a level (co)monoid.

Note that the functor $\mathcal{T}$ is strong monoidal with respect to the level monoidal structure on symmetric sequences, ie, for all $C, C \in \mathrm{Ob} \mathbf{C}$, there is a natural isomorphism 
$\mathcal{T}\left(C \otimes C^{\prime}\right) \cong \mathcal{T}(C) \otimes \mathcal{T}\left(C^{\prime}\right)$, given in each level by iterated application of the natural symmetry isomorphism in $\mathbf{C}$.

The category $\mathbf{C}^{\Sigma}$ also admits a nonsymmetric, right-closed monoidal structure, defined as follows.

Definition 1.8 The composition tensor product of two symmetric sequences $\mathcal{X}$ and $\mathcal{Y}$ is the symmetric sequence $\mathcal{X} \diamond \mathcal{Y}$ given by

$$
(\mathcal{X} \diamond \mathcal{Y})(n)=\coprod_{\substack{k \geq 1 \\ \vec{n} \in I_{k, n}}} \mathcal{X}(k) \otimes_{\Sigma_{k}}\left(\mathcal{Y}\left(n_{1}\right) \otimes \cdots \otimes \mathcal{Y}\left(n_{k}\right)\right) \otimes \Sigma_{\vec{n}} I\left[\Sigma_{n}\right],
$$

where $I_{k, n}=\left\{\vec{\imath}=\left(n_{1}, \ldots, n_{k}\right) \in \mathbb{N}^{k} \mid \sum_{j} n_{j}=n\right\}$ and $\Sigma_{\vec{n}}=\Sigma_{n_{1}} \times \cdots \times \Sigma_{n_{k}}$, seen as a subgroup of $\Sigma_{n}$. The left action of $\Sigma_{k}$ on $\bigsqcup_{\vec{n} \in I_{k, n}} \mathcal{Y}\left(n_{1}\right) \otimes \cdots \otimes \mathcal{Y}\left(n_{k}\right)$ is given by permutation of the factors, using the natural symmetry isomorphism $A \otimes B \cong B \otimes A$ in $\mathbf{C}$.

Proposition 1.9 Let $\mathcal{J}$ denote the symmetric sequence with $\mathcal{J}(1)=1$ and $\mathcal{J}(n)=0$ otherwise, with trivial $\Sigma_{n}$-action. Then $\left(\mathbf{C}^{\Sigma}, \diamond, \mathcal{J}\right)$ is a right-closed monoidal category, called the composition monoidal structure on $\mathbf{C}^{\Sigma}$.

A proof of this result can be found in Markl-Shnider-Stasheff [10, section II.1.8].

Unwrapping the definition of the composition product of symmetric sequences, we obtain the next, well-known lemma, which tells us which data determine a morphism with source a composition of symmetric sequences.

Lemma 1.10 (Markl [9]) Let $\mathcal{X}, \mathcal{Y}$ and $\mathcal{Z}$ be symmetric sequences in $\mathbf{C}$. Let $I_{m, n}=\left\{\vec{n}=\left(n_{1}, \ldots, n_{m}\right) \mid \sum_{j} n_{j}=n\right\}$. Let

$$
\mathfrak{F}=\left\{\mathcal{X}(m) \otimes\left(\mathcal{Y}\left(n_{1}\right) \otimes \cdots \otimes \mathcal{Y}\left(n_{m}\right)\right) \stackrel{\theta_{\vec{n}}}{\longrightarrow} \mathcal{Z}(n) \mid n \geq 0, m \geq 1, \vec{n} \in I_{m, n}\right\}
$$

be a family of morphisms in C. If the following diagrams commute for all $m, n$, $\vec{n}, \sigma \in \Sigma_{m}$ and $\tau_{j} \in \Sigma_{n_{j}}$ for $1 \leq j \leq m$, then $\mathfrak{F}$ induces a morphism of symmetric sequences $\theta: \mathcal{X} \diamond \mathcal{Y} \rightarrow \mathcal{Z}$.

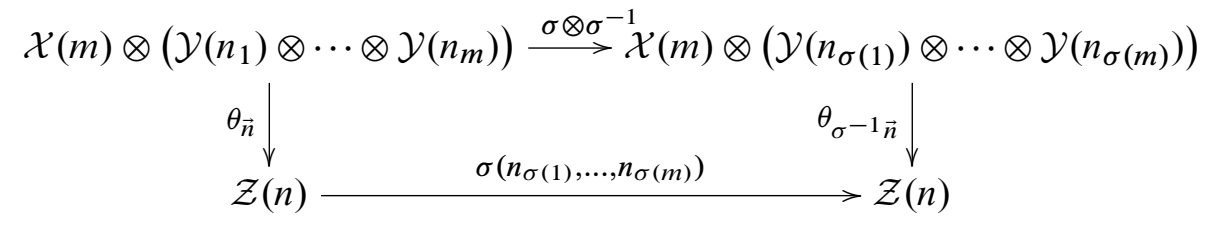

Algebraic 83 Geometric Topology, Volume 7 (2007) 


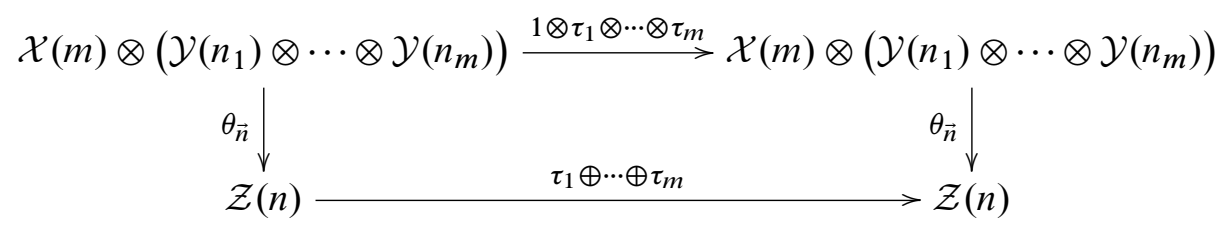

In the statement above, $\sigma^{-1} \vec{n}:=\left(n_{\sigma(1)}, \ldots, n_{\sigma(m)}\right)$, which defines a left action of $\Sigma_{m}$ on $I_{m, n}$.

Remark 1.11 For any objects $\mathcal{X}, \mathcal{X}^{\prime}, \mathcal{Y}, \mathcal{Y}^{\prime}$ in $\mathbf{C}^{\Sigma}$, there is an obvious, natural intertwining map

$$
\mathfrak{i}:\left(\mathcal{X} \otimes \mathcal{X}^{\prime}\right) \diamond\left(\mathcal{Y} \otimes \mathcal{Y}^{\prime}\right) \longrightarrow(\mathcal{X} \diamond \mathcal{Y}) \otimes\left(\mathcal{X}^{\prime} \diamond \mathcal{Y}^{\prime}\right)
$$

Definition 1.12 An operad in $\mathbf{C}$ is a monoid with respect to the composition product, ie, a triple $(\mathcal{P}, \gamma, \eta)$, where $\gamma: \mathcal{P} \diamond \mathcal{P} \rightarrow \mathcal{P}$ and $\eta: \mathcal{J} \rightarrow \mathcal{P}$ are morphisms in $\mathbf{C}^{\Sigma}$, and $\gamma$ is appropriately associative and unital with respect to $\eta$. A morphism of operads is a monoid morphism in the category of symmetric sequences.

The most important example of an operad in this paper is the associative operad $\mathcal{A}$, given by $\mathcal{A}(n)=I\left[\Sigma_{n}\right]$ for all $n$, endowed with the obvious multiplication map, induced by permutation of blocks.

Operads derive their importance from their role in parametrizing $n$-ary (co)operations and governing the identites among them. In this article we focus on cooperations and thus on coalgebras over an operad $\mathcal{P}$. A $\mathcal{P}$-coalgebra is an object $A$ of $\mathbf{C}$ along with a sequence of structure morphisms

$$
\theta_{n}: A \otimes \mathcal{P}(n) \rightarrow A^{\otimes n}, \quad n \geq 0
$$

that are appropriately associative, equivariant, and unital. We refer the reader to eg, [10], for the full definition.

A morphism of $\mathcal{P}$-coalgebras is a morphism in $\mathbf{C}$ that commutes with the coalgebra structure maps. The category of $\mathcal{P}$-coalgebras and their morphisms is denoted $\mathcal{P}$-Coalg.

Remark 1.13 Algebraists are used to thinking of coalgebras as modules with additional structure. It is important to note that if $\mathcal{P}$ is an operad, then a $\mathcal{P}$-module (in the sense defined in Section 1.1) is a object of $\mathbf{C}^{\Sigma}$ with additional structure, while a $\mathcal{P}$-coalgebra is an object of $\mathbf{C}$ with additional structure. 
On the other hand, as explained in [6, Section 2.2], the functor $\mathcal{T}$ restricts to a faithful functor

$$
\mathcal{T}: \mathcal{P}-\text { Coalg } \longrightarrow \operatorname{Mod}_{\mathcal{P}}
$$

from the category of $\mathcal{P}$-coalgebras to the category of right $\mathcal{P}$-modules (with respect to the composition product $\diamond)$, ie $\mathcal{P}$-coalgebra structure on an object $A$ in $\mathbf{C}$ induces a right $\mathcal{P}$-action map $\mathcal{T}(A) \diamond \mathcal{P} \rightarrow \mathcal{T}(A)$ in $\mathbf{C}^{\Sigma}$.

Let $(\mathcal{M}, \psi, \varepsilon)$ be a $\mathcal{P}$-co-ring, and consider $\operatorname{Mod}_{\mathcal{P}, \mathcal{M}}$, the enlarged version of $\operatorname{Mod}_{\mathcal{P}}$ described in the Section 1.1. Define an enlarged version $(\mathcal{P}, \mathcal{M})-$ Coalg of $\mathcal{P}$-Coalg by $\operatorname{Ob}(\mathcal{P}, \mathcal{M})-$ Coalg $=\mathrm{Ob} \mathcal{P}-$ Coalg

$(1-4)$

$$
(\mathcal{P}, \mathcal{M})-\operatorname{Coalg}\left(A, A^{\prime}\right):=\operatorname{Mod}_{\mathcal{P}, \mathcal{M}}\left(\mathcal{T}(A), \mathcal{T}\left(A^{\prime}\right)\right)=\operatorname{Mod}_{\mathcal{P}}\left(\mathcal{T}(A) \diamond_{\mathcal{P}} \mathcal{M}, \mathcal{T}\left(A^{\prime}\right)\right),
$$

for all $A, A^{\prime} \in \operatorname{Ob}(\mathcal{P}, \mathcal{M})-$ Coalg, with composition defined as in $\operatorname{Mod}_{\mathcal{P}, \mathcal{M}}$.

Let $A$ and $A^{\prime}$ be $\mathcal{P}$-coalgebras. In keeping with Definition 1.3, we say that a morphism $f: A \rightarrow A^{\prime}$ is a morphism of $\mathcal{P}$-coalgebras up to $\mathcal{M}$-parametrization if $\mathcal{T}(f): \mathcal{T}(A) \rightarrow \mathcal{T}\left(A^{\prime}\right)$ is a morphism of right $\mathcal{P}$-modules up to $\mathcal{M}$-parametrization.

From this formulation, it follows that co-rings over operads are, in a strong sense, relative operads. They parametrize higher, "up to homotopy" structure on morphisms of $\mathcal{P}$-coalgebras and govern relations among the higher homotopies and the $n$-ary cooperations on the source and target.

\subsection{Strongly-homotopy coalgebra structures}

The category DCSH of coassociative chain coalgebras and of coalgebra morphisms up to strong homotopy was first defined by Gugenheim and Munkholm in the early 1970s [5], when they were studying extended naturality of the functor Cotor. Its objects are simply connected, augmented, coassociative chain coalgebras, and a morphism from $C$ to $C^{\prime}$ is a map of chain algebras $\Omega C \rightarrow \Omega C^{\prime}$. The category DCSH plays an important role in topology (cf, Theorem 2.13).

In a slight abuse of terminology, we say that a chain map between chain coalgebras $f: C \rightarrow C^{\prime}$ is a DCSH map if there is a morphism in $\operatorname{DCSH}\left(C, C^{\prime}\right)$ of which $f$ is the linear part. In other words, there is a map of chain algebras $g: \Omega C \rightarrow \Omega C^{\prime}$ such that

$$
\left.g\right|_{s^{-1} C_{+}}=s^{-1} f s+\text { higher-order terms. }
$$

Let $\mathcal{A}$ denote the associative operad in the category of chain complexes. In [6] the authors constructed an $\mathcal{A}$-co-ring $\mathcal{F}$, called the Alexander-Whitney co-ring, which 
can applied in the framework of Section 1.1 to providing an operadic description of DCSH. The co-ring $\mathcal{F}$ also admits a level comultiplication $\Delta_{\mathcal{F}}: \mathcal{F} \rightarrow \mathcal{F} \otimes \mathcal{F}$ that is compatible with its composition comultiplication $\psi_{\mathcal{F}}: \mathcal{F} \rightarrow \mathcal{F} \diamond_{\mathcal{A}} \mathcal{F}$ and that plays an important role in development of monoidal structure in DCSH (cf Section 2.2).

The symmetric sequence of graded modules underlying $\mathcal{F}$ is $\mathcal{A} \diamond \mathcal{S} \diamond \mathcal{A}$, where, for all $n \geq 1, \mathcal{S}(n)=R\left[\Sigma_{n}\right] \cdot z_{n-1}$, the free $R\left[\Sigma_{n}\right]$-module on a generator of degree $n-1$, and $\mathcal{S}(0)=0$. We refer the reader to [8, pages 853-854] for the explicit formulas for the differential $\partial_{\mathcal{F}}: \mathcal{F} \rightarrow \mathcal{F}$, the composition comultiplication $\psi_{\mathcal{F}}$ and the level comultiplication $\Delta_{\mathcal{F}}$. We remark that $\mathcal{F}$ admits a natural filtration with respect to which both $\psi_{\mathcal{F}}$ and $\Delta_{\mathcal{F}}$ are filtration-preserving, while $\partial_{\mathcal{F}}$ is filtration-decreasing.

Consider $(\mathcal{A}, \mathcal{F})$-Coalg $(\operatorname{cf}(1-4))$. Any morphism $\theta \in(\mathcal{A}, \mathcal{F})-\operatorname{Coalg}\left(C, C^{\prime}\right)$ gives rise to a family $\mathfrak{F}(\theta)$ of linear maps from $C$ into $\mathcal{T}\left(C^{\prime}\right)$, defined as follows.

$$
\mathfrak{F}(\theta):=\left\{\theta_{k}=\theta\left(-\otimes z_{k-1}\right): C \rightarrow\left(C^{\prime}\right)^{\otimes k}=\mathcal{T}\left(C^{\prime}\right)(k) \mid k \geq 1\right\} .
$$

The existence of such a family $\mathfrak{F}(\theta)$ is equivalent to the existence of a morphism of symmetric sequences of graded modules $\mathcal{L}(C) \diamond \mathcal{S} \rightarrow \mathcal{T}\left(C^{\prime}\right)$, where $\mathcal{L}: \operatorname{grMod}_{R} \rightarrow$ $\operatorname{grMod}_{R}^{\Sigma}$ is the "linear" embedding (1-2). We show below (Proposition 2.7) that, under certain conditions, the existence of such a family implies that of a corresponding map in $(\mathcal{A}, \mathcal{F})$-Coalg.

The important result below follows immediately from the Cobar Duality Theorem in [6].

Theorem 1.14 (Hess, Parent and Scott [6]) There is a full and faithful functor, called the induction functor,

$$
\text { Ind: }(\mathcal{A}, \mathcal{F})-\text { Coalg } \rightarrow \mathcal{A}-\mathbf{A l g}
$$

defined on objects by $\operatorname{Ind}(C)=\Omega C$ for all $C \in \operatorname{Ob}(\mathcal{A}, \mathcal{F})$-Coalg and on morphisms by

$$
\left.\operatorname{Ind}(\theta)\right|_{s^{-1} C}=\sum_{k \geq 1}\left(s^{-1}\right)^{\otimes k} \theta\left(-\otimes z_{k-1}\right) s: s^{-1} C_{+} \rightarrow \Omega C^{\prime}
$$

for all $\theta \in(\mathcal{A}, \mathcal{F})-\operatorname{Coalg}\left(C, C^{\prime}\right)$.

As an easy consequence of Theorem 1.14, we obtain the following crucial operadic characterization of DCSH.

Theorem 1.15 (Hess, Parent and Scott [6]) There is an isomorphism of categories

$$
(\mathcal{A}, \mathcal{F})-\text { Coalg } \stackrel{\cong}{\longrightarrow} \text { DCSH }
$$

defined to be the identity on objects and to be Ind on morphisms. 
Remark 1.16 Thanks to this operadic description of DCSH, we see that strongly homotopy coalgebra maps are exactly morphisms of $\mathcal{A}$-coalgebras up to $\mathcal{F}$-parametrization.

\section{Monoidal structures and modules over operads}

We carry out in this section a detailed study of monoidal structures on categories of modules and of coalgebras over a fixed operad $\mathcal{P}$, in both their usual and enlarged, "upto-parametrization" forms, with respect to some $\mathcal{P}$-co-ring $\mathcal{Q}$. We devote particular attention to the monoids in these categories, which we call $\mathcal{P}$-rings (in $\operatorname{Mod}_{\mathcal{P}}$ ), pseudo $\mathcal{P}$-rings (in $\mathbf{M o d}_{\mathcal{P}, \mathcal{Q}}$ ), $\mathcal{P}$-Hopf algebras (in $\mathcal{P}$-Coalg) and pseudo $\mathcal{P}$-Hopf algebras (in $(\mathcal{P}, \mathcal{Q})-$ Coalg). We begin by treating the general case, then specialize to $(\mathcal{A}, \mathcal{F})$-Coalg .

\subsection{Monoidal structures and co-ring parametrizations}

Let $(\mathbf{C}, \otimes, I)$ be any symmetric monoidal category admitting coequalizers and coproducts. Thanks to the existence and naturality of the intertwining map $\mathfrak{i}$ (Remark 1.11), the level tensor product of symmetric sequences induces a symmetric monoidal structure $\wedge$ on the category of operads. If $(\mathcal{P}, \gamma)$ and $\left(\mathcal{P}^{\prime}, \gamma^{\prime}\right)$ are operads, then $(\mathcal{P}, \gamma) \wedge\left(\mathcal{P}^{\prime}, \gamma^{\prime}\right):=\left(\mathcal{P} \otimes \mathcal{P}^{\prime}, \gamma^{\prime \prime}\right)$, where $\gamma^{\prime \prime}$ is the composite

$$
\left(\mathcal{P} \otimes \mathcal{P}^{\prime}\right) \diamond\left(\mathcal{P} \otimes \mathcal{P}^{\prime}\right) \stackrel{\mathfrak{i}}{\rightarrow}(\mathcal{P} \diamond \mathcal{P}) \otimes\left(\mathcal{P}^{\prime} \diamond \mathcal{P}^{\prime}\right) \stackrel{\gamma \otimes \gamma^{\prime}}{\longrightarrow} \mathcal{P} \otimes \mathcal{P}^{\prime} .
$$

The unit object with respect to the monoidal product $\wedge$ is $\mathcal{C}$ (cf Proposition 1.7).

Henceforth, let $(\mathcal{P}, \gamma, \Delta, \epsilon)$ be a Hopf operad, ie, a level comonoid in the category of operads: $\Delta: \mathcal{P} \rightarrow \mathcal{P} \wedge \mathcal{P}$ is a coassociative morphism of operads that is counital with respect to $e: \mathcal{P} \rightarrow \mathcal{C}$, which is also a morphism of operads. The category $\operatorname{Mod}_{\mathcal{P}}$ of right $\mathcal{P}$-modules then admits a symmetric monoidal product, also denoted $\wedge$, which is defined as follows. If $(\mathcal{M}, \rho)$ and $\left(\mathcal{M}^{\prime}, \rho^{\prime}\right)$ are two right $\mathcal{P}$-modules, then $(\mathcal{M}, \rho) \wedge\left(\mathcal{M}^{\prime}, \rho^{\prime}\right):=\left(\mathcal{M} \otimes \mathcal{M}^{\prime}, \rho^{\prime \prime}\right)$, where $\rho^{\prime \prime}$ is the composite

$\left(\mathcal{M} \otimes \mathcal{M}^{\prime}\right) \diamond \mathcal{P} \stackrel{1 \diamond \Delta}{\longrightarrow}\left(\mathcal{M} \otimes \mathcal{M}^{\prime}\right) \diamond(\mathcal{P} \otimes \mathcal{P}) \stackrel{\mathfrak{i}}{\rightarrow}(\mathcal{M} \diamond \mathcal{P}) \otimes\left(\mathcal{M}^{\prime} \diamond \mathcal{P}\right) \stackrel{\rho \otimes \rho^{\prime}}{\longrightarrow} \mathcal{M} \otimes \mathcal{M}^{\prime}$.

The unit object is $\mathcal{C}$, endowed with the right $\mathcal{P}$-action given by the composite

$$
\mathcal{C} \diamond \mathcal{P} \stackrel{1 \diamond \varepsilon}{\longrightarrow} \mathcal{C} \diamond \mathcal{C} \stackrel{\gamma_{\mathcal{C}}}{\longrightarrow} \mathcal{C},
$$

where $\gamma_{\mathcal{C}}$ is the usual multiplication on $\mathcal{C}$. 
There is an induced, symmetric monoidal structure $(\mathcal{P}-$ Coalg, $\wedge, I)$ such that there is a natural isomorphism of functors $\mathcal{T}(-\wedge-) \cong \mathcal{T}(-) \wedge \mathcal{T}(-)$ from $\mathbf{C}$ into $\operatorname{Mod}_{\mathcal{P}}$.

The category of monoids in $\left(\operatorname{Mod}_{\mathcal{P}}, \wedge, \mathcal{P}\right)$ and morphisms thereof is denoted $\mathbf{R i n g}_{\mathcal{P}}$. We call the objects of this category $\mathcal{P}$-rings. Restricting to monoids in $(\mathcal{P}-$ Coalg $\wedge, I)$, we obtain the category $\mathcal{P}-$ Hopf of $\mathcal{P}-$ Hopf algebras.

The categories ${ }_{\mathcal{P}} \operatorname{Mod}$ of left $\mathcal{P}$-modules and ${ }_{\mathcal{P}} \operatorname{Mod}_{\mathcal{P}}$ of $\mathcal{P}$-bimodules over $(\mathcal{P}, \gamma, \Delta)$ also admit symmetric, level-monoidal structures, defined analogously to that on $\operatorname{Mod}_{\mathcal{P}}$. The category of $\mathcal{P}$-bimodules is endowed with a second, nonsymmetric monoidal structure derived from the composition structure. Given two $\mathcal{P}$-bimodules $\mathcal{M}$ and $\mathcal{N}$, their composition product over $\mathcal{P}$, denoted $\mathcal{M} \diamond_{\mathcal{P}} \mathcal{N}$, is defined to be the obvious coequalizer. Naturality arguments show that the intertwining map induces a natural morphism of $\mathcal{P}$-bimodules

$$
\mathfrak{i}:\left(\mathcal{X} \wedge \mathcal{X}^{\prime}\right) \diamond_{\mathcal{P}}\left(\mathcal{Y} \wedge \mathcal{Y}^{\prime}\right) \longrightarrow\left(\mathcal{X} \diamond_{\mathcal{P}} \mathcal{Y}\right) \wedge\left(\mathcal{X}^{\prime} \diamond_{\mathcal{P}} \mathcal{Y}^{\prime}\right)
$$

intertwining $\wedge$ and $\diamond_{\mathcal{P}}$.

Definition 2.1 A level-comonoidal $\mathcal{P}$-co-ring is a $\mathcal{P}$-co-ring $\left(\mathcal{Q}, \psi_{\mathcal{Q}}, \varepsilon_{\mathcal{Q}}\right)$ endowed with a coassociative, level comultiplication

$$
\Delta_{\mathcal{Q}}: \mathcal{Q} \rightarrow \mathcal{Q} \wedge \mathcal{Q},
$$

which is counital with respect to

$$
e_{\mathcal{Q}}: \mathcal{Q} \rightarrow \mathcal{C}
$$

Furthermore, the diagrams

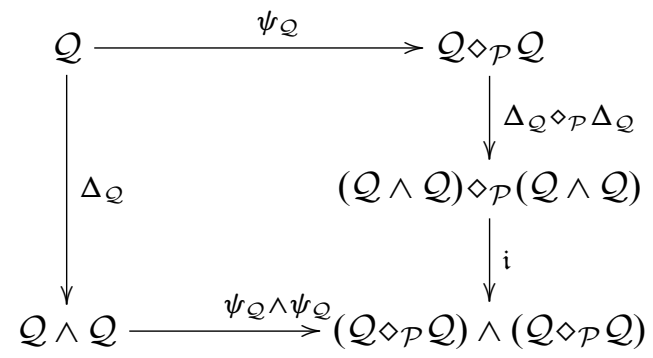

and

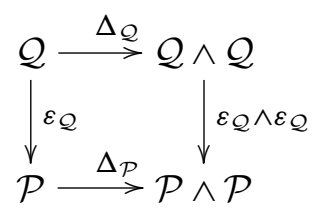

must commute. 
Recall the "inclusion" functor (1-1)

$$
\mathfrak{I}_{\mathcal{Q}}: \operatorname{Mod}_{\mathcal{P}} \rightarrow \operatorname{Mod}_{\mathcal{P}, \mathcal{Q}}
$$

Restricting $\mathfrak{I}_{\mathcal{Q}}$ to $\mathcal{P}$-Coalg defines an "inclusion" functor

$$
\mathfrak{I}_{\mathcal{Q}}: \mathcal{P}-\text { Coalg } \rightarrow(\mathcal{P}, \mathcal{Q})-\text { Coalg, }
$$

where $\Im_{\mathcal{Q}}$ is defined on a morphism $f: C \rightarrow C^{\prime}$ by $\Im_{\mathcal{Q}}(f):=\mathcal{T}(f) \diamond_{\mathcal{P}} \varepsilon_{\mathcal{Q}}$.

Bringing the level comultiplication $\Delta_{\mathcal{Q}}$ into play, we can define a symmetric monoidal product $\curlywedge$ on $\operatorname{Mod}_{\mathcal{P}, \mathcal{Q}}$ as follows, so that the restriction to $\operatorname{Mod}_{\mathcal{P}}$ is the same as $\wedge$. On objects, $\mathcal{M} \wedge \mathcal{N}$ is the same as $\mathcal{M} \wedge \mathcal{N}$ in $\operatorname{Mod}_{\mathcal{P}}$, while the monoidal product $\theta \curlywedge \theta^{\prime}$ of $\theta \in \operatorname{Mod}_{\mathcal{P}, \mathcal{Q}}(\mathcal{M}, \mathcal{N})$ and $\theta^{\prime} \in \operatorname{Mod}_{\mathcal{P}, \mathcal{Q}}\left(\mathcal{M}^{\prime}, \mathcal{N}^{\prime}\right)$ is defined to be the following composite of morphisms of strict $\mathcal{P}$-bimodules.

$\left(\mathcal{M} \wedge \mathcal{M}^{\prime}\right) \diamond_{\mathcal{P}} \mathcal{Q} \stackrel{1 \diamond_{\mathcal{P}} \Delta_{\mathcal{Q}}}{\longrightarrow}\left(\mathcal{M} \wedge \mathcal{M}^{\prime}\right) \diamond_{\mathcal{P}}(\mathcal{Q} \wedge \mathcal{Q}) \stackrel{\mathfrak{i}}{\rightarrow}\left(\mathcal{M} \diamond_{\mathcal{P}} \mathcal{Q}\right) \wedge\left(\mathcal{M}^{\prime} \diamond_{\mathcal{P}} \mathcal{Q}\right) \stackrel{\theta \wedge \theta^{\prime}}{\longrightarrow} \mathcal{M} \wedge \mathcal{M}^{\prime}$

The compatibility of the two comultiplications implies that $-\curlywedge-$ is indeed a bifunctor. The coassociativity of $\Delta_{\mathcal{Q}}$ ensures the associativity of $\curlywedge$, while the counit of $\Delta_{\mathcal{Q}}$ gives rise to the unit of $\curlywedge$. By restriction, and using that $\mathcal{T}$ is strong monoidal, we obtain a monoidal structure $\curlywedge$ on $(\mathcal{P}, \mathcal{Q})-$ Coalg, which is the usual monoidal product of $\mathcal{P}$-coalgebras on objects.

Definition 2.2 The category of monoids in $\operatorname{Mod}_{\mathcal{P}, \mathcal{Q}}$ with respect to the monoidal product $\curlywedge$ and of their morphisms is denoted $\operatorname{PsRing}_{\mathcal{P}, \mathcal{Q}}$. We call its objects pseudo $\mathcal{P}-$ rings, suppressing explicit mention of the governing comultiplication $\psi_{\mathcal{Q}}$. Restricting to $\curlywedge$-monoids in $(\mathcal{P}, \mathcal{Q})$-Coalg, we obtain the category $(\mathcal{P}, \mathcal{Q})$-PsHopf of pseudo $\mathcal{P}$-Hopf algebras.

If $(\mathcal{B}, \mu)$ is a $\mathcal{P}$-ring, where $\mu \in \operatorname{Mod}_{\mathcal{P}}(\mathcal{B} \wedge \mathcal{B}, \mathcal{B})$ is the product map, it is clear that $\left(\mathfrak{I}_{\mathcal{Q}}(\mathcal{B}), \mathfrak{I}_{\mathcal{Q}}(\mu)\right)$ is a pseudo $\mathcal{P}$-ring. In other words, $\mathfrak{I}_{\mathcal{Q}}$ induces an "inclusion" functor

$$
\mathfrak{I}_{\mathcal{Q}}: \text { Ring }_{\mathcal{P}} \rightarrow \text { PsRing }_{\mathcal{P}, \mathcal{Q}}
$$

Similarly, there is an induced, "inclusion" functor

$$
\mathfrak{I}_{\mathcal{Q}}: \mathcal{P}-\text { Hopf } \rightarrow(\mathcal{P}, \mathcal{Q}) \text {-PsHopf. }
$$

When the $\mathcal{P}$-bimodule $\mathcal{Q}$ is a free bimodule, there exist "free" constructions in the category of pseudo $\mathcal{P}$-Hopf algebras, as specified in the next proposition. Before stating the proposition, we state and prove a crucial lemma, which is useful elsewhere in this article as well, then introduce one necessary definition. 
Restricting to $\mathbf{C}=\operatorname{grMod}_{R}$ or $\mathbf{C}=\mathbf{C h}_{R}$, let $\mathcal{L}: \mathbf{C} \rightarrow \mathbf{C}^{\Sigma}$ be the "linear" embedding of (1-2). Let $\mathfrak{u}: \mathcal{L} \rightarrow \mathcal{T}$ denote the obvious "inclusion on level 1" natural transformation.

Lemma 2.3 Let $A$ and $B$ be graded $R$-modules, and let $\mathcal{X}$ be a symmetric sequence of graded $R$-modules. Any morphism $\theta: \mathcal{L}(A) \diamond \mathcal{X} \rightarrow \mathcal{T}(B)$ in $\operatorname{grMod}_{R}^{\Sigma}$ gives rise naturally to a morphism $\widehat{\theta}: \mathcal{T}(A) \diamond \mathcal{X} \rightarrow \mathcal{T}(B)$ of symmetric sequences such that $\widehat{\theta}(\mathfrak{u} \diamond \mathrm{Id})=\theta$.

Proof Recall from Definition 1.8 that, in the definition of the composition product of symmetric sequences $\mathcal{X}$ and $\mathcal{Y}$, the left action of $\Sigma_{k}$ on $\coprod_{\vec{n} \in I_{k, n}} \mathcal{Y}\left(n_{1}\right) \otimes \cdots \otimes \mathcal{Y}\left(n_{k}\right)$
is given by

$$
\sigma \cdot\left(y_{1} \otimes \cdots \otimes y_{k}\right)=y_{\sigma^{-1}(1)} \otimes \cdots \otimes y_{\sigma^{-1}(k)}
$$

for all $\sigma \in \Sigma_{k}$ and $y_{i} \in \mathcal{Y}\left(n_{i}\right), 1 \leq i \leq k$.

For all $m, n$ and $\vec{n} \in I_{m, n}$, define

$$
\widehat{\theta}_{\vec{n}}: \mathcal{T}(A)(m) \otimes\left(\mathcal{Y}\left(n_{1}\right) \otimes \cdots \otimes \mathcal{Y}\left(n_{m}\right)\right) \longrightarrow \mathcal{T}(B)(n)
$$

to be the composite

$$
\begin{gathered}
A^{\otimes m} \otimes\left(\mathcal{Y}\left(n_{1}\right) \otimes \cdots \otimes \mathcal{Y}\left(n_{m}\right)\right) \stackrel{\cong}{\cong}\left(A \otimes \mathcal{Y}\left(n_{1}\right)\right) \otimes \cdots \otimes\left(A \otimes \mathcal{Y}\left(n_{m}\right)\right) \\
\theta^{\otimes m} \downarrow \\
B^{\otimes n_{1}} \otimes \cdots \otimes B^{\otimes n_{m}} \\
=\downarrow \begin{array}{l}
\downarrow \\
B^{\otimes n} .
\end{array}
\end{gathered}
$$

Since $\theta$ is a morphism of symmetric sequences, the second diagram in Lemma 1.10 commutes for $\mathcal{X}=\mathcal{T}(A)$ and $\mathcal{Z}=\mathcal{T}(B)$. The first diagram commutes in this case as well because for all $a_{1} \otimes \cdots \otimes a_{m} \in A^{\otimes m}$ and all $y_{1} \otimes \cdots \otimes y_{m} \in \mathcal{Y}\left(n_{1}\right) \otimes \cdots \otimes \mathcal{Y}\left(n_{m}\right)$,

$$
\begin{aligned}
& \widehat{\theta}_{\sigma^{-1} \vec{n}}\left(\sigma \otimes \sigma^{-1}\right)\left(\left(a_{1} \otimes \cdots \otimes a_{m}\right) \otimes\left(y_{1} \otimes \cdots \otimes y_{m}\right)\right) \\
& =\widehat{\theta}_{\sigma^{-1} \vec{n}}\left(\left(a_{\sigma(1)} \otimes \cdots \otimes a_{\sigma(m)}\right) \otimes\left(y_{\sigma(1)} \otimes \cdots \otimes y_{\sigma(m)}\right)\right) \\
& = \pm \theta\left(a_{\sigma(1)} \otimes y_{\sigma(1)}\right) \otimes \cdots \otimes \theta\left(a_{\sigma(m)} \otimes y_{\sigma(m)}\right) \\
& = \pm \sigma\left(n_{\sigma(1)}, \ldots, n_{\sigma(m)}\right)\left(\theta\left(a_{1} \otimes y_{1}\right) \otimes \cdots \otimes \theta\left(a_{m} \otimes y_{m}\right)\right) \\
& =\sigma\left(n_{\sigma(1)}, \ldots, n_{\sigma(m)}\right) \widehat{\theta}_{\vec{n}}\left(\left(a_{1} \otimes \cdots \otimes a_{m}\right) \otimes\left(y_{1} \otimes \cdots \otimes y_{m}\right)\right) .
\end{aligned}
$$

This completes the proof. 
Definition 2.4 Let $H$ be a $\mathcal{P}$-Hopf algebra in $\operatorname{grMod}_{R}$ or $\mathbf{C h}_{R}$. A free algebraic $\mathcal{P}$-Hopf extension of $H$ by a generator $v$ consists of a morphism of $\mathcal{P}$-Hopf algebras $j: H \rightarrow H^{\prime}$ such that the underlying morphism of graded algebras is the inclusion map $H \hookrightarrow H \coprod T v$, where $H \coprod T v$ is the coproduct in the category of graded algebras of $H$ and of the free algebra on $v$.

We first explain in what sense free algebraic $\mathcal{P}$-Hopf extensions truly are free, in the nondifferential setting.

Proposition 2.5 Let $\mathbf{C}=\operatorname{grMod}_{R}$. Let $\left(\mathcal{Q}, \psi_{\mathcal{Q}}, \varepsilon_{\mathcal{Q}}, \Delta_{\mathcal{Q}}, e_{\mathcal{Q}}\right)$ be a level-comonoidal $\mathcal{P}$-co-ring (cf, Definition 2.1) that is free as a $\mathcal{P}$-bimodule, generated by $\mathcal{X}$. Let $H \amalg T v$ be a free algebraic $\mathcal{P}-H o p f$ extension of a $\mathcal{P}-H o p f$ algebra $H$, and let $H^{\prime}$ be another $\mathcal{P}-$ Hopf algebra.

For all $\theta \in(\mathcal{P}, \mathcal{Q})-\operatorname{PsHopf}\left(H, H^{\prime}\right)$ and $\lambda \in \operatorname{Mod}_{R}^{\Sigma}\left(\mathcal{L}(R \cdot v) \diamond \mathcal{X}, \mathcal{T}\left(H^{\prime}\right)\right)$, there is a unique morphism

$$
\widehat{(\theta+\lambda)} \in(\mathcal{P}, \mathcal{Q})-\operatorname{PsHopf}\left(H \coprod T v, H^{\prime}\right)
$$

extending $\theta$ and $\lambda$.

The proof of this proposition, which is somewhat technical, is in the appendix.

Corollary 2.6 Let $\left(\mathcal{Q}, \psi_{\mathcal{Q}}, \varepsilon_{\mathcal{Q}}, \Delta_{\mathcal{Q}}, e_{\mathcal{Q}}\right)$ be as in the statement of Proposition 2.5. Let $H$ and $H^{\prime}$ be $\mathcal{P}-$ Hopf algebras. If the underlying algebra of $H$ is free on a free graded $R$-module $V$ that is bounded below, then for all $\lambda \in \operatorname{grMod}_{R}^{\Sigma}\left(\mathcal{L}(V) \diamond \mathcal{X}, \mathcal{T}\left(H^{\prime}\right)\right)$, there is a unique morphism

$$
\widehat{\lambda} \in(\mathcal{P}, \mathcal{Q})-\operatorname{PsHopf}\left(H, H^{\prime}\right)
$$

extending $\lambda$.

More informally, we can say that if $\mathcal{P}$ is free as a bimodule, then a pseudo- $\mathcal{P}-$ Hopf algebra map with domain free as an algebra is specified by its values on generators of $\mathcal{P}$ and of the domain.

Proof The proof proceeds by induction on degree of elements in a basis of $V$, starting in the lowest degree $k$ for which $V_{k} \neq 0$, applying Proposition 2.5 at each step. Here, $\theta$ is taken to be the unique morphism with domain 0 . 


\subsection{Application to the Alexander-Whitney co-ring}

Specializing to the case where $\mathcal{P}=\mathcal{A}$, the associative operad, and $\mathcal{Q}=\mathcal{F}$, the Alexander-Whitney co-ring (cf, Section 1.3), we explain how to verify that a pseudo$\mathcal{A}$-Hopf morphism with free domain respects differential structure. The proof of Theorem 1.14 relies implicitly on the following proposition, which comes in handy later in this article as well.

Recall the notion of a family $\mathfrak{F}(\theta)$ induced by $\theta \in(\mathcal{A}, \mathcal{F})-\operatorname{Coalg}\left(C, C^{\prime}\right)$ from $(1-5)$ and of a derivation $\mathcal{D}(t): \mathcal{T}(A) \rightarrow \mathcal{T}(B)$ induced by a morphism $t: A \rightarrow B$ of graded $R$-modules (Definition 1.4).

Proposition 2.7 Let $\mathbf{C}=\mathbf{C h}_{R}$. Let $H \bigsqcup T v$ be a free algebraic $\mathcal{A}-$ Hopf extension of an $\mathcal{A}$-Hopf algebra $H$, and let $H^{\prime}$ be another $\mathcal{A}-$ Hopf algebra. Let $\Delta$ and $\Delta^{\prime}$ denote the comultiplications and $d$ and $d^{\prime}$ the differentials on $H \amalg T v$ and on $H^{\prime}$. Let

$$
\theta \in(\mathcal{A}, \mathcal{F})-\operatorname{PsHopf}\left(H, H^{\prime}\right)
$$

with induced family $\mathfrak{F}(\theta)=\left\{\theta_{k} \mid k \geq 1\right\}$.

For any set $\left\{\lambda_{k} \in \mathcal{T}\left(H^{\prime}\right)(k) \mid k \geq 1\right\}$ such that for all $k$,

$$
\mathcal{D}\left(d^{\prime}\right)_{k} \lambda_{k}-\mathcal{D}\left(\bar{\Delta}^{\prime}\right)_{k-1} \lambda_{k-1}=\theta_{k}(d v)-\sum_{i+j=k}\left(\theta_{i} \otimes \theta_{j}\right) \bar{\Delta}(v),
$$

$\theta$ can be extended to

$$
\widehat{\theta} \in(\mathcal{A}, \mathcal{F})-\operatorname{PsHopf}\left(H \coprod T v, H^{\prime}\right)
$$

such that $\widehat{\theta}\left(v \otimes z_{k-1}\right)=\lambda_{k}$ for all $k$.

Thanks to this proposition, if $T V$ is a chain Hopf algebra with free underlying algebra and $H^{\prime}$ is any chain Hopf algebra, it is possible to construct monoidal morphisms in $(\mathcal{A}, \mathcal{F})-$ Coalg from $T V$ to $H^{\prime}$ by induction on the generators $V$.

Proof The family $\left\{\lambda_{k} \mid k \geq 1\right\}$ is equivalent to a morphism of symmetric sequences of graded $R$-modules $\lambda: \mathcal{L}(R \cdot v) \diamond \mathcal{S} \rightarrow \mathcal{T}\left(H^{\prime}\right)$. We can therefore apply Proposition 2.5 to obtain $\widehat{\theta}$ as a morphism of nondifferential objects. On the other hand, as we can see from the definition of $\partial_{\mathcal{F}}$, the hypothesis on the family $\left\{\lambda_{k}\right\}$ is exactly the condition that must be satisfied for $\widehat{\theta}$ to be a differential map.

We recall now the relationship between the functor Ind (Theorem 1.14) and the monoidal structures on the source and target categories, as developed in [6] and [8]. 
Lemma 2.8 (Hess, Parent, Scott and Tonks [8]) The induction functor

$$
\text { Ind: }(\mathcal{A}, \mathcal{F})-\text { Coalg } \rightarrow \mathcal{A}-\mathbf{A l g}
$$

is comonoidal, ie, there is a natural transformation of functors into associative chain algebras

$$
q: \operatorname{Ind}(-\curlywedge-) \rightarrow \operatorname{Ind}(-) \otimes \operatorname{Ind}(-)
$$

which is given by the Milgram equivalence (0-4) on objects.

Throughout the remainder of this article, we consider objects in the following category derived from $(\mathcal{A}, \mathcal{F})$-Coalg. Recall $z_{k}$ is the generator of $\mathcal{F}$ in level $k+1$, which is of degree $k$.

Definition 2.9 The objects of the weak Alexander-Whitney category $\mathbf{w F}$ are pairs $(C, \Psi)$, where $C$ is a object in $\mathcal{A}$-Coalg and $\Psi \in(\mathcal{A}, \mathcal{F})-\operatorname{Coalg}(C, C \otimes C)$ such that

$$
\Psi\left(-\otimes z_{0}\right): C \rightarrow C \otimes C
$$

is exactly the comultiplication on $C$, while

$$
\mathbf{w F}\left((C, \Psi),\left(C^{\prime}, \Psi^{\prime}\right)\right)=\left\{\theta \in(\mathcal{A}, \mathcal{F})-\operatorname{Coalg}\left(C, C^{\prime}\right) \mid \Psi^{\prime} \theta=(\theta \curlywedge \theta) \Psi\right\} .
$$

The objects of $\mathbf{w F}$ are called weak Alexander-Whitney coalgebras.

As noted in the next lemma, the cobar construction provides an important link between the weak Alexander-Whitney category and the following category of algebras endowed with comultiplications, which are not necessarily coassociative.

Definition 2.10 The objects of the weak Hopf algebra category wH are pairs $(A, \psi)$, where $A$ is a chain algebra over $R$ and $\psi: A \rightarrow A \otimes A$ is a map of chain algebras, while

$$
\mathbf{w H}\left((A, \psi),\left(A^{\prime}, \psi^{\prime}\right)\right)=\left\{f \in \mathcal{A}-\mathbf{A l g}\left(A, A^{\prime}\right) \mid \psi^{\prime} f=(f \otimes f) \psi\right\} .
$$

Lemma 2.11 (Hess, Parent, Scott and Tonks [8]) The cobar construction extends to a functor

$$
\widetilde{\Omega}: \mathbf{w F} \rightarrow \mathbf{w H},
$$

given by $\widetilde{\Omega}(C, \Psi)=(\Omega C, q \operatorname{Ind}(\Psi))$, where $\operatorname{Ind}(\Psi): \Omega C \rightarrow \Omega(C \otimes C)$, as in Theorem 1.14, $q: \Omega(C \otimes C) \rightarrow \Omega C \otimes \Omega C$ is Milgram's equivalence (0-4) and $\widetilde{\Omega} \theta=\operatorname{Ind}(\theta): \Omega C \rightarrow \Omega C^{\prime}$ for all $\theta \in \mathbf{w F}\left((C, \Psi),\left(C^{\prime}, \Psi^{\prime}\right)\right)$.

Motivated by topology, we are particularly interested in those objects $(C, \Psi)$ of $\mathbf{w F}$ for which $\widetilde{\Omega}(C, \Psi)$ is actually a strict Hopf algebra, ie, such that $q \operatorname{Ind}(\Psi)$ is coassociative. 
Definition 2.12 The Alexander-Whitney category $\mathbf{F}$ is the full subcategory of $\mathbf{w F}$ such that $(C, \Psi)$ is an object of $\mathbf{F}$ if and only if $q \operatorname{Ind}(\Psi)$ is coassociative. We call the objects of $\mathbf{F}$ Alexander-Whitney coalgebras.

From Lemma 2.11 , it is clear that $\widetilde{\Omega}$ restricts to a functor

$$
\widetilde{\Omega}: \mathbf{F} \rightarrow \mathbf{H}
$$

where $\mathbf{H}=\mathcal{A}-\mathbf{H o p f}$ is the usual category of chain Hopf algebras.

We can now explain the topological importance of the category $\mathbf{F}$.

Theorem 2.13 (Hess, Parent, Scott and Tonks [8]) There is a functor $\widetilde{C}: \mathbf{s S e t}_{1} \rightarrow \mathbf{F}$ from the category of 1-reduced simplicial sets to the Alexander-Whitney category such that the coassociative chain coalgebra underlying $\widetilde{C}(K)$ is $C(K)$, the normalized chains on $K$. Furthermore, there is a natural quasi-isomorphism of chain algebras

$$
\widetilde{\Omega} \widetilde{C}(K) \stackrel{\simeq}{\rightarrow} C(G K)
$$

that is also itself a DCSH map.

\section{Extended multiplicative naturality of Cotor}

Let $C$ be a chain coalgebra with comultiplication $\Delta$. If $(M, v)$ and $\left(M^{\prime}, v^{\prime}\right)$ are right and left $C$-comodules, respectively, then their cotensor product over $C$ is

$$
M \square_{C} M^{\prime}=\operatorname{ker}\left(M \otimes M^{\prime} \stackrel{\nu \otimes 1-1 \otimes v^{\prime}}{\longrightarrow} M \otimes C \otimes M^{\prime}\right) .
$$

In particular, if we endow the ground ring $R$ with its trivial left $C$-comodule structure, then

$$
M \square_{C} R \cong\{x \in M \mid v(x)=x \otimes 1\},
$$

so that $M \square_{C} R$ can be seen as a graded submodule of $M$, which we can think of as the "cofixed points" of the coaction $v$.

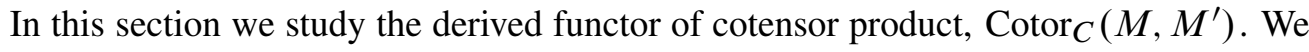
begin by recalling the formula for Cotor in terms of one-sided cobar constructions, from which it is immediately clear that Cotor is natural in all three variables, with respect to morphisms of comodules over a fixed coalgebra and with respect to morphisms of coalgebras. In [5], Gugenheim and Munkholm proved an "extended naturality" result for Cotor, ie, that Cotor is actually functorial with respect to a much larger class of morphisms. In Section 3.2 we reformulate Gugenheim and Munkholm's result in operadic language. 
We show in Section 3.1 that if $H$ is a chain Hopf algebra and $B$ is a (left) $H$-comodule algebra, then $\operatorname{Cotor}_{H}(R, B)$ admits a graded multiplicative structure, which is natural in both variables, with respect to morphisms of comodule algebras over fixed Hopf algebras and with respect to morphisms of Hopf algebras. We then prove in Section 3.2 that there is a larger class of morphisms, the class of comodule-algebra maps up to strong homotopy (CASH maps), with respect to which the multiplicative structure of $\operatorname{Cotor}_{H}(R, B)$ is natural, ie, we establish "extended multiplicative naturality" of Cotor, which plays an important role in Section 5 and Section 6.

\subsection{Cotor: definition and naturality}

The derived functor of cotensor product, Cotor, can be calculated in terms of the following complex.

Definition 3.1 Let $C$ be a simply-connected chain coalgebra, and let $M$ be a right $C$-comodule. The one-sided cobar construction $M \otimes_{t_{\Omega}} \Omega C$ is the chain complex with underlying graded $R$-module $M \otimes T s^{-1} C_{+}$and with differential $D_{\Omega}$ given by

$$
D_{\Omega}(x \otimes w)=d x \otimes w+(-1)^{x} x \otimes d_{\Omega} w+(-1)^{x_{i}} x_{i} \otimes\left(s^{-1} c^{i} \cdot w\right),
$$

where $x \in M, w \in \Omega C, d$ is the differential on $M, d_{\Omega}$ is the cobar construction differential (cf Notation and Conventions), $v(x)=x_{i} \otimes c^{i}$ and $s^{-1} 1:=0$.

There is an analogous definition of $\Omega C \otimes_{t_{\Omega}} N$ for any left $C$-comodule $N$.

Remark 3.2 If $M=C$ or $N=C$, we obtain the usual acyclic cobar constructions:

$$
C \otimes_{t_{\Omega}} \Omega C \quad \text { and } \quad \Omega C \otimes_{t_{\Omega}} C .
$$

Remark 3.3 The formula in the definition above makes it clear that there are functors

$$
-\otimes \Omega C: \operatorname{Comod}_{C} \rightarrow \mathbf{C h}_{R}
$$

and

$$
\Omega C \otimes-:{ }_{C} \operatorname{Comod} \rightarrow \mathrm{Ch}_{R} .
$$

One-sided cobar constructions can be applied to calculations of Cotor, the derived functor of the cotensor product. Let $C$ be a connected coalgebra, and let $M$ and $N$ be right and left comodules over $C$, respectively. Then, as shown in eg [3],

$$
\operatorname{Cotor}^{C}(M, N)=\mathrm{H}_{*}\left(\left(M \otimes_{t_{\Omega}} \Omega C\right) \otimes_{\Omega C}\left(\Omega C \otimes_{t_{\Omega}} N\right)\right) .
$$

It follows from the previous remark that Cotor is a bifunctor

$$
\text { Cotor: } \operatorname{Comod}_{C} \times{ }_{C} \operatorname{Comod} \rightarrow \operatorname{grMod}_{R} .
$$


We think of this as the linear naturality of Cotor.

Remark 3.4 We can also use the cobar construction to define the homotopy fiber of a morphism of coaugmented chain coalgebras $f: C^{\prime} \rightarrow C$. Consider the projection $\pi: C \otimes_{t_{\Omega}} \Omega C \rightarrow C$, which is a surjective morphism of chain complexes with contractible source, and therefore an acceptable candidate for a fibrant replacement of the coaugmentation $\eta: R \rightarrow C$. Consequently, we can define the homotopy fiber of $f$ to be the pullback of

$$
C \otimes_{t_{\Omega}} \Omega C \stackrel{\pi}{\rightarrow} C \stackrel{f}{\leftarrow} C^{\prime},
$$

ie $C^{\prime} \otimes_{t_{\Omega}} \Omega C$. The homology of the homotopy fiber of $f$ is thus exactly $\operatorname{Cotor}^{C}\left(C^{\prime}, R\right)$.

Let $H$ be a chain Hopf algebra. Recall that a chain algebra $B$ that is also an $H-$ comodule is an $H$-comodule algebra if the $H$-coaction map is a morphism of chain algebras. In [12], Miller proved the existence of a natural chain algebra structure on the one-sided cobar construction $\Omega H \otimes_{t_{\Omega}} B$, for any commutative Hopf algebra $H$ and any left $H$-comodule algebra $B$. Here we dualize [4, Theorem 4.1], obtaining a generalization of Miller's result to any chain Hopf algebra $H$. As a consequence, $\operatorname{Cotor}^{H}(R, B)$ admits a natural multiplicative structure for any Hopf algebra $H$ and any $H$-comodule algebra $B$.

We begin by considering a special case: the acyclic cobar construction. Though it would be possible to prove the next proposition and its corollaries by appealing to [4, Theorem 4.1] and then dualizing, we prefer to give a direct, constructive proof, since the explicit formulas we provide are much simpler than those in the dual case and prove quite useful.

Proposition 3.5 If $H$ is any chain Hopf algebra, then the free left $\Omega H$-module structure on $\Omega H \otimes_{t_{\Omega}} H$ can be extended to a chain algebra structure such that

$(1 \otimes c)\left(s^{-1} a \otimes 1\right)=(-1)^{(a+1) c} s^{-1} a \otimes c+(-1)^{c} s^{-1}(c \cdot a) \otimes 1+(-1)^{c+a c^{i}} s^{-1}\left(c_{i} \cdot a\right) \otimes c^{i}$

for all $a, c \in H$, where $\bar{\Delta}(c)=c_{i} \otimes c^{i}$ and

$$
(1 \otimes c)(1 \otimes e)=1 \otimes c \cdot e
$$

for all $c, e \in H$.

Proof Given the multiplication as partially defined in the statement of the proposition, we extend it to all of $\Omega H \otimes_{t_{\Omega}} H$ by associativity, which is possible since $\Omega H$ is free as an algebra on $s^{-1} H_{+}$. Hence all that we must do is verify that $D_{\Omega}$ is a derivation 
with respect to this product. We do the second case and leave the first, the proof of which is quite similar, to the reader.

If image of $c$ and $e$ under the reduced comultiplication are $c_{i} \otimes c^{i}$ and $e_{j} \otimes e^{j}$, respectively, then the image of $c \cdot e$ under the unreduced comultiplication is

$$
\begin{aligned}
(c \cdot e)_{k} \otimes(c \cdot e)^{k}= & c \cdot e \otimes 1+1 \otimes c \cdot e \\
& +c \otimes e+(-1)^{e c} e \otimes c \\
& +c_{i} \otimes c^{i} \cdot e+(-1)^{e c_{i}} c_{i} \cdot e \otimes c^{i} \\
& +c \cdot e_{j} \otimes e^{j}+(-1)^{e_{j} c} e_{j} \otimes c \cdot e^{j} \\
& +(-1)^{e_{j} c^{i}} c_{i} \cdot e_{j} \otimes c^{i} \cdot e^{j}
\end{aligned}
$$

Consequently,

$$
\begin{aligned}
D_{\Omega}(1 \otimes c \cdot e)= & 1 \otimes d(c \cdot e)-s^{-1}(c \cdot e) \otimes 1 \\
& -s^{-1} c \otimes e-(-1)^{e c} s^{-1} e \otimes c \\
& -s^{-1} c_{i} \otimes c^{i} \cdot e-(-1)^{e c_{i}} s^{-1}\left(c_{i} \cdot e\right) \otimes c^{i} \\
& -s^{-1}\left(c \cdot e_{j}\right) \otimes e^{j}-(-1)^{e_{j} c} s^{-1} e_{j} \otimes c \cdot e^{j} \\
& -(-1)^{e_{j} c^{i}} s^{-1}\left(c_{i} \cdot e_{j}\right) \otimes c^{i} \cdot e^{j} .
\end{aligned}
$$

On the other hand

$$
D_{\Omega}(1 \otimes c) \cdot(1 \otimes e)=1 \otimes d c \cdot e-s^{-1} c \otimes e-s^{-1} c_{i} \otimes c^{i} \cdot e,
$$

while

$(-1)^{c}(1 \otimes c) \cdot D_{\Omega}(1 \otimes e)$

$$
\begin{aligned}
= & (-1)^{c} 1 \otimes c \cdot d e+(-1)^{c}(1 \otimes c) \cdot\left(-s^{-1} e \otimes 1-s^{-1} e_{j} \otimes e^{j}\right) \\
= & -(-1)^{c e} s^{-1} e \otimes c-s^{-1}(c \cdot e) \otimes 1 \\
& -(-1)^{e c^{i}} s^{-1}\left(c_{i} \cdot e\right) \otimes c^{i}-(-1)^{e_{j} c} s^{-1} e_{j} \otimes c \cdot e^{j} \\
& -s^{-1}\left(c \cdot e_{j}\right) \otimes e^{j}-(-1)^{e_{j} c^{i}} s^{-1}\left(c_{i} \cdot e_{j}\right) \otimes c^{i} \otimes e^{j} .
\end{aligned}
$$

It is now obvious that

$$
D_{\Omega}(1 \otimes c \cdot e)=D_{\Omega}(1 \otimes c) \cdot(1 \otimes e)+(-1)^{c}(1 \otimes c) \cdot D_{\Omega}(1 \otimes e) .
$$

This proposition admits the following generalization, the proof of which is essentially identical to that of the proposition.

Algebraic 83 Geometric Topology, Volume 7 (2007) 
Corollary 3.6 If $H$ is any chain Hopf algebra and $B$ is a left $H$-comodule algebra, with coaction map $v$, then the free left $\Omega H$-module structure on $\Omega H \otimes_{t_{\Omega}} B$ can be extended to a chain algebra structure such that

$(1 \otimes b)\left(s^{-1} a \otimes 1\right)=(-1)^{(a+1) b} s^{-1} a \otimes b+(-1)^{c} s^{-1}(c \cdot a) \otimes 1+(-1)^{b+a b^{i}} s^{-1}\left(c_{i} \cdot a\right) \otimes b^{i}$

for all $a \in H, b \in B$, where $v(b)=c \otimes 1+1 \otimes b+c_{i} \otimes b^{i}$ and

$$
(1 \otimes b)\left(1 \otimes b^{\prime}\right)=1 \otimes b \cdot b^{\prime}
$$

for all $b, b^{\prime} \in B$.

An analogous results clearly holds for right comodule algebras as well.

The multiplicative structure defined above is easily seen to be natural, in the following sense. Let $f: H \rightarrow H^{\prime}$ be a morphism of chain Hopf algebras. Let $(B, v)$ be a left $H$-comodule algebra, and let $\left(B^{\prime}, v^{\prime}\right)$ be a left $H^{\prime}$-comodule algebra. Let $g: B \rightarrow B^{\prime}$ be morphism of chain algebras such that $(f \otimes g) v=v^{\prime} g$. It is easy to check that the chain map

$$
\Omega f \otimes g: \Omega H \otimes_{t_{\Omega}} B \rightarrow \Omega H^{\prime} \otimes_{t_{\Omega}} B^{\prime}
$$

respects the multiplicative structure defined in Corollary 3.6.

Let $\mathbf{C A}^{\ell}$ denote the following category. Objects are pairs $(H ; B)$, where $H$ is a chain Hopf algebra and $B$ is a left $H$-comodule algebra, both over the fixed PID $R$. A morphism from $(H ; B)$ to $\left(H^{\prime} ; B^{\prime}\right)$ is a pair $(f ; g)$, where $f: H \rightarrow H^{\prime}$ is a morphism of chain Hopf algebras and $g: B \rightarrow B^{\prime}$ is a chain algebra map such that $(f \otimes g) v=v^{\prime} g$, where $v$ and $v^{\prime}$ are the coactions on $B$ and $B^{\prime}$, respectively. The analogous category for right comodule algebras is denoted $\mathbf{C A}^{r}$.

Corollary 3.7 Let $\mathbf{c h} \mathbf{A}$ denote the category of chain algebras over $R$. There are functors

$$
\Omega^{\ell}(-;-): \mathbf{C A}^{\ell} \rightarrow \mathbf{c h} \mathbf{A}
$$

defined by $\Omega(H ; B)=\Omega H \otimes_{t_{\Omega}} B$ (with the multiplicative structure of Corollary 3.6) and $\Omega(f ; g)=\Omega f \otimes g$ and

$$
\Omega^{r}(-;-): \mathbf{C A}^{r} \rightarrow \mathbf{c h} \mathbf{A}
$$

defined by $\Omega(H ; B)=B \otimes_{t_{\Omega}} \Omega H$ (with the multiplicative structure analogous to that of Corollary 3.6) and $\Omega(f ; g)=g \otimes \Omega f$.

We show in Section 3.2 that the algebra structure on $\Omega H \otimes_{t_{\Omega}} B$ is actually natural with respect to a bigger class of morphisms than those of the category $\mathbf{C A}^{\ell}$. This extended naturality of the algebra structure of $\Omega H \otimes_{t_{\Omega}} B$ plays a crucial role in Section 6. 
Corollary 3.8 Let grA denote the category of graded algebras over $R$. The functor Cotor restricts and corestricts to functors

$$
\operatorname{Cotor}^{(-)}(R ;-): \mathbf{C A}^{\ell} \rightarrow \mathbf{g r A}
$$

and

$$
\operatorname{Cotor}^{(-)}(-; R): \mathbf{C A}^{r} \rightarrow \mathbf{g r A}
$$

This is the multiplicative naturality of Cotor.

Proof From the definition of Cotor (3-1), we see that if $B$ is a left $H$-comodule algebra, then

$$
\operatorname{Cotor}^{H}(R, B)=\mathrm{H}_{*}\left(\Omega H \otimes_{t_{\Omega}} B\right) .
$$

The previous corollary implies that there is a natural graded algebra structure on $\mathrm{H}_{*}\left(\Omega H \otimes_{t_{\Omega}} B\right)$. The right-comodule case works similarly.

Remark 3.9 As a consequence of Proposition 3.5, we obtain that $\Omega H \otimes_{t_{\Omega}} H$ is itself an $H$-comodule algebra. To establish this fact, we must show that the following diagram commutes.

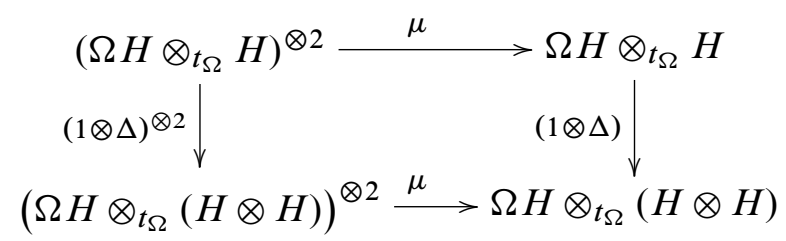

Here, $\Delta$ is the comultiplication on $H$ and $\mu$ denotes the multiplication defined in the statements of Proposition 3.5 and Corollary 3.6. The left $H$-comodule structure on $H \otimes H$ is given by $\Delta \otimes 1$. It suffices to check that this diagram commutes on elements of the form $1 \otimes c \otimes s^{-1} a \otimes 1$, which is not a difficult computation. The coassociativity of $\Delta$ plays a crucial role in this verification.

Analagously, $H \otimes_{t_{\Omega}} \Omega H$ is also an $H$-comodule algebra.

\subsection{Maps of comodules up to strong homotopy}

In this article we need relative versions of the results from [8] cited in Section 1.3, to establish conditions under which there is a multiplicative map between one-sided cobar constructions of the sort considered in Corollary 3.6. As a consequence, we obtain Proposition 3.19, which is both a multiplicative generalization of the extended naturality of Cotor, due to Gugenheim and Munkholm [5] (Theorem 3.13 below), and an extended version of the multiplicative naturality of Cotor (Corollary 3.8 above). 
Let $C$ and $C^{\prime}$ be connected chain coalgebras. Recall from Section 1.3 that though every chain coalgebra map $f: C \rightarrow C^{\prime}$ induces a chain algebra map $\Omega f: \Omega C \rightarrow \Omega C^{\prime}$, not all chain algebra maps $\varphi: \Omega C \rightarrow \Omega C^{\prime}$ are so induced. In particular, the category of chain coalgebras can be seen as a wide, but not full, subcategory of the category DCSH, so that the morphisms in DCSH can be considered as "weak" chain coalgebra morphisms.

In this section we consider an analogous weakening of the morphisms in the category $\mathbf{C A}^{r}$ defined in Section 3.1, for which we provide equivalent chain-level and operadic definitions, both of which are quite useful. The operadic definition serves to facilitate the proofs of the existence results (Proposition 3.16 and Proposition 3.18) that play a key role in Section 5.

The reader who is not interested in the fine details of our constructions and existence results can safely limit his perusal of this section only to the definition of the category CASH (Definition 3.14) and to Proposition 3.18.

Let $\mathbf{C}$ denote either the category of graded $R$-modules or the category of chain complexes over $R$. Let $\mathbf{C}^{\Sigma_{+}}$denote the category of shifted symmetric sequences in C. An object $\mathcal{X}$ of $\mathbf{C}^{\Sigma_{+}}$is a family $\{\mathcal{X}(n) \in \mathbf{C} \mid n \geq 0\}$ of objects in $\mathbf{C}$ such that $\mathcal{X}(n)$ admits a right action of the symmetric group $\Sigma_{n-1}$, for all $n>0$ and such that $\mathcal{X}(0)=0$. A morphism in $\mathbf{C}^{\Sigma_{+}}$from $\mathcal{X}$ to $\mathcal{Y}$ consists of a family

$$
\left\{\varphi_{n} \in \mathbf{C}(\mathcal{X}(n), \mathcal{Y}(n)) \mid \varphi_{n} \quad \text { is } \Sigma_{n-1} \text {-equivariant, } n \geq 1\right\} .
$$

There is a faithful functor

$$
\mathcal{T}_{r}: \mathbf{C} \times \mathbf{C} \rightarrow \mathbf{C}^{\Sigma_{+}}
$$

where, for all $n, \mathcal{T}_{r}(A, B)(n)=A \otimes B^{\otimes n-1}$, where $\Sigma_{n-1}$ acts by permuting the factors of $B$.

The following useful operation on symmetric sequences in the image of $\mathcal{T}_{r}$ is a shifted version of the notion of derivation of symmetric sequences in the image of $\mathcal{T}$ (Definition $1.4)$.

Definition 3.10 Let $f, s: A \rightarrow B$ and $g, h, t: C \rightarrow D$ be homogenous linear maps of graded $R$-modules, such that $f, g$ and $h$ are homogeneous of degree 0 , and $s$ and $t$ are homogeneous of degree $m$. The $(f, g, h)$-derivation of shifted symmetric sequences induced by $s$ and $t$ is the morphism of symmetric sequences

$$
\mathcal{D}_{(f, g, h)}(s, t): \mathcal{T}_{r}(A, C) \rightarrow \mathcal{T}_{r}(B, D)
$$


that is of degree $m$ in each level and that is defined as follows in level $n$.

$$
\mathcal{D}_{(f, g, h)}(s, t)_{n}=s \otimes h^{\otimes n-1}+\sum_{j=0}^{n-2} f \otimes g^{\otimes j} \otimes t \otimes h^{\otimes n-j-2} .
$$

When $A=B, C=D$ and $f=\operatorname{Id}_{A}$ and $g=\operatorname{Id}_{C}=h$, we simplify notation and write $\mathcal{D}(s, t)$ for the $\left(\operatorname{Id}_{A}, \operatorname{Id}_{C}, \operatorname{Id}_{C}\right)$-derivation induced by $s$ and $t$.

It is obvious that there is again a level monoidal structure $\left(\mathbf{C}^{\Sigma_{+}}, \otimes, \mathcal{C}\right)$, where $(\mathcal{X} \otimes$ $\mathcal{Y})(n)=\mathcal{X}(n) \otimes \mathcal{Y}(n)$, endowed with the diagonal action of $\Sigma_{n-1}$, and $\mathcal{C}(n)=R$, endowed with the trivial $\Sigma_{n-1}$-action. By proofs analogous to those in [10, section II.1.8], we can show that the category $\mathbf{C}^{\boldsymbol{\Sigma}_{+}}$also admits a right action by the monoidal category $\left(\mathbf{C}^{\Sigma}, \diamond, \mathcal{J}\right)$, ie there is a bifunctor

$$
\mathbf{C}^{\Sigma_{+}} \times \mathbf{C}^{\Sigma} \rightarrow \mathbf{C}^{\Sigma_{+}}:(\mathcal{X}, \mathcal{Y}) \mapsto \mathcal{X} \triangleleft \mathcal{Y}
$$

defined by

$$
(\mathcal{X} \triangleleft \mathcal{Y})(n)=\coprod_{\substack{k \geq 1 \\ \vec{\imath} \in I_{k, n}}} \mathcal{X}(k) \underset{\Sigma_{k-1}}{\otimes}\left(Y\left(i_{1}\right) \otimes \cdots \otimes Y\left(i_{k}\right)\right) \underset{\Sigma_{\vec{\imath}-\vec{e}_{1}}}{\otimes} R\left[\Sigma_{n-1}\right] .
$$

Here, $\Sigma_{k-1}$ acts on $Y\left(i_{1}\right) \otimes \cdots \otimes Y\left(i_{k}\right)$ by permuting $Y\left(i_{2}\right) \otimes \cdots \otimes Y\left(i_{k}\right)$, while $\vec{\imath}-\vec{e}_{1}=\left(i_{1}-1, i_{2}, \ldots, i_{k}\right)$. Furthermore, there is a natural isomorphism $\mathcal{X} \triangleleft(\mathcal{Y} \diamond \mathcal{Z}) \cong$ $(\mathcal{X} \triangleleft \mathcal{Y}) \triangleleft \mathcal{Z}$ for all shifted symmetric sequences $\mathcal{X}$ and all symmetric sequences $\mathcal{Y}$ and $\mathcal{Z}$.

Let $\mathcal{P}$ be an operad, with multiplication map $\gamma: \mathcal{P} \diamond \mathcal{P} \rightarrow \mathcal{P}$, and let $\mathcal{X}$ be a shifted symmetric sequence. We say that $\mathcal{X}$ is a shifted right $\mathcal{P}$-module if there is a morphism of shifted symmetric sequences $\rho: \mathcal{X} \triangleleft \mathcal{P} \rightarrow \mathcal{X}$ such that

$$
\rho(\rho \triangleleft 1)=\rho(1 \triangleleft \gamma):(\mathcal{X} \triangleleft \mathcal{P}) \triangleleft P \cong \mathcal{X} \triangleleft(\mathcal{P} \diamond \mathcal{P}) \rightarrow \mathcal{X} .
$$

A morphism of shifted right $\mathcal{P}$-modules is a morphism of the underlying shifted symmetric sequences that commutes with the right action maps. We write $\operatorname{Mod}_{\mathcal{P}}^{+}$ for the category of shifted right $\mathcal{P}$-modules and their morphisms. Given a shifted symmetric sequence $\mathcal{X}$ that is a shifted right $\mathcal{P}$-module and a symmetric sequence $\mathcal{Y}$ that is a left $\mathcal{P}$-module (in the usual sense), we define $\mathcal{X}_{\mathcal{P}} \mathcal{Y}$ to be the obvious coequalizer.

Definition 3.11 Let $\theta \in(\mathcal{A}, \mathcal{F})-\operatorname{Coalg}\left(C, C^{\prime}\right)$, inducing $\operatorname{Ind}(\theta) \in \mathcal{A}-\operatorname{Alg}\left(\Omega C, \Omega C^{\prime}\right)$ and therefore the structure of a right $\Omega C$-module on $\Omega C^{\prime}$. Suppose that $M$ is a right 
$C$-comodule and $M^{\prime}$ is a right $C^{\prime}$-comodule. A map of right $\Omega C$-modules

$$
h: M \otimes_{t_{\Omega}} \Omega C \rightarrow M^{\prime} \otimes_{t_{\Omega}} \Omega C^{\prime},
$$

is a comodule map up to strong homotopy with respect to $\theta$ from $M$ to $M^{\prime}$.

Abusing terminology somewhat, we say that a chain map $g: M \rightarrow M^{\prime}$ is a comodule map up to strong homotopy if there is such an $h$ satisfying

$$
h(x \otimes 1)-g(x) \otimes 1 \in M_{<\operatorname{deg} x}^{\prime} \otimes \Omega C^{\prime}
$$

for all $x \in M$.

A "module" version of Theorem 1.14 holds for right comodules. The proof proceeds by straightforward generalization of the absolute case. Before stating the theorem, we remark that if $C$ is a coassociative chain coalgebra and $M$ is a right $C$-comodule, then $\mathcal{T}_{r}(M, C)$ is naturally a shifted right $\mathcal{A}$-module. Observe that there is an isomorphism of graded $R$-modules

$$
\begin{aligned}
& \mathcal{T}_{r}(M, C)(k) \underset{\Sigma_{k-1}}{\otimes} \mathcal{A}\left(i_{1}\right) \otimes \cdots \otimes \mathcal{A}\left(i_{k}\right) \\
\cong & \left(M \otimes \mathcal{A}\left(i_{1}\right)\right) \otimes\left(\mathcal{T}(C)(k-1) \underset{\Sigma_{k-1}}{\otimes} \mathcal{A}\left(i_{2}\right) \otimes \cdots \otimes \mathcal{A}\left(i_{k}\right)\right) .
\end{aligned}
$$

To define a shifted right $\mathcal{A}$-module structure on $\mathcal{T}_{r}(M, C)$, we use the right $\mathcal{A}$-module structure on $\mathcal{T}(C)$ coming from its coalgebra structure, as well as the fact that for all $m \geq 1$, the comodule map $v: M \rightarrow M \otimes C$ induces a $\Sigma_{m-1}$-equivariant map

$$
v^{(m)}: M \otimes \mathcal{A}(m) \rightarrow M \otimes C^{\otimes m-1}:\left(x \otimes \delta^{(m)}\right) \mapsto\left(\operatorname{Id}_{M} \otimes \Delta^{(m-2)}\right) v(x) .
$$

In other words, $\mathcal{T}_{r}$ induces a functor from the category of pairs $(M, C)$, where $C$ is a coassociative coalgebra and $M$ is a right $C$-comodule, to the category of shifted right $\mathcal{A}$-modules. We can now state the "module" version of Theorem 1.14.

Proposition 3.12 Let $\theta \in(\mathcal{A}, \mathcal{F})-\operatorname{Coalg}\left(C, C^{\prime}\right)$, inducing $\operatorname{Ind}(\theta) \in \mathcal{A}-\mathbf{A} \lg \left(\Omega C, \Omega C^{\prime}\right)$. If $M$ is a right $C$-comodule and $M^{\prime}$ is a right $C^{\prime}$-comodule, then there is a natural bijection

$$
\operatorname{Ind}^{+}: \operatorname{Mod}_{\mathcal{A}}^{+}\left(\mathcal{T}_{r}(M, C) \underset{\mathcal{A}}{\triangleleft} \mathcal{F}, \mathcal{T}_{r}\left(M^{\prime}, C^{\prime}\right)\right) \rightarrow \operatorname{Mod}_{\Omega C}\left(M \otimes_{t_{\Omega}} \Omega C, M^{\prime} \otimes_{t_{\Omega}} \Omega C^{\prime}\right)
$$

specified by

$$
\operatorname{Ind}^{+}(\omega)(x)=\sum_{k \geq 1}\left(\operatorname{Id}_{M^{\prime}} \otimes\left(s^{-1}\right)^{\otimes k-1}\right) \omega\left(x \otimes z_{k-1}\right)
$$

for all $x \in M$. 
To see why the formula above makes sense, note that

$$
\omega\left(x \otimes z_{k-1}\right) \in \mathcal{T}_{r}\left(M^{\prime}, C^{\prime}\right)(k)=M^{\prime} \otimes\left(C^{\prime}\right)^{\otimes k-1} .
$$

Furthermore, since $M \otimes_{t_{\Omega}} \Omega C$ is a free right $\Omega C$-module, the specification in Proposition 3.12 suffices to imply that $\operatorname{Ind}^{+}(\omega)(x \otimes v)=\operatorname{Ind}^{+}(\omega)(x) \cdot \operatorname{Ind}(\theta)(v)$ for all $x \in M$ and $v \in \Omega C$.

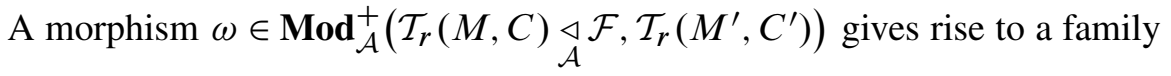

$$
\mathfrak{F}^{+}(\omega)=\left\{\omega_{k}=\omega\left(-\otimes z_{k}\right): M \rightarrow M^{\prime} \otimes\left(C^{\prime}\right)^{\otimes k} \mid \operatorname{deg} \omega_{k}=k, k \geq 0\right\} .
$$

Specifying a family $\mathfrak{F}^{+}(\omega)$ is equivalent to specifying a morphism of shifted symmetric sequences $\mathcal{L}(M) \underset{\mathcal{A}}{\triangleleft} \mathcal{F} \rightarrow \mathcal{T}_{r}\left(M^{\prime}, C^{\prime}\right)$.

Maps of left comodules up to strong homotopy are defined analogously. A version of the proposition above, expressed in terms of a functor $\mathcal{T}_{\ell}$, holds for left comodules as well.

Comodule maps up to strong homotopy are interesting because of their role in extending the linear naturality of Cotor (cf (3-1)), first established by Gugenheim and Munkholm in [5] (dual of Theorem 3.5). This extended naturality can be expressed as follows in the language we have developed above.

Theorem 3.13 (Gugenheim and Munkholm [5]) Let $\theta \in(\mathcal{A}, \mathcal{F})-\operatorname{Coalg}\left(C, C^{\prime}\right)$, where $C$ and $C^{\prime}$ are simply connected chain coalgebras. Let $g: M \rightarrow M^{\prime}$ and $h: N \rightarrow N^{\prime}$ be maps of right and left comodules, respectively, up to strong homotopy with respect to $\theta$, where $M$ and $N$ are $C$-comodules, and $M^{\prime}$ and $N^{\prime}$ are $C^{\prime}-$ comodules. Then there is a natural induced morphism of graded $R$-modules

$$
\operatorname{Cotor}^{\theta}(g, h): \operatorname{Cotor}^{C}(M, N) \rightarrow \operatorname{Cotor}^{C^{\prime}}\left(M^{\prime}, N^{\prime}\right) .
$$

Furthermore if all the underlying graded modules are $R$-flat and $\theta\left(-\otimes z_{0}\right), g$, and $h$ are all quasi-isomorphisms, then $\operatorname{Cotor}^{\theta}(g, h)$ is an isomorphism.

We sketch a proof of Theorem 3.13, based on Proposition 3.12. Let

$$
\xi: \mathcal{T}_{r}(M, C) \underset{\mathcal{A}}{\triangleleft} \mathcal{F} \rightarrow \mathcal{T}\left(M^{\prime}, C^{\prime}\right) \quad \text { and } \quad \zeta: \mathcal{T}_{r}(N, C) \underset{\mathcal{A}}{\triangleleft} \mathcal{F} \rightarrow \mathcal{T}\left(N^{\prime}, C^{\prime}\right)
$$

be the morphisms of shifted $\mathcal{A}$-modules of chain complexes, corresponding to $g$ and $h$. Thus, under the hypotheses of the theorem, we can set

$$
\operatorname{Cotor}^{\theta}(g, h)=\mathrm{H}_{*}\left(\operatorname{Ind}^{+}(\xi) \otimes_{\Omega C} \operatorname{Ind}^{+}(\zeta)\right): \operatorname{Cotor}^{C}(M, N) \rightarrow \operatorname{Cotor}^{C^{\prime}}\left(M^{\prime}, N^{\prime}\right)
$$


A standard spectral sequence argument then shows that $\operatorname{Cotor}^{\theta}(g, h)$ is an isomorphism if all modules are $R$-flat and if $\mathrm{H}_{*}\left(\theta\left(-\otimes z_{0}\right)\right), \mathrm{H}_{*} g$, and $\mathrm{H}_{*} h$ are all isomorphisms. We devote the remainder of this section to establishing a framework in which to state and prove a multiplicative version of Theorem 3.13. Recall that if $H$ is a Hopf algebra, then an algebra $B$ is an $H$-comodule algebra if it is an $H$-comodule and the comodule structure maps are algebra maps. Furthermore, as seen in Corollary 3.6, a right $H$-comodule algebra $B$ naturally gives rise to a chain algebra, $B \otimes_{t_{\Omega}} \Omega H$.

We can now enlarge the category $\mathbf{C A}^{r}$ by weakening the definition of morphisms, in analogy with the passage from the category of chain coalgebras to the category DCSH.

Definition 3.14 Let CASH be the category specified as follows.

(1) Objects are pairs $(H ; B)$, where $H$ is a chain Hopf algebra and $B$ is a right $H$-comodule algebra.

(2) A morphism from an object $(H ; B)$ to an object $\left(H^{\prime} ; B^{\prime}\right)$ is a pair $(\theta ; \gamma)$, where

$$
\theta \in(\mathcal{A}, \mathcal{F})-\operatorname{PsHopf}\left(H, H^{\prime}\right)
$$

and

$$
\gamma: B \otimes_{t_{\Omega}} \Omega H \rightarrow B^{\prime} \otimes_{t_{\Omega}} \Omega H^{\prime}
$$

is a morphism of both chain algebras and $\Omega H$-modules, where the right $\Omega H$ module structure on $B^{\prime} \otimes_{t_{\Omega}} \Omega H^{\prime}$ is given by the algebra morphism

$$
\operatorname{Ind}(\theta): \Omega H \rightarrow \Omega H^{\prime} \text {. }
$$

Composition and identities are defined in the obvious manner. The morphisms in CASH are called comodule-algebra maps up to strong homotopy.

Given a morphism $(\theta ; \gamma):(H ; B) \rightarrow\left(H^{\prime} ; B^{\prime}\right)$ in CASH, let $\gamma_{0}$ denote the composite

$$
B \hookrightarrow B \otimes_{t_{\Omega}} \Omega H \stackrel{\gamma}{\rightarrow} B^{\prime} \otimes_{t_{\Omega}} \Omega H^{\prime} \stackrel{\pi}{\rightarrow} B^{\prime},
$$

where $\pi$ denotes the obvious projection. We say that a chain map $g: B \rightarrow B^{\prime}$ is a CASH map if there is a morphism $(\theta ; \gamma):(H ; B) \rightarrow\left(H^{\prime} ; B^{\prime}\right)$ in CASH such that $\gamma_{0}=g$.

Remark 3.15 Corollary 3.7 implies that the category CA embeds into CASH as a wide, but not necessarily full, subcategory.

The following relative version of Proposition 2.7 is a crucial tool for construction of CASH maps. The proof proceeds by direct, but somewhat cumbersome, generalization of Proposition 2.5, the details of which we spare the reader. We use here the shifted derivations of Definition 3.10. 
Proposition 3.16 Fix chain Hopf algebras $H$ and $H^{\prime}$ and

$$
\theta \in(\mathcal{A}, \mathcal{F})-\operatorname{PsHopf}\left(H, H^{\prime}\right)
$$

with $\mathfrak{F}(\theta)=\left\{\theta_{k} \mid k \geq 1\right\}$. Let $B$ be a right $H$-comodule algebra and $B^{\prime}$ a right $H^{\prime}$-comodule algebra such that $B$ is free as an algebra on an $R$-free graded module $V$. Then any family of morphisms of graded $R$-modules

$$
\Xi=\left\{\xi_{k}: V \rightarrow B^{\prime} \otimes\left(H^{\prime}\right)^{\otimes k} \mid \operatorname{deg} \xi_{k}=k, k \geq 0\right\}
$$

naturally induces a unique morphism of shifted right $\mathcal{A}$-modules of graded $R$-modules

$$
\widehat{\xi}: \mathcal{T}_{r}(B, H) \underset{\mathcal{A}}{\triangleleft \mathcal{F}} \rightarrow \mathcal{T}_{r}\left(B^{\prime}, H^{\prime}\right)
$$

such that $\widehat{\xi}\left(v \otimes z_{k}\right)=\xi_{k}(v)$ for all $v \in V$ and such that

$$
\operatorname{Ind}^{+}(\widehat{\xi}): B \otimes_{t_{\Omega}} \Omega H \rightarrow B^{\prime} \otimes_{t_{\Omega}} \Omega H^{\prime}
$$

is a map of graded algebras and of $\Omega H$-modules.

If, furthermore, for all $k \geq 0$ and for all $v \in V$,

(3-3) $\mathcal{D}\left(d_{B^{\prime}}, d_{H^{\prime}}\right)_{k+1} \xi_{k}(v)-\mathcal{D}\left(\bar{v}^{\prime}, \bar{\Delta}^{\prime}\right)_{k} \xi_{k-1}(v)=\widehat{\xi}_{k}(d v)-\sum_{i+j=k}\left(\widehat{\xi}_{i} \otimes \theta_{j}\right) \bar{v}(v)$,

where $\mathfrak{F}^{+}(\widehat{\xi})=\left\{\widehat{\xi}_{k} \mid k \geq 0\right\}$, then $\widehat{\xi}$ is a differential map.

The next proposition, which explains how to construct a CASH map as a sort of coproduct of CASH maps when the underlying algebras of the sources are free, is essential to the proof in Section 5 that our algebraic "homotopy fiber" has the right homology. Before stating the proposition, we need one observation about coproducts and tensor products of comodule algebras.

Remark 3.17 Suppose that $A$ and $A^{\prime}$ are right $H$-comodule algebras with coaction maps $v: A \rightarrow A \otimes H$ and $v^{\prime}: A^{\prime} \rightarrow A^{\prime} \otimes H$. Let $A \bigsqcup A^{\prime}$ denote the coproduct of $A$ and $A^{\prime}$ in the category of chain algebras. Since $v$ and $v^{\prime}$ are algebra maps, they together induce an algebra map

$$
\nu^{\prime \prime}: A \coprod A^{\prime} \rightarrow(A \otimes H) \coprod\left(A^{\prime} \otimes H\right) \rightarrow\left(A \coprod A^{\prime}\right) \otimes H,
$$

which satisfies the axioms of a coaction because $v$ and $v^{\prime}$ do. In other words, the algebra coproduct of $H$-comodule algebras is naturally an $H$-comodule algebra. 
It is easy to check that the tensor product $A \otimes A^{\prime}$, with its usual algebra structure, also admits a natural $H$-coaction

$$
A \otimes A^{\prime} \stackrel{v \otimes v^{\prime}}{\longrightarrow}(A \otimes H) \otimes\left(A^{\prime} \otimes H\right) \stackrel{\cong}{\longrightarrow}\left(A \otimes A^{\prime}\right) \otimes(H \otimes H) \stackrel{\operatorname{Id} \otimes \mu}{\longrightarrow}\left(A \otimes A^{\prime}\right) \otimes H
$$

that is an algebra map, where $\mu$ denotes the multiplication in $H$.

Proposition 3.18 Let $(\theta ; \gamma):(H ; A) \rightarrow(K ; B)$ and $\left(\theta ; \gamma^{\prime}\right):\left(H ; A^{\prime}\right) \rightarrow\left(K ; B^{\prime}\right)$ be morphisms in CASH. Endow $A \amalg A^{\prime}$ and $B \otimes B^{\prime}$ with their natural $H$-comodule and $K$-comodule algebra structures. If the algebras underlying $A$ and $A^{\prime}$ are free on free graded $R$-modules $V$ and $V^{\prime}$, respectively, then there exists a morphism

$$
\left(\theta ; \gamma^{\prime \prime}\right):\left(H ; A \coprod A^{\prime}\right) \rightarrow\left(K ; B \otimes B^{\prime}\right)
$$

in CASH such that $\gamma_{0}^{\prime \prime}(v)=\gamma_{0}(v) \otimes 1$ and $\gamma^{\prime \prime}\left(v^{\prime}\right)=1 \otimes \gamma_{0}^{\prime}\left(v^{\prime}\right)$ for all $v \in V$ and $v^{\prime} \in V^{\prime}$.

In the situation of the proposition above, we write

$$
\gamma_{0}^{\prime \prime}=\gamma_{0} \star \gamma_{0}^{\prime}
$$

Proof Let

$$
\xi: \mathcal{T}_{r}(A, H) \underset{\mathcal{A}}{\triangleleft} \mathcal{F} \rightarrow \mathcal{T}(B, K) \quad \text { and } \quad \xi^{\prime}: \mathcal{T}_{r}\left(A^{\prime}, H\right) \underset{\mathcal{A}}{\triangleleft} \mathcal{F} \rightarrow \mathcal{T}\left(B^{\prime}, K\right)
$$

be the morphisms of shifted $\mathcal{A}$-modules of chain complexes, corresponding to $g$ and $g^{\prime}$, with corresponding families $\mathfrak{F}^{+}(\xi)=\left\{\xi_{m} \mid m \geq 0\right\}$ and $\mathfrak{F}^{+}\left(\xi^{\prime}\right)=\left\{\xi_{m}^{\prime} \mid m \geq 0\right\}$.

Define a family of linear maps

$$
\Xi^{\prime \prime}=\left\{\xi_{m}^{\prime \prime}: V \oplus V^{\prime} \rightarrow\left(B \otimes B^{\prime}\right) \otimes K^{\otimes m} \mid m \geq 0\right\}
$$

by $\xi_{m}^{\prime \prime}(v)=\iota_{1} \circ \xi_{m}(v)$ for all $v \in V$ and $\xi_{m}^{\prime \prime}\left(v^{\prime}\right)=\iota_{2} \circ \xi_{m}^{\prime}\left(v^{\prime}\right)$ for all $v^{\prime} \in V^{\prime}$, where

$$
\iota_{1}: B \otimes K^{\otimes m} \rightarrow\left(B \otimes B^{\prime}\right) \otimes K^{\otimes m}: x \otimes y_{1} \otimes \cdots \otimes y_{m} \mapsto x \otimes 1 \otimes y_{1} \otimes \cdots \otimes y_{m}
$$

and

$\iota_{2}: B^{\prime} \otimes K^{\otimes m} \rightarrow\left(B \otimes B^{\prime}\right) \otimes K^{\otimes m}: x^{\prime} \otimes y_{1} \otimes \cdots \otimes y_{m} \mapsto 1 \otimes x^{\prime} \otimes y_{1} \otimes \cdots \otimes y_{m}$.

Note that the algebra map induced by the linear map $\xi_{0}^{\prime \prime}$ is indeed $\gamma_{0} \star \gamma_{0}^{\prime}$.

It is easy to check that the family $\Xi^{\prime \prime}$ satisfies the conditions of Proposition 3.16, since $\xi$ and $\xi^{\prime}$ are morphisms of shifted $\mathcal{A}$-modules of chain complexes. 
Inspired by the sketch of the proof of Theorem 3.13, we can easily verify the following result, establishing extended multiplicative naturality of Cotor, generalizing both Corollary 3.8 and Theorem 3.13.

Proposition 3.19 Let grA denote the category of graded algebras over $R$. The functor Cotor $^{(-)}(-; R)$ of Corollary 3.8 extends to a functor

$$
\operatorname{Cotor}^{(-)}(-; R): \text { CASH } \rightarrow \text { grA. }
$$

Proof Let $H$ and $H^{\prime}$ be simply-connected chain Hopf algebras, and let

$$
\theta \in(\mathcal{A}, \mathcal{F})-\operatorname{PsHopf}\left(H, H^{\prime}\right) .
$$

Let $g: M \rightarrow M^{\prime}$ be a CASH map with respect to $\theta$, where $M$ is a right $H$-comodule, and $M^{\prime}$ is a right $H^{\prime}$-comodule. Recall the graded algebra structure on $\operatorname{Cotor}^{H}(M, R)$ from Corollary 3.8 and its proof.

Let

$$
\xi: \mathcal{T}_{r}(M, H) \underset{\mathcal{A}}{\triangleleft} \mathcal{F} \rightarrow \mathcal{T}\left(M^{\prime}, H^{\prime}\right)
$$

be the morphism of shifted right $\mathcal{A}$-modules corresponding to $g$. Since $g$ is a CASH map, $\operatorname{Ind}^{+}(\xi)$ is a chain algebra map and hence $\operatorname{Cotor}^{\theta}(g, \mathrm{Id})$ is a map of graded algebras.

\section{Path objects and homotopy fibers in F}

In this section we define a functor

$$
\mathfrak{P L}: \mathbf{F} \longrightarrow \mathbf{H},
$$

called the path-loop functor. For every $(C, \Psi)$ in $\mathbf{F}$, there is a natural surjection of chain Hopf algebras $\mathfrak{P} \mathfrak{L}(C, \Psi) \rightarrow \widetilde{\Omega}(C, \Psi)$. The definition of $\mathfrak{P} \mathfrak{L}$ is the first step towards building a particularly nice chain algebra from which we can compute $\operatorname{Cotor}^{H}(R, R)$, when $H$ is a chain Hopf algebra endowed with an Alexander-Whitney model, as defined in Section 5. As we explain in Section 6, the terminology chosen is justified by the fact that the homotopy fiber of the natural surjection $\mathfrak{P} \mathfrak{L} \widetilde{C}(K) \rightarrow \widetilde{\Omega} \widetilde{C}(K)$ is indeed a model for $G^{2} K$, where $\widetilde{C}$ denotes the functor of Theorem 2.13 .

We begin by more general considerations. Given any graded module $X$, let $\bar{X}$ denote $s^{-1} X$, and let $\bar{x}$ denote an element $s^{-1} x$. Let $\sigma: X \rightarrow X \oplus \bar{X}$ be defined by $\sigma(x)=\bar{x}$, and let $\iota: X \rightarrow X \oplus \bar{X}$ denote the inclusion. 
Let $(X, d)$ be any chain complex. The based-path object on $(X, d)$, denoted $\mathfrak{P}(X, d)$, is defined to be the acyclic chain complex $(X \oplus \bar{X}, \widetilde{d})$, where $\widetilde{d} x=\imath d x-\bar{x}$ and $\widetilde{d} \bar{x}=-\overline{d x}, \mathrm{ie}$,

$$
\widetilde{d} \iota=\iota d-\sigma \text { and } \widetilde{d} \sigma=-\sigma d
$$

There is an obvious factorization

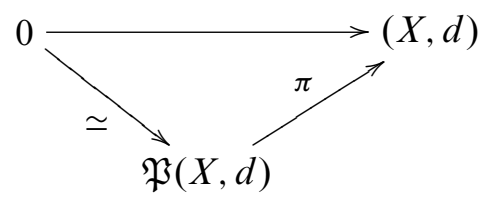

where $\pi$ denotes the obvious projection, justifying the name we have given to the chain complex $\mathfrak{P}(X, d)$.

The based-path construction is clearly natural, ie, there is a functor

$$
\mathfrak{P}: \mathbf{C h}_{R} \rightarrow \mathbf{C h}_{R} \text {. }
$$

Furthermore, the functor $\mathfrak{P}$ is comonoidal, where the natural transformation

$$
\mathrm{j}: \mathfrak{P}(-\otimes-) \rightarrow \mathfrak{P}(-) \otimes \mathfrak{P}(-)
$$

is defined for chain complexes $X$ and $Y$ to be the injection

$\mathfrak{j}_{X, Y}:(X \otimes Y) \oplus \overline{(X \otimes Y)} \cong(X \otimes Y) \oplus(\bar{X} \otimes Y) \oplus(X \otimes \bar{Y}) \hookrightarrow(X \oplus \bar{X}) \otimes(Y \oplus \bar{Y})$.

In particular, if $(X, d, \Delta)$ is a coassociative coalgebra, then $(\mathfrak{P}(X, d), \widetilde{\Delta})$ is also a coassociative coalgebra, where $\widetilde{\Delta}=\mathfrak{j}_{X}, X \mathfrak{P}(\Delta)$. Note that the comultiplication on $\mathfrak{P}(X)$ is specified by $\widetilde{\Delta} \iota=(\iota \otimes \iota) \Delta$ and $\widetilde{\Delta} \sigma=(\sigma \otimes \iota+\iota \otimes \sigma) \Delta$ and that the projection map $\pi: \mathfrak{P}(X) \rightarrow X$ is a morphism of coalgebras.

The morphisms of graded $R$-modules $\iota$ and $\sigma$ induce a morphism of symmetric sequences $\mathcal{D}_{\iota, l}(\sigma): \mathcal{T}(X) \rightarrow \mathcal{T}(\mathfrak{P}(X))$ that is of degree -1 in each level (cf, Definition 1.4), while the differentials $d$ and $\widetilde{d}$ induce $\mathcal{D}(d): \mathcal{T}(X) \rightarrow \mathcal{T}(X)$ and $\mathcal{D}(\widetilde{d}): \mathcal{T}(\mathfrak{P}(X)) \rightarrow \mathcal{T}(\mathfrak{P}(X))$. It is a matter of straightforward calculation to show that (4-1) implies that

$$
\mathcal{D}(\widetilde{d}) \mathcal{D}_{\iota, \iota}(\sigma)=-\mathcal{D}_{\iota, \iota}(\sigma) \mathcal{D}(d) \text { and } \mathcal{D}(\widetilde{d}) \mathcal{T}(\iota)=\mathcal{T}(\iota) \mathcal{D}(d)-\mathcal{D}_{\iota, \iota}(\sigma) .
$$

Proposition 4.1 Let $C$ and $C^{\prime}$ be coassociative chain coalgebras. Any morphism

$$
\theta: \mathcal{T}(C) \diamond_{\mathcal{A}} \mathcal{F} \rightarrow \mathcal{T}\left(C^{\prime}\right)
$$


of right $\mathcal{A}$-modules of chain complexes lifts naturally to a morphism

$$
\widetilde{\theta}: \mathcal{T}(\mathfrak{P}(C)) \diamond_{\mathcal{A}} \mathcal{F} \rightarrow \mathcal{T}\left(\mathfrak{P}\left(C^{\prime}\right)\right),
$$

ie, there is a commuting diagram of morphisms in $(\mathcal{A}, \mathcal{F})$-Coalg

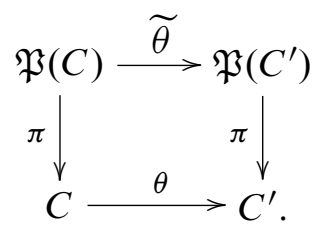

Here, the coalgebra morphisms denoted $\pi$ are considered as morphisms in $(\mathcal{A}, \mathcal{F})$-Coalg via the inclusion functor.

Proof We remark first that since $\theta$ is a differential map, the following equality holds.

$$
\mathcal{D}\left(d^{\prime}\right) \theta=\theta\left(\mathcal{D}(d) \diamond_{\mathcal{A}} 1+1 \diamond_{\mathcal{A}} \partial_{\mathcal{F}}\right) .
$$

Here, the composition rule applied is that of morphisms of right $\mathcal{A}$-modules.

We now define a morphism of symmetric sequences of graded $R$-modules

$$
\theta^{\prime}: \mathcal{L}(C \oplus \bar{C}) \diamond \mathcal{S} \rightarrow \mathcal{T}\left(C^{\prime} \oplus \bar{C}\right)
$$

by $\theta^{\prime}\left(\iota(c) \otimes z_{m-1}\right)=\iota^{\otimes m} \theta\left(c \otimes z_{m-1}\right)$ for all $c \in C$, since $\widetilde{\theta}$ should extend $\theta$, and $\theta^{\prime}\left(\bar{c} \otimes z_{m-1}\right)=\mathcal{D}_{\iota, l}(\sigma) \theta\left(c \otimes z_{m-1}\right)$. In other words,

$$
\theta^{\prime}(\iota \diamond 1)=\mathcal{T}(\iota) \theta \text { and } \theta^{\prime}(\sigma \diamond 1)=\mathcal{D}_{\iota, \iota}(\sigma) \theta
$$

Applying Lemma 2.3, we obtain another morphism of symmetric sequences

$$
\theta^{\prime \prime}: \mathcal{T}(C \oplus \bar{C}) \diamond \mathcal{S} \rightarrow \mathcal{T}\left(C^{\prime} \oplus \bar{C}^{\prime}\right),
$$

defined for all $k$ and for all $w_{1}, \ldots, w_{k} \in C \oplus \bar{C}$ by

$\theta^{\prime \prime}\left(\left(w_{1} \otimes \cdots \otimes w_{k}\right) \otimes\left(z_{n_{1}-1} \otimes \cdots \otimes z_{n_{k}-1}\right)\right)= \pm \theta^{\prime}\left(w_{1} \otimes z_{n_{1}-1}\right) \otimes \cdots \otimes \theta^{\prime}\left(w_{k} \otimes z_{n_{k}-1}\right)$, where the sign is determined by the Koszul rule.

Now use the right $\mathcal{A}$-module structure of $\mathcal{T}\left(\mathfrak{P}\left(C^{\prime}\right)\right)$ to extend $\theta^{\prime \prime}$ to a morphism

$$
\widetilde{\theta}: \mathcal{T}(C \oplus \bar{C}) \diamond_{\mathcal{A}} \mathcal{F} \cong \mathcal{T}(C \oplus \bar{C}) \diamond \mathcal{S} \diamond \mathcal{A} \rightarrow \mathcal{T}\left(C^{\prime} \oplus \bar{C}^{\prime}\right)
$$

of right $\mathcal{A}$-modules of graded $R$-modules. As an easy consequence of (4-4), we have that

$$
\widetilde{\theta}\left(\mathcal{T}(\iota) \diamond_{\mathcal{A}} 1\right)=\mathcal{T}(\iota) \theta \text { and } \widetilde{\theta}\left(\mathcal{D}_{\iota, \iota}(\sigma)\right)=\mathcal{D}_{\iota, \iota}(\sigma) \theta
$$


To complete the proof, we need to verify that $\widetilde{\theta}$ is differential, ie, that

$$
\widetilde{\theta}\left(\mathcal{D}(d) \diamond_{\mathcal{A}} 1+1 \diamond_{\mathcal{A}} \partial_{\mathcal{F}}\right)=\mathcal{D}\left(\widetilde{d}^{\prime}\right) \widetilde{\theta}
$$

It is enough to prove that the two sides of the equation are equal when precomposed (as maps of right $\mathcal{A}$-modules) with either $\mathcal{L}(\iota) \diamond 1: \mathcal{L}(C) \diamond \mathcal{F} \rightarrow \mathcal{T}(\mathfrak{P}(C)) \diamond_{\mathcal{A}} \mathcal{F}$ or $\mathcal{L}(\sigma) \diamond 1: \mathcal{L}(C) \diamond \mathcal{F} \rightarrow \mathcal{T}(\mathfrak{P}(C)) \diamond{ }_{\mathcal{A}} \mathcal{F}$.

Observe that

$$
\begin{aligned}
\mathcal{D}(\widetilde{d}) \widetilde{\theta}(\mathcal{L}(\iota) \diamond 1) & =\mathcal{D}(\widetilde{d}) \mathcal{T}(\iota) \theta \\
& =\mathcal{D}_{\iota, \iota}(\widetilde{d} \iota) \theta \\
& =\mathcal{D}_{\iota, \iota}(\iota d-\sigma) \theta \\
& =\left(\mathcal{T}(\iota) \mathcal{D}\left(d^{\prime}\right)-\mathcal{D}_{\iota, \iota}(\sigma)\right) \theta,
\end{aligned}
$$

while

$$
\begin{aligned}
\widetilde{\theta}\left(\mathcal{D}(\widetilde{d}) \diamond_{\mathcal{A}} 1\right)(\mathcal{L}(\iota) \diamond 1) & =\widetilde{\theta}\left(\mathcal{T}(\iota) \mathcal{D}(d) \diamond_{\mathcal{A}} 1-\mathcal{D}_{\iota, \iota}(\sigma) \diamond_{\mathcal{A}} 1\right) \\
& =\mathcal{T}(\iota) \theta \mathcal{D}(d)-\widetilde{\theta}\left(\mathcal{D}_{\iota, \iota}(\sigma) \diamond_{\mathcal{A}} 1\right) .
\end{aligned}
$$

Thus,

$$
\begin{aligned}
\left(\mathcal{D}(\widetilde{d}) \widetilde{\theta}-\widetilde{\theta}\left(\mathcal{D}(\widetilde{d}) \diamond_{\mathcal{A}} 1\right)\right)(\mathcal{L}(\iota) \diamond 1) & =\mathcal{T}(\iota)\left(\mathcal{D}\left(d^{\prime}\right) \theta-\theta \mathcal{D}(d)\right) \\
& =\mathcal{T}(\iota) \theta\left(1 \diamond_{\mathcal{A}} \partial_{\mathcal{F}}\right) \\
& =\widetilde{\theta}\left(1 \diamond_{\mathcal{A}} \partial_{\mathcal{F}}\right)(\mathcal{L}(\iota) \diamond 1),
\end{aligned}
$$

by equation (4-3).

Similarly,

$$
\mathcal{D}\left(\widetilde{d}^{\prime}\right) \widetilde{\theta}(\mathcal{L}(\sigma) \diamond 1)=\mathcal{D}\left(\widetilde{d}^{\prime}\right) \mathcal{D}_{\iota, \iota}(\sigma) \theta=-\mathcal{D}_{\iota, \iota}(\sigma) \mathcal{D}\left(d^{\prime}\right) \theta
$$

and

$$
\widetilde{\theta}\left(\mathcal{D}(\widetilde{d}) \diamond_{\mathcal{A}} 1\right)(\mathcal{L}(\sigma) \diamond 1)=-\widetilde{\theta}\left(\mathcal{D}_{\iota, \iota}(\sigma) \mathcal{D}(d) \diamond_{\mathcal{A}} 1\right)=-\left(\mathcal{D}_{\iota, l}(\sigma) \theta\left(\mathcal{D}(d) \diamond_{\mathcal{A}} 1\right) .\right.
$$

Thus,

$$
\begin{aligned}
\left(\mathcal{D}\left(\widetilde{d}^{\prime}\right) \widetilde{\theta}-\widetilde{\theta}\left(\mathcal{D}(\widetilde{d}) \diamond_{\mathcal{A}} 1\right)\right)(\mathcal{L}(\sigma) \diamond 1) & =-\left(\mathcal{D}_{\iota, l}(\sigma) \theta\left(1 \diamond_{\mathcal{A}} \partial_{\mathcal{F}}\right)\right. \\
& =-\widetilde{\theta}\left(\mathcal{D}_{\iota, l}(\sigma) \diamond_{\mathcal{A}} 1\right)\left(1 \diamond_{\mathcal{A}} \partial_{\mathcal{F}}\right) \\
& =\widetilde{\theta}\left(1 \diamond_{\mathcal{A}} \partial_{\mathcal{F}}\right)(\mathcal{L}(\sigma) \diamond 1),
\end{aligned}
$$

again by (4-3). 
Recall that $\mathfrak{I}_{\mathcal{F}}: \mathcal{A}$-Coalg $\rightarrow(\mathcal{A}, \mathcal{F})$-Coalg denotes the "inclusion" functor (1-1).

Corollary 4.2 If $(C, \Psi)$ is an object in $\mathbf{F}$, then the based-path object $\mathfrak{P}(C)$ admits a natural Alexander-Whitney coalgebra structure map $\widetilde{\Psi}$, extending $\Psi$. Furthermore, the morphism of right $\mathcal{A}$-modules

$$
\mathfrak{I}_{\mathcal{F}}(\pi)=\mathcal{T}(\pi) \diamond_{\mathcal{A}} \varepsilon: \mathcal{T}(\mathfrak{P}(C)) \diamond_{\mathcal{A}} \mathcal{F} \rightarrow \mathcal{T}(C)
$$

induced by the natural projection map of chain complexes $\pi: \mathfrak{P}(C) \rightarrow C$ is a morphism in $\mathbf{F}$, ie,

$$
\Psi \mathfrak{I}_{\mathcal{F}}(\pi)=\left(\mathfrak{I}_{\mathcal{F}}(\pi) \wedge \mathfrak{I}_{\mathcal{F}}(\pi)\right) \widetilde{\Psi}
$$

where the composition is calculated in $(\mathcal{A}, \mathcal{F})$-Coalg.

Proof By Proposition 4.1, the morphism of right $\mathcal{A}$-modules

$$
\Psi: \mathcal{T}(C) \diamond_{\mathcal{A}} \mathcal{F} \rightarrow \mathcal{T}(C \otimes C)
$$

gives rise naturally to

$$
\widetilde{\Psi}: \mathcal{T}(\mathfrak{P}(C)) \diamond_{\mathcal{A}} \mathcal{F} \rightarrow \mathcal{T}(\mathfrak{P}(C \otimes C)) .
$$

Since $\mathfrak{P}(C \otimes C)$ injects into $\mathfrak{P}(C) \otimes \mathfrak{P}(C)$, we can look at $\widetilde{\Psi}$ as a morphism with target $\mathcal{T}(\mathfrak{P}(C) \otimes \mathfrak{P}(C))$.

To complete the proof that $(\mathfrak{P}(C), \widetilde{\Psi})$ is an Alexander-Whitney coalgebra, we need to check that $q \operatorname{Ind}(\widetilde{\Psi})$ is coassociative. By naturality, however, this follows immediately from the coassociativity of $q \operatorname{Ind}(\Psi)$.

Verification that $\mathfrak{I}_{\mathcal{F}}(\pi)$ is a morphism in $\mathbf{F}$ is trivial.

Proposition 4.3 Let $\theta:(C, \Psi) \rightarrow\left(C^{\prime}, \Psi^{\prime}\right)$ be a morphism in $\mathbf{F}$. Then

$$
\widetilde{\theta}:(\mathfrak{P}(C), \widetilde{\Psi}) \rightarrow\left(\mathfrak{P}\left(C^{\prime}\right), \widetilde{\Psi}^{\prime}\right)
$$

is also a morphism in $\mathbf{F}$, ie, $(\widetilde{\theta} \wedge \widetilde{\theta}) \widetilde{\Psi}=\widetilde{\Psi}^{\prime} \widetilde{\theta}$, where the composition is performed in $(\mathcal{A}, \mathcal{F})$-Coalg.

Proof It suffices to check the desired equality holds when precomposed (as morphisms of right $\mathcal{A}$-modules) with either $\mathcal{L}(\iota) \diamond 1$ or $\mathcal{L}(\sigma) \diamond 1$. To distinguish between composition as morphisms of right $\mathcal{A}$-modules and as morphisms in $(\mathcal{A}, \mathcal{F})$-Coalg, we denote the first by simple concatentation of symbols and the second by $\circ$. 
The definition of $\widetilde{\Psi}$ given in the proofs of Proposition 4.1 and Corollary 4.2 implies that

$$
\begin{aligned}
(\widetilde{\theta} \wedge \tilde{\theta}) \circ \widetilde{\Psi}(\mathcal{L}(\iota) \diamond 1) & =(\widetilde{\theta} \curlywedge \widetilde{\theta}) \circ \mathcal{T}(\iota) \Psi \\
& =(\widetilde{\theta} \mathcal{T}(\iota) \curlywedge \widetilde{\theta} \mathcal{T}(\iota)) \circ \Psi \\
& =\mathcal{T}(\iota)(\theta \curlywedge \theta) \circ \Psi \\
& =\mathcal{T}(\iota) \Psi^{\prime} \circ \theta \text { since } \theta \text { is a morphism in } \mathbf{F} \\
& =\widetilde{\Psi}^{\prime} \circ \mathcal{T}(\iota) \theta \\
& =\widetilde{\Psi}^{\prime} \widetilde{\theta}(\mathcal{L}(\iota) \diamond 1) .
\end{aligned}
$$

On the other hand,

$$
\begin{aligned}
(\widetilde{\theta} \wedge \widetilde{\theta}) \circ \widetilde{\Psi}(\mathcal{L}(\sigma) \diamond 1) & =(\widetilde{\theta} \wedge \widetilde{\theta}) \circ \mathcal{D}_{\iota, l}(\sigma) \Psi \\
& =\left(\widetilde{\theta} \mathcal{D}_{\iota, \iota}(\sigma) \curlywedge \widetilde{\theta} \mathcal{T}(\iota)+\widetilde{\theta} \mathcal{T}(\iota) \curlywedge \widetilde{\theta} \mathcal{D}_{\iota, l}(\sigma)\right) \circ \Psi \\
& =\mathcal{D}_{\iota, l}(\sigma)(\theta \curlywedge \theta) \circ \Psi \\
& =\mathcal{D}_{\iota, \iota}(\sigma) \Psi^{\prime} \circ \theta \text { since } \theta \text { is a morphism in } \mathbf{F} \\
& =\widetilde{\Psi}^{\prime} \circ \mathcal{D}_{\iota, \iota}(\sigma) \theta \\
& =\widetilde{\Psi}^{\prime} \widetilde{\theta}(\mathcal{L}(\sigma) \diamond 1) .
\end{aligned}
$$

This completes the proof.

Corollary 4.2 and Proposition 4.3 imply that the following definition makes sense.

Definition 4.4 The based-path functor $\widetilde{\mathfrak{P}}: \mathbf{F} \rightarrow \mathbf{F}$ is defined on objects by $\widetilde{\mathfrak{P}}(C, \Psi)=$ $(\mathfrak{P}(C), \widetilde{\Psi})$ and on morphisms by $\widetilde{\mathfrak{P}}(\theta)=\widetilde{\theta}$.

The second part of Corollary 4.2 implies that $\widetilde{\mathfrak{P}}$ is augmented: the projection $\pi$ serves as a natural transformation $\pi: \widetilde{\mathfrak{P}} \rightarrow \operatorname{Id}_{\mathbf{F}}$.

For the constructions in the following sections, we need a relative version of the path functor, ie, a notion of homotopy fiber in $\mathbf{F}$. We consider first the notion of homotopy fiber in $\mathbf{C h}_{R}$. Any morphism of chain complexes $f: X \rightarrow Y$ can be factored as

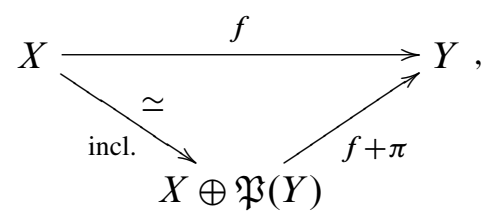

Algebraic 83 Geometric Topology, Volume 7 (2007) 
so that it is reasonable to define the homotopy fiber $\mathfrak{H} \mathfrak{F}(f)$ to be the pullback

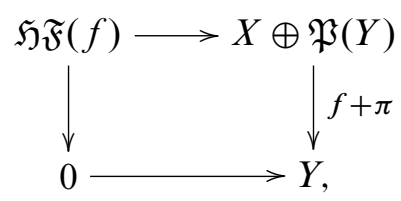

or, more prosaically, $\mathfrak{H} \mathfrak{F}(f)$ is the kernel of $f+\pi$.

Analogously, to define homotopy fibers in $\mathbf{F}$, we first need to show that $\mathbf{F}$ admits coproducts. It is easy to see, however, that if $(C, \Psi)$ and $\left(C^{\prime}, \Psi^{\prime}\right)$ are AlexanderWhitney coalgebras, then their coproduct $(C, \Psi) \amalg\left(C^{\prime}, \Psi^{\prime}\right)$ in $\mathbf{F}$ is $\left(C \oplus C^{\prime}, \Psi^{\prime \prime}\right)$, where

$$
\Psi^{\prime \prime}: \mathcal{T}\left(C \oplus C^{\prime}\right) \diamond_{\mathcal{A}} \mathcal{F} \rightarrow \mathcal{T}\left(\left(C \oplus C^{\prime}\right)^{\otimes 2}\right)
$$

is the morphism of $\mathcal{A}$-modules specified (as in the proofs of Lemma 2.3 and Proposition 4.1) by

$$
\Psi^{\prime \prime}\left(c \otimes z_{k-1}\right)=\Psi\left(c \otimes z_{k-1}\right) \text { and } \Psi^{\prime \prime}\left(c^{\prime} \otimes z_{k-1}\right)=\Psi^{\prime}\left(c^{\prime} \otimes z_{k-1}\right)
$$

for all $c \in C, c^{\prime} \in C^{\prime}$ and $k>0$.

Let $\theta:\left(C^{\prime}, \Psi^{\prime}\right) \rightarrow(C, \Psi)$ be a morphism in $\mathbf{F}$. There is an obvious factorization in $\mathbf{F}$

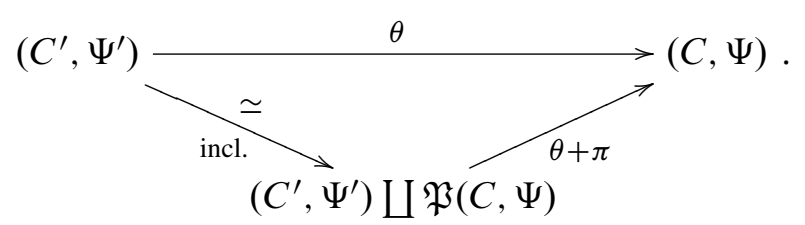

Note that $\theta+\pi$, seen simply as a map of coalgebras, admits a (nondifferential) section, the coalgebra map

$$
\text { j: } C \rightarrow C^{\prime} \oplus(C \oplus \bar{C}),
$$

which is just the natural inclusion. Moreover, $\Psi^{\prime \prime}\left(j(c) \otimes z_{k-1}\right)=j^{\otimes k} \Psi\left(c \otimes z_{k-1}\right)$ for all $k$, so that the induced algebra map

$$
\Omega \mathrm{j}: \Omega C \rightarrow \Omega\left(C^{\prime} \oplus(C \oplus \bar{C})\right)
$$

commutes with the induced comultiplications, ie, $\Omega \mathrm{j}$ is a Hopf algebra map, which is a (nondifferential) section of $\Omega(\theta+\pi)$.

Recall the functor $\widetilde{\Omega}: \mathbf{F} \rightarrow \mathbf{H}$ from (2-3).

Definition 4.5 The algebraic path-loop functor

$$
\mathfrak{P} \mathfrak{L}: \mathbf{F} \longrightarrow \mathbf{H}
$$

Algebraic $8 \mathcal{G}$ Geometric Topology, Volume 7 (2007) 
is the composite $\mathfrak{P} \mathfrak{L}=\widetilde{\Omega} \circ \widetilde{\mathfrak{P}}$. The induced comultiplication on $\mathfrak{P} \mathfrak{L}(C, \Psi)$ is denoted $\widetilde{\psi}$.

We prefer the notation $\mathfrak{P} \mathfrak{L}$ for this functor, instead of $\widetilde{\Omega} \circ \widetilde{\mathfrak{P}}$, as it reminds us that $\mathfrak{P} \mathfrak{L}(C, \Psi)$ plays the role of the paths on a loop space.

Observe that $\mathfrak{P} \mathfrak{L}(C)$ is always acyclic, since $\widetilde{\mathfrak{P}}(C)$ is acyclic and $1-$ connected.

Consider the natural right $\Omega C$-comodule structure on $\mathfrak{P} \mathfrak{L}(C)$ given by the coaction

$$
v=(1 \otimes \Omega \pi) \widetilde{\psi}: \Omega(C \oplus \bar{C}) \rightarrow \Omega(C \oplus \bar{C}) \otimes \Omega C .
$$

It is important for the proof of Theorem 5.6 to know that $\mathfrak{P} \mathfrak{L}(C)$ is a cofree right $\Omega C$-comodule, which is an immediate consequence of the following more general result.

Proposition 4.6 Let $p:\left(H^{\prime}, d^{\prime}\right) \rightarrow(H, d)$ be a surjection of connected chain Hopf algebras, which are free as graded $R$-modules. Let $v=(1 \otimes p) \Delta^{\prime}: H^{\prime} \rightarrow H^{\prime} \otimes H$ denote the right $H$-coaction on $H^{\prime}$ induced by $p$. If $p$ admits a (nondifferential) Hopf algebra section $s$, then

(1) $H^{\prime} \square_{H} R$ is a sub chain algebra of $H^{\prime}$, and

(2) $\left(H^{\prime}, v\right)$ is cofree as a nondifferential $H$-comodule, with cobasis $H^{\prime} \square_{H} R$.

Proof Let $\left(B, d_{B}\right)=H^{\prime} \square_{H} R$. Milnor-Moore [13, Theorem 4.6], implies directly that $H^{\prime} \square_{H} R$ is a sub chain algebra of $H^{\prime}$.

Consider the linear map

$$
h=\mu(i \otimes s): B \otimes H \stackrel{\cong}{\longrightarrow} H^{\prime},
$$

where $\mu$ denotes the multiplication on $H^{\prime}$ and $i$ is the canonical inclusion. According to [13, Theorem 4.7], $h$ is an isomorphism of both right $H$-comodules and left $B-$ modules, since the underlying Hopf algebra of $H$ is connected, while the underlying algebra of $H^{\prime}$ is a connected $H$-comodule algebra, and all graded $R$-modules in question are free. Since $h$ is an isomorphism, we can use it to define a differential $\bar{d}$ on $B \otimes H$ by

$$
\bar{d}=h^{-1} d^{\prime} h .
$$

Then $h$ becomes an isomorphism of differential right $H$-comodules and left $B-$ modules, ie, $H^{\prime}$ is cofree. 
Observe that for all $x \in B$,

$$
\begin{aligned}
\bar{d}(x \otimes 1) & =h^{-1} d^{\prime}(i(x)) \\
& =h^{-1} i\left(d_{B} x\right) \\
& =d_{B} x \otimes 1,
\end{aligned}
$$

ie, the restriction of $\bar{d}$ to $B \otimes 1$ is simply $d_{B} \otimes 1$. In other words, the inclusion of $B$ into $H^{\prime}$ is a differential map.

Corollary 4.7 The path-loop construction $\mathfrak{P} \mathfrak{L}(C, \Psi)$ on any Alexander-Whitney coalgebra $(C, \Psi)$ is cofree over $\widetilde{\Omega}(C, \Psi)$, with cobasis $\mathfrak{P} \mathfrak{L}(C, \Psi) \square \widetilde{\Omega}_{(C, \Psi)} R$, which is a sub chain algebra of $\mathfrak{P} \mathfrak{L}(C, \Psi)$.

More generally, for any $\theta \in \mathbf{F}\left(\left(C^{\prime}, \Psi^{\prime}\right),(C, \Psi)\right)$, the coalgebra $\widetilde{\Omega}\left(\left(C^{\prime}, \Psi^{\prime}\right) \amalg \mathfrak{P}(C, \Psi)\right)$ is cofree over $\widetilde{\Omega}(C, \Psi)$, with cobasis $\widetilde{\Omega}\left(\left(C^{\prime}, \Psi^{\prime}\right) \amalg \mathfrak{P}(C, \Psi)\right) \square \widetilde{\Omega}_{(C, \Psi)} R$, which is a sub chain algebra of $\widetilde{\Omega}\left(\left(C^{\prime}, \Psi^{\prime}\right) \bigsqcup \mathfrak{P}(C, \Psi)\right)$.

Proof Since $\widetilde{\Omega}(\theta+\pi): \widetilde{\Omega}\left(\left(C^{\prime}, \Psi^{\prime}\right) \bigsqcup \mathfrak{P}(C, \Psi)\right) \rightarrow \widetilde{\Omega}(C, \Psi)$ admits a (nondifferential) Hopf algebra section $\Omega$ j, we can apply Proposition 4.6.

To conclude this section we analyze more precisely the nature of $\widetilde{\psi}$, the comultiplication on $\mathfrak{P} \mathfrak{L}(C)$, and $v$, the induced $\widetilde{\Omega} C$-coaction. Let $\iota$ denote the natural (nondifferential) section $\Omega C \hookrightarrow \Omega(C \oplus \bar{C})$ of $\Omega \pi$. Let

$$
\kappa: \Omega C \rightarrow \Omega(C \oplus \bar{C})
$$

denote the $(\iota, \iota)$-derivation of degree -1 specified by $\kappa\left(s^{-1} c\right)=-s^{-1} \bar{c}$, ie, $\kappa \mu=$ $\mu(\kappa \otimes \iota+\iota \otimes \kappa)$.

Lemma 4.8 The derivation $\kappa$ satisfies the following properties.

(1) $\kappa$ is a differential map of degree -1 , ie, $\kappa d_{\Omega}=-\widetilde{d}_{\Omega} \kappa$.

(2) $\kappa$ is a $(\iota, \iota)$-coderivation, ie,

$$
\widetilde{\psi} \kappa=(\kappa \otimes \iota+\iota \otimes \kappa) \psi: \Omega C \rightarrow \Omega(C \oplus \bar{C}) \otimes \Omega(C \oplus \bar{C}) .
$$

(3) $\kappa$ is a map of right $\Omega C$-comodules, ie, $v \kappa=(\kappa \otimes 1) \psi: \Omega C \rightarrow \Omega(C \oplus \bar{C}) \otimes \Omega C$.

Proof (1) Let $c \in C$, and let $c_{i} \otimes c^{i}$ denote its reduced comultiplication. Then, using the definitions of $\widetilde{d}$ and $\widetilde{\Delta}$ from the beginning of Section 4 as well as the definition 
of the cobar construction differential from the introduction, we obtain

$$
\begin{aligned}
\kappa d_{\Omega}\left(s^{-1} c\right) & =\kappa\left(-s^{-1} d c+(-1)^{c_{i}} \cdot s^{-1} c_{i} s^{-1} c^{i}\right) \\
& =s^{-1} \overline{d c}+(-1)^{c_{i}}\left(-s^{-1} \bar{c}_{i} \cdot s^{-1} c^{i}-(-1)^{c_{i}} s^{-1} c_{i} \cdot s^{-1} \bar{c}^{i}\right) \\
& =\widetilde{d}_{\Omega} s^{-1} \bar{c} \\
& =-\widetilde{d}_{\Omega} \kappa\left(s^{-1} c\right) .
\end{aligned}
$$

(2) We have defined $\widetilde{\psi}$ so that the desired equality obviously holds on the generators $s^{-1} C_{+}$. Thus, to establish that the equality holds on all of $\Omega C$, we must show that

$$
\widetilde{\psi} \kappa \mu=(\kappa \otimes \iota+\iota \otimes \kappa) \psi \mu: \Omega C^{\otimes 2} \rightarrow \Omega(C \oplus \bar{C})^{\otimes 2}
$$

where $\mu$ denotes the multiplication map.

We verify this equality by induction on total length of elements in $\Omega C^{\otimes 2}$. By definition of $\widetilde{\psi}$, the equality holds for total length equal to 1 . Suppose that it holds for all elements of $\Omega C^{\otimes 2}$ of total length less than $n$.

Let $\tau$ denote the usual twisting isomorphism $\tau: A \otimes B \cong B \otimes A$. Observe that on $T^{m} s^{-1} C_{+} \otimes T^{n-m} S^{-1} C_{+}$,

$$
\begin{aligned}
\widetilde{\psi} \kappa \mu & =\widetilde{\psi} \mu(\kappa \otimes \imath+\iota \otimes \kappa) \\
& =(\mu \otimes \mu)(1 \otimes \tau \otimes 1)(\widetilde{\psi} \otimes \widetilde{\psi})(\kappa \otimes \iota+\iota \otimes \kappa) \\
& =(\mu \otimes \mu)(1 \otimes \tau \otimes 1)(((\kappa \otimes \iota+\iota \otimes \kappa) \psi) \otimes \psi+\psi \otimes((\kappa \otimes \iota+\iota \otimes \kappa) \psi)) \\
& =(\mu \otimes \mu)\left(\kappa \otimes \iota^{\otimes 3}+\iota^{\otimes 2} \otimes \kappa \otimes \iota+\iota \otimes \kappa \otimes \iota^{\otimes 2}+\iota^{\otimes 3} \otimes \kappa\right)(1 \otimes \tau \otimes 1)(\psi \otimes \psi) \\
& =((\mu(\kappa \otimes \iota+\iota \otimes \kappa)) \otimes \mu+\mu \otimes(\mu(\kappa \otimes \iota+\iota \otimes \kappa)))(1 \otimes \tau \otimes 1)(\psi \otimes \psi) \\
& =(\kappa \mu \otimes \mu+\mu \otimes \kappa \mu)(1 \otimes \tau \otimes 1)(\psi \otimes \psi) \\
& =(\kappa \otimes \iota+\iota \otimes \kappa) \psi \mu .
\end{aligned}
$$

The induction hypothesis assures that the third equality in this sequence holds.

The equality of part (2) of the lemma therefore holds for all elements of total length $n$.

(3) This is an immediate consequence of (2).

\section{Homology of homotopy fibers in $\mathrm{H}$}

In this section we describe the homology of the homotopy fiber $\mathfrak{L}_{2}(C, \Psi)$ of the path-loop map $\Omega \pi: \mathfrak{P} \mathfrak{L}(C, \Psi) \rightarrow \widetilde{\Omega}(C, \Psi)$ on an object $(C, \Psi)$ of $\mathbf{F}$. We show in 
particular that when a chain Hopf algebra $H$ is endowed with an Alexander-Whitney model $\theta: \widetilde{\Omega}(C, \Psi) \rightarrow H$ (see below), then

$$
\mathrm{H}_{*}\left(\mathfrak{L}_{2}(C, \Psi)\right) \cong \operatorname{Cotor}^{H}(R, R)
$$

as graded algebras. We emphasize that this is a true isomorphism and not merely an isomorphism of associated bigraded complexes: there are no extension problems to solve. More generally, we apply the path-loop construction to building a model for computation of the algebra structure of $\operatorname{Cotor}^{H}\left(H^{\prime}, R\right)$, the homology of the homotopy fiber of a map of chain Hopf algebras $H^{\prime} \rightarrow H$, which endows $H^{\prime}$ with the structure of an $H$-comodule algebra. In Section 6 we show that our terminology is fully justified by its application to chain complexes of simplicial sets.

The chain Hopf algebras that we can study by the methods of this paper possess a model of the following sort. We use here the notion of pseudo $\mathcal{A}$-Hopf algebras of Definition 2.2.

Definition 5.1 Let $H$ be a chain Hopf algebra, seen as a pseudo $\mathcal{A}$-Hopf algebra, via the "inclusion" functor $\mathfrak{I}_{\mathcal{F}}: \mathbf{H} \rightarrow(\mathcal{A}, \mathcal{F})$-PsHopf. An Alexander-Whitney model of $H$ consists of an object $(C, \Psi)$ of $\mathbf{F}$ together with a morphism

$$
\Theta \in(\mathcal{A}, \mathcal{F})-\operatorname{PsHopf}(\widetilde{\Omega}(C, \Psi), H)
$$

restricting to a quasi-isomorphism of chain algebras

$$
\theta=\Theta\left(-\otimes z_{0}\right): \widetilde{\Omega}(C, \Psi) \stackrel{\simeq}{\longrightarrow} H .
$$

Unrolling the definition, we see that the existence of $\Theta$ is equivalent to the existence of a family of $R$-linear maps

$$
\mathfrak{F}(\Theta)=\left\{\theta_{n}=\Theta\left(-\otimes z_{n}\right): \widetilde{\Omega}(C, \Psi) \rightarrow H^{\otimes n+1}\right\}
$$

satisfying certain conditions with respect to the differentials (cf, (1-5) and Proposition 2.5). Furthermore, it follows from the formula for the level comultiplication in $\mathcal{F}$ (cf [8, page 854]) that for all $a, b \in \widetilde{\Omega}(C, \Psi)$,

$\theta_{n}(a b)=\sum_{\substack{1 \leq k \leq n+1 \\ \vec{i} \in I_{k, n+1}}} \pm\left(\left(\Delta^{\left(i_{1}\right)} \otimes \cdots \otimes \Delta^{\left(i_{k}\right)}\right) \theta_{k-1}(a)\right) \bullet\left(\left(\theta_{i_{1}-1} \otimes \cdots \otimes \theta_{i_{k}-1}\right) \Delta^{(k)}(b)\right)$,

where $\bullet$ denotes the multiplication in $H^{\otimes n+1}, \Delta$ denotes the comultiplication in $\widetilde{\Omega}(C, \Psi)$ and in $H$ and the signs follow from the Koszul rule. 
Throughout this section we assume that any chain Hopf algebra mentioned is endowed with an Alexander-Whitney model, which we usually denote simply by $\theta: \widetilde{\Omega}(C, \Psi) \rightarrow$ $H$.

Let $H$ be a chain Hopf algebra, and consider the acyclic $H$-comodule algebra $\Omega H \otimes_{t_{\Omega}}$ $H$, as constructed in Proposition 3.5. Let

$$
\mathfrak{p}: \Omega H \otimes_{t_{\Omega}} H \longrightarrow H
$$

denote the natural projection.

We explain first how to lift $\theta \circ \widetilde{\Omega} \pi$ naturally to a quasi-isomorphism $\widetilde{\theta}: \mathfrak{P} \mathfrak{L}(C, \Psi) \rightarrow$ $\Omega H \otimes_{t_{\Omega}} H$ such that the following square commutes.

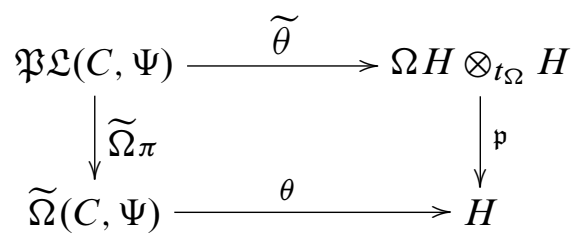

We begin by defining and studying a certain section of $\mathfrak{p}$ and a derivation homotopy associated with it. Let $\chi: H \longrightarrow H \otimes H$ denote the comultiplication on $H$.

The proof of the following lemma is an immediate consequence of the definitions.

Lemma 5.2 Define $\mathfrak{s :} H \rightarrow \Omega H \otimes_{t_{\Omega}} H$ to be the linear map of degree 0 given by $\mathfrak{s}(w)=1 \otimes w$. Then

(1) $\mathfrak{p s}=1_{H}$;

(2) $\left(D_{\Omega} \mathfrak{s}-\mathfrak{s} d\right)(w)=-s^{-1} w_{i} \otimes w^{i}$ for all $w \in H$, where $\bar{\chi}(w)=w_{i} \otimes w^{i}$;

(3) $\mathfrak{s}$ is a map of graded algebras; and

(4) $\mathfrak{s}$ is a map of right $H$-comodules.

Using this knowledge of $\mathfrak{s}$, we can build an important chain map from $H$ to $\Omega H \otimes_{t_{\Omega}} H$, as explained in the next lemma.

Lemma 5.3 Let $\mathfrak{h}=D_{\Omega} \mathfrak{s}-\mathfrak{s} d: H \rightarrow \Omega H \otimes_{t_{\Omega}} H$. Then

(1) $\mathfrak{p h}=0$;

(2) $\mathfrak{h}$ is a chain map of degree -1 , ie, $D_{\Omega} \mathfrak{h}=-\mathfrak{h} d$;

(3) $\mathfrak{h}(a \cdot b)=\mathfrak{h}(a) \cdot \mathfrak{s}(b)+(-1)^{a} \mathfrak{s}(a) \cdot \mathfrak{h}(b)$ for all $a, b \in H$, ie, $\mathfrak{h}$ is a derivation homotopy from $\mathfrak{s}$ to itself; and 
(4) $\mathfrak{h}$ is a map of right $H$-comodules.

Proof We leave the trivial verifications of (1) and (2) to the reader.

(3) Observe that

$$
\begin{aligned}
\mathfrak{h}(a \cdot b) & =D_{\Omega} \mathfrak{s}(a \cdot b)-\mathfrak{s} d(a \cdot b) \\
& =D_{\Omega}(\mathfrak{s}(a) \cdot \mathfrak{s}(b))-\mathfrak{s}\left(d a \cdot b+(-1)^{a} a \cdot d b\right) \\
& =D_{\Omega} \mathfrak{s}(a) \cdot \mathfrak{s}(b)+(-1)^{a} \mathfrak{s}(a) \cdot D_{\Omega} \mathfrak{s}(b)-\mathfrak{s} d(a) \cdot \mathfrak{s}(b)-(-1)^{a} \mathfrak{s}(a) \cdot \mathfrak{s} d(b) \\
& =\mathfrak{h}(a) \cdot \mathfrak{s}(b)+(-1)^{a} \mathfrak{s}(a) \cdot \mathfrak{h}(b) .
\end{aligned}
$$

(4) Observe that

$$
\begin{aligned}
(1 \otimes \chi) \mathfrak{h} & =(1 \otimes \chi)\left(D_{\Omega} \mathfrak{s}-\mathfrak{s} d\right) \\
& =\left(D_{\Omega} \otimes 1+1 \otimes d\right)(1 \otimes \chi) \mathfrak{s}-(\mathfrak{s} \otimes 1) \chi d \\
& =\left(D_{\Omega} \otimes 1+1 \otimes d\right)(\mathfrak{s} \otimes 1) \chi-(\mathfrak{s} \otimes 1)(d \otimes 1+1 \otimes d) \chi \\
& =(\mathfrak{h} \otimes 1) \chi .
\end{aligned}
$$

This completes the proof.

We now apply $\mathfrak{s}$ and $\mathfrak{h}$ to the construction of the lift of $\widetilde{\theta}$.

Theorem 5.4 Let $\widetilde{\theta}: T s^{-1}(C \oplus \bar{C})_{+} \rightarrow \Omega H \otimes_{t_{\Omega}} H$ be the graded algebra map specified by $\widetilde{\theta}\left(s^{-1} c\right)=\mathfrak{s} \theta\left(s^{-1} c\right)$ and $\widetilde{\theta}\left(s^{-1} \bar{c}\right)=\mathfrak{h} \theta\left(s^{-1} c\right)$.

(1) $\widetilde{\theta}$ is a differential map, ie, $\widetilde{\theta} \widetilde{d}_{\Omega}=D_{\Omega} \widetilde{\theta}$, and is therefore a quasi-isomorphism.

(2) $\widetilde{\theta}: \mathfrak{P} \mathfrak{L}(C) \rightarrow \Omega H \otimes_{t_{\Omega}} H$ is a CASH map.

Proof (1) Let $c \in C$ and write $\bar{\Delta}(c)=c_{i} \otimes c^{i}$. Then

$$
\begin{aligned}
D_{\Omega} \widetilde{\theta}\left(s^{-1} c\right) & =D_{\Omega} \mathfrak{s} \theta\left(s^{-1} c\right) \\
& =\mathfrak{s} d \theta\left(s^{-1} c\right)+\mathfrak{h} \theta\left(s^{-1} c\right) \\
& =\mathfrak{s} \theta d_{\Omega}\left(s^{-1} c\right)+\widetilde{\theta}\left(s^{-1} \bar{c}\right) \\
& =\mathfrak{s} \theta\left(-s^{-1}(d c)+(-1)^{c_{i}} s^{-1} c_{i} s^{-1} c^{i}\right)+\widetilde{\theta}\left(s^{-1} \bar{c}\right) \\
& =-\widetilde{\theta}\left(s^{-1}(d c)\right)+(-1)^{c_{i}} \mathfrak{s} \theta\left(s^{-1} c_{i}\right) \mathfrak{s} \theta\left(s^{-1} c^{i}\right)+\widetilde{\theta}\left(s^{-1} \bar{c}\right) \\
& =-\widetilde{\theta}\left(s^{-1}(d c)\right)+(-1)^{c_{i}} \widetilde{\theta}\left(s^{-1} c_{i}\right) \widetilde{\theta}\left(s^{-1} c^{i}\right)+\widetilde{\theta}\left(s^{-1} \bar{c}\right) \\
& =\widetilde{\theta}\left(-s^{-1}(d c)+s^{-1} \bar{c}+(-1)^{c_{i}} s^{-1} c_{i} s^{-1} c^{i}\right) \\
& =\widetilde{\theta}\left(-s^{-1}(\widetilde{d} c)+(-1)^{c_{i}} s^{-1} c_{i} s^{-1} c^{i}\right) \\
& =\widetilde{\theta} \widetilde{d}_{\Omega}\left(s^{-1} c\right) .
\end{aligned}
$$

Algebraic 83 Geometric Topology, Volume 7 (2007) 
Furthermore,

$$
\begin{aligned}
D_{\Omega} \widetilde{\theta}\left(s^{-1} \bar{c}\right)= & D_{\Omega} \mathfrak{h} \theta\left(s^{-1} c\right) \\
= & -\mathfrak{h} d \theta\left(s^{-1} c\right) \\
= & -\mathfrak{h} \theta d_{\Omega}\left(s^{-1} c\right) \\
= & -\mathfrak{h} \theta\left(-s^{-1}(d c)+(-1)^{c_{i}} s^{-1} c_{i} s^{-1} c^{i}\right) \\
= & \widetilde{\theta}\left(s^{-1} \overline{d c}\right)-(-1)^{c_{i}} \mathfrak{h}\left(\theta\left(s^{-1} c_{i}\right) \theta\left(s^{-1} c^{i}\right)\right) \\
= & \widetilde{\theta}\left(-s^{-1} \widetilde{d} \bar{c}\right) \\
& -(-1)^{c_{i}}\left(\mathfrak{h} \theta\left(s^{-1} c_{i}\right) \cdot \mathfrak{s} \theta\left(s^{-1} c^{i}\right)+(-1)^{c_{i}+1} \mathfrak{s} \theta\left(s^{-1} c_{i}\right) \cdot \mathfrak{h} \theta\left(s^{-1} c^{i}\right)\right) \\
= & \widetilde{\theta}\left(-s^{-1} \widetilde{d} \bar{c}\right) \\
& -(-1)^{c_{i}}\left(\widetilde{\theta}\left(s^{-1} \overline{c_{i}}\right) \cdot \widetilde{\theta}\left(s^{-1} c^{i}\right)-(-1)^{c_{i}} \widetilde{\theta}\left(s^{-1} c_{i}\right) \cdot \widetilde{\theta}\left(s^{-1} \bar{c}^{i}\right)\right) \\
= & \widetilde{\theta}\left(-s^{-1} \widetilde{d} \bar{c}+(-1)^{c_{i}+1} s^{-1} \bar{c}_{i} s^{-1} c^{i}+(-1)^{c} s^{-1} c_{i} s^{-1} \bar{c}^{i}\right) \\
= & \widetilde{\theta} \widetilde{d}_{\Omega}\left(s^{-1} \bar{c}\right) .
\end{aligned}
$$

Observe that since $\widetilde{\theta}$ is a differential map, it is necessarily a quasi-isomorphism, as both $\mathfrak{P} \mathfrak{L}(C)$ and $\Omega H \otimes_{t_{\Omega}} H$ are acyclic.

(2) Let $\Theta \in(\mathcal{A}, \mathcal{F})-\operatorname{PsHopf}(\widetilde{\Omega}(C, \Psi), H)$ denote the pseudo $\mathcal{A}$-Hopf algebra map that $\theta$ underlies. Let $\mathfrak{F}(\Theta)=\left\{\theta_{k}: \Omega C \rightarrow H^{\otimes k} \mid k \geq 1\right\}$, where $\theta_{k}=\Theta\left(-\otimes z_{k-1}\right)$. For $k \geq 0$, define

$$
\widetilde{\theta}_{k}: s^{-1}(C \oplus \bar{C}) \rightarrow\left(\Omega H \otimes_{t_{\Omega}} H\right) \otimes H^{\otimes k}
$$

by

$$
\widetilde{\theta}_{k}\left(s^{-1} c\right)=\left(\mathfrak{s} \otimes 1^{\otimes k}\right) \theta_{k+1}\left(s^{-1} c\right)
$$

and

$$
\widetilde{\theta}_{k}\left(s^{-1} \bar{c}\right)=\left(\mathfrak{h} \otimes 1^{\otimes k}\right) \theta_{k+1}\left(s^{-1} c\right),
$$

where 1 denotes the identity on $H$.

We claim that

$$
\left\{\widetilde{\theta}_{k}: s^{-1}(C \oplus \bar{C}) \rightarrow\left(\Omega H \otimes_{t_{\Omega}} H\right) \otimes H^{\otimes k} \mid k \geq 1\right\}
$$

satisfies the hypotheses of Proposition 3.16 and therefore induces a morphism

$$
\widetilde{\Theta} \in(\mathcal{A}, \mathcal{F})-\operatorname{PsHopf}\left(\mathfrak{P} \mathfrak{L}(C, \Psi), H \otimes_{t_{\Omega}} \Omega H\right),
$$

ie, $\widetilde{\theta}$ is a CASH map. We prove this claim by induction on $k$ and on degree in the Appendix.

Algebraic 8 Geometric Topology, Volume 7 (2007) 
Note that $\widetilde{\Theta}$ lifts $\Theta$, in the sense that $\mathfrak{p}^{\otimes n} \widetilde{\Theta}(n)=\Theta(n)\left(\widetilde{\Omega} \pi \diamond 1_{\mathcal{F}}\right)$ for all $n$. This is a necessary condition for $\widetilde{\theta}$ to be a CASH map with respect to $\theta$.

Summary 5.5 Given an object $H$ of $\mathbf{H}$ and an Alexander-Whitney model of $H$

$$
\theta: \widetilde{\Omega}(C, \Psi) \stackrel{\simeq}{\longrightarrow} H,
$$

there exists a commutative diagram

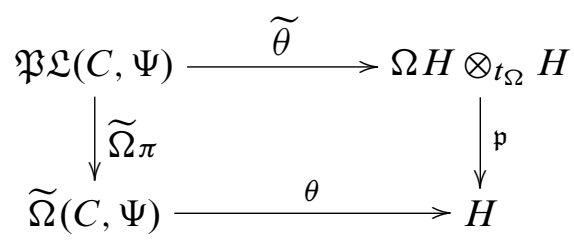

such that

(1) $\widetilde{\Omega} \pi$ is a strict algebra and coalgebra map;

(2) $\mathfrak{p}$ is a strict algebra and right $H$-comodule map;

(3) the natural right $\Omega C$-comodule structure on $\mathfrak{P} \mathfrak{L}(C, \Psi)$ is cofree on $\mathfrak{P} \mathfrak{L}(C, \Psi) \square_{\Omega C} R$; and

(4) $\widetilde{\theta}$ is a quasi-isomorphism that is a strict algebra map and a CASH map.

Furthermore, this construction is natural in $\theta$.

The next theorem, which is the heart of this article, describes how we can use the pathloop construction to compute the multiplicative structure of homotopy fiber homologies in $\mathbf{H}$. Recall from Corollary 3.8 that if $H$ is a chain Hopf algebra and $M$ is an $H$-comodule algebra, then $\operatorname{Cotor}^{H}(M, R)=\mathrm{H}_{*}\left(M \otimes_{t_{\Omega}} \Omega H\right)$ has a natural graded algebra structure. Recall furthermore from Corollary 4.7 that

$$
\widetilde{\Omega}\left(\left(C^{\prime}, \Psi^{\prime}\right) \bigsqcup \mathfrak{P}(C, \Psi)\right) \square \widetilde{\Omega}(C, \Psi) R
$$

is a sub chain algebra of $\widetilde{\Omega}\left(\left(C^{\prime}, \Psi^{\prime}\right) \bigsqcup \mathfrak{P}(C, \Psi)\right)$ for all $\theta \in \mathbf{F}\left(\left(C^{\prime}, \Psi^{\prime}\right),(C, \Psi)\right)$.

Theorem 5.6 Let $\varphi: H^{\prime} \rightarrow H$ be a map of chain Hopf algebras. Suppose that there is a a map $\omega:\left(C^{\prime}, \Psi^{\prime}\right) \rightarrow(C, \Psi)$ in $\mathbf{F}$ and a commutative diagram

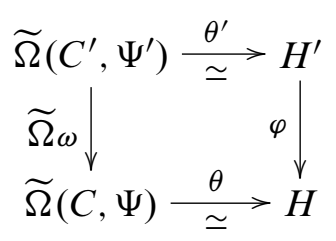

Algebraic $8 \mathcal{G}$ Geometric Topology, Volume 7 (2007) 
in which $\theta^{\prime}$ and $\theta$ are Alexander-Whitney models. Let

$$
\mathfrak{L} \mathfrak{F}(\omega)=\widetilde{\Omega}\left(C^{\prime} \coprod \mathfrak{P}(C)\right) \square_{\Omega C} R .
$$

Then there is a zig-zag of quasi-isomorphisms of chain algebras

$$
\mathfrak{L F}(\omega) \stackrel{\simeq}{\simeq} \stackrel{\simeq}{\simeq} \cdot \stackrel{\simeq}{\leftrightarrows} H^{\prime} \otimes_{t_{\Omega}} \Omega H .
$$

In particular, $\mathrm{H}_{*}(\mathfrak{L} \mathfrak{F}(\omega))$ is isomorphic to $\operatorname{Cotor}^{H}\left(H^{\prime}, R\right)$ as graded algebras.

It is not surprising that there is at least a linear isomorphism between $\mathrm{H}_{*}(\mathfrak{L F}(\omega))$ and $\operatorname{Cotor}^{H}\left(H^{\prime}, R\right)$. Since $\widetilde{\Omega}\left(\left(C^{\prime}, \Psi^{\prime}\right) \bigsqcup \mathfrak{P}(C, \Psi)\right)$ is a $\widetilde{\Omega}(C, \Psi)$-cofree resolution of $\widetilde{\Omega}\left(C^{\prime}, \Psi^{\prime}\right)$ and Cotor is the derived functor of the cotensor product, $\operatorname{Cotor} \widetilde{\Omega}(C, \Psi)\left(\widetilde{\Omega}\left(C^{\prime}, \Psi^{\prime}\right), R\right)$ should be the same as $\mathrm{H}_{*}(\mathfrak{L} \mathfrak{F}(\omega))$. Naturality then gives rise to the linear isomorphism $\mathrm{H}_{*}(\mathfrak{L F}(\omega))$ and $\operatorname{Cotor}^{H}\left(H^{\prime}, R\right)$. The challenge lies in showing that the isomorphism is multiplicative.

Proof The factorization of $\omega$ described in (4-5)

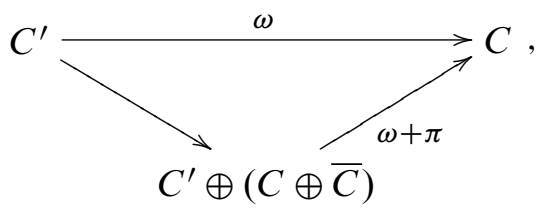

induces a factorization of $\widetilde{\Omega} \omega$

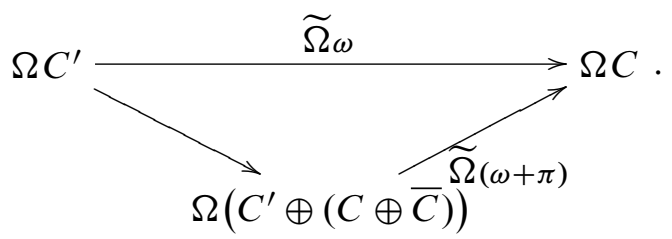

Note that $\widetilde{\Omega}(\omega+\pi)$ admits a (nondifferential) Hopf algebra section $\Omega \mathbf{j}$, where $\mathbf{j}$ is the natural section of $\omega+\pi$. We can therefore apply Proposition 4.6 to $\widetilde{\Omega}(\omega+\pi)$.

From Proposition 4.6, we know that there is an injection of chain algebras

$$
i: \mathfrak{L F}(\omega) \hookrightarrow \Omega\left(C^{\prime} \oplus(C \oplus \bar{C})\right)
$$

and an isomorphism of left $\mathfrak{L} \mathfrak{F}(\omega)$-modules and right $\Omega C$-comodules

$$
h: \mathfrak{L} \mathfrak{F}(\omega) \otimes \Omega C \rightarrow \Omega\left(C^{\prime} \oplus(C \oplus \bar{C})\right)
$$

defined by $h=\mu(i \otimes \Omega \mathfrak{j})$. 
Next, using the right $\Omega C$-comodule structure of $\Omega\left(C^{\prime} \oplus(C \oplus \bar{C})\right)$, define the twisted tensor product $\Omega\left(C^{\prime} \oplus(C \oplus \bar{C})\right) \otimes_{t_{\Omega}} \Omega^{2} C$, and let

$$
\eta: \Omega\left(C^{\prime} \oplus(C \oplus \bar{C})\right) \hookrightarrow \Omega\left(C^{\prime} \oplus(C \oplus \bar{C})\right) \otimes_{t_{\Omega}} \Omega^{2} C
$$

denote the natural (nondifferential) injection of algebras such that $\eta(w)=w \otimes 1$. We claim that the composition

$$
\eta i: \mathfrak{L F}(\omega) \rightarrow \Omega\left(C^{\prime} \oplus(C \oplus \bar{C})\right) \otimes_{t_{\Omega}} \Omega^{2} C
$$

is a quasi-isomorphism of chain algebras.

Note that $\eta i$ factors as a composite of chain maps

$$
\mathfrak{L F}(\omega) \stackrel{\text { incl. }}{\longrightarrow}(\mathfrak{L F}(\omega) \otimes \Omega C) \otimes_{t_{\Omega}} \Omega^{2} C \stackrel{h \otimes 1}{\longrightarrow} \Omega\left(C^{\prime} \oplus(C \oplus \bar{C})\right) \otimes_{t_{\Omega}} \Omega^{2} C .
$$

The linear map $h \otimes 1$ is a differential map because $h$ is a map of differential $\Omega C$ comodules, while the inclusion $\mathfrak{L} \mathfrak{F}(\omega) \hookrightarrow(\mathfrak{L F}(\omega) \otimes \Omega C) \otimes_{t_{\Omega}} \Omega^{2} C$ is a differential map since $\mathfrak{L F}(\omega) \otimes \Omega C$ is cofree. Since $\Omega C \otimes_{t_{\Omega}} \Omega^{2} C$ is acyclic, the first, inclusion map is a quasi-isomorphism. The second map is also a quasi-isomorphism, as $h$ is an isomorphism. Thus, $\eta i$ is a quasi-isomorphism, as claimed.

Similarly, since there is a chain subalgebra inclusion

$$
H^{\prime} \otimes_{t_{\Omega}} \Omega H \hookrightarrow H^{\prime} \otimes_{t_{\Omega}}\left(\Omega H \otimes_{t_{\Omega}} H\right) \otimes_{t_{\Omega}} \Omega H
$$

which is a quasi-isomorphism since $H \otimes_{t_{\Omega}} \Omega H$ is acyclic.

The conditions on diagrams (5-1) and (5-2) imply that we have morphisms in CASH (cf, Definition 3.14)

$$
(\theta ; \widetilde{\theta}):(\widetilde{\Omega}(C, \Psi) ; \mathfrak{P} \mathfrak{L}(C, \Psi)) \rightarrow\left(H ; \Omega H \otimes_{t_{\Omega}} H\right)
$$

and

$$
\left(\theta ; \theta^{\prime}\right):\left(\widetilde{\Omega}(C, \Psi) ; \widetilde{\Omega}\left(C^{\prime}, \Psi^{\prime}\right)\right) \rightarrow\left(H ; H^{\prime}\right) .
$$

Applying Proposition 3.18, we obtain a CASH map

$$
\theta^{\prime} \star \widetilde{\theta}: \Omega\left(C^{\prime} \oplus(C \oplus \bar{C})\right) \rightarrow H^{\prime} \otimes_{t_{\Omega}}\left(\Omega H \otimes_{t_{\Omega}} H\right),
$$

associated to a chain algebra map

$$
\zeta: \Omega\left(C^{\prime} \oplus(C \oplus \bar{C})\right) \otimes_{t_{\Omega}} \Omega^{2} C \longrightarrow H^{\prime} \otimes_{t_{\Omega}}\left(\Omega H \otimes_{t_{\Omega}} H\right) \otimes_{t_{\Omega}} \Omega H .
$$


Since $\Omega(C \oplus \bar{C})$ and $\Omega H \otimes_{t_{\Omega}} H$ are acyclic, and $\Omega\left(C^{\prime} \oplus(C \oplus \bar{C})\right) \cong \Omega C^{\prime} \amalg \Omega(C \oplus$ $\bar{C})$, the vertical arrows in the commuting diagram

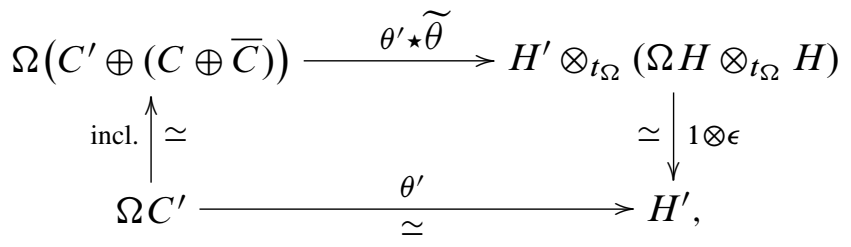

where $\epsilon$ denotes the augmentation map, are both quasi-isomorphisms. Consequently, $\theta^{\prime} \star \widetilde{\theta}$ is a quasi-isomorphism, which implies that $\zeta$ is also a quasi-isomorphism, since the underlying graded modules of all objects involved are assumed to be free over $R$.

We have therefore a zig-zag of quasi-isomorphisms of chain algebras

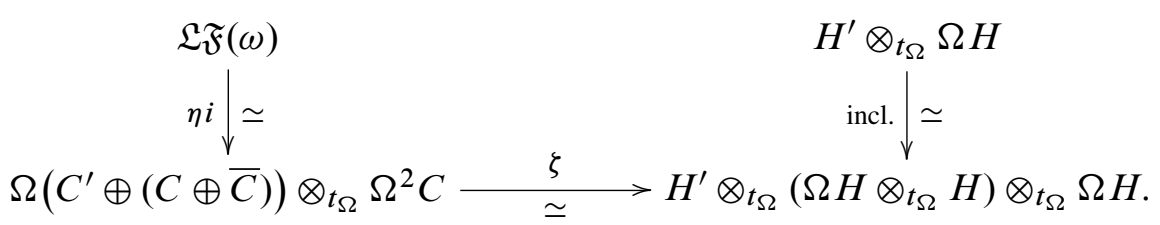

Consequently,

$$
\mathrm{H}_{*} \mathfrak{L F}(\omega) \cong \mathrm{H}_{*}\left(H^{\prime} \otimes_{t_{\Omega}} \Omega H\right)=\operatorname{Cotor}^{H}\left(H^{\prime}, R\right)
$$

as graded algebras.

Corollary 5.7 Let $\theta: \widetilde{\Omega}(C, \Psi) \rightarrow H$ be an Alexander-Whitney model of a chain Hopf algebra $H$. If

$$
\mathfrak{L}_{2}(C, \Psi)=\mathfrak{P} \mathfrak{L}(C, \Psi) \square \widetilde{\Omega}_{(C, \Psi)} R,
$$

with its natural chain algebra structure inherited from $\widetilde{\Omega}(C, \Psi)$, then $\mathrm{H}_{*}\left(\mathfrak{L}_{2}(C, \Psi)\right)$ is isomorphic to $\operatorname{Cotor}^{H}(R, R)$ as graded algebras.

Proof Apply Theorem 5.6 to the unit map $R \rightarrow H$.

Definition 5.8 Given an object $(C, \Psi)$ in $\mathbf{F}$, the chain algebra $\mathfrak{L}_{2}(C, \Psi)$ is the doubleloop construction on $C$. Given a morphism $\omega:\left(C^{\prime}, \Psi^{\prime}\right) \rightarrow(C, \Psi)$ of $\mathbf{F}$, the chain algebra $\mathfrak{L} \mathfrak{F}(\omega)$ is the loop-homotopy fiber construction on $\omega$.

Remark 5.9 Note that $\mathfrak{L}_{2}(C, \Psi)$ is a subalgebra of $\mathfrak{P} \mathfrak{L}(C, \Psi)=\widetilde{\Omega} \circ \widetilde{\mathfrak{P}}(C, \Psi)$, which is free as a graded algebra on $C \oplus \bar{C}$. In particular, if $C$ admits an $R$-basis of $n$ elements, then $\mathfrak{L}_{2}(C, \Psi)$ is a subalgebra of a free algebra on $2 n$ generators. On the 
other hand, $\Omega(\widetilde{\Omega}(C, \Psi))$, which is connected by a zig-zag of quasi-isomorphisms of chain algebras to $\mathfrak{L}_{2}(C, \Psi)$, is free on an infinite number of generators.

A similar comparison can be made between $\mathfrak{L F}(\omega)$ and $\Omega\left(C^{\prime} \oplus(C \oplus \bar{C})\right) \otimes_{t_{\Omega}} \Omega^{2} C$.

Remark 5.10 Since the path-loop construction is functorial, the double-loop construction is as well, ie, a morphism $h:(C, \Psi) \rightarrow\left(C^{\prime}, \Psi^{\prime}\right)$ in $\mathbf{F}$ induces a chain algebra map $\mathfrak{L}_{2}(h): \mathfrak{L}_{2}(C, \Psi) \rightarrow \mathfrak{L}_{2}\left(C^{\prime}, \Psi^{\prime}\right)$. It is evident that if $h$ is a quasi-isomorphism in $\mathbf{F}$, then $\mathfrak{L}_{2}(h)$ is a quasi-isomorphism of chain algebras.

Similarly, the loop-homotopy fiber construction is clearly defines a functor from the category of morphisms in $\mathbf{F}$ to the category of chain algebras. Furthermore if

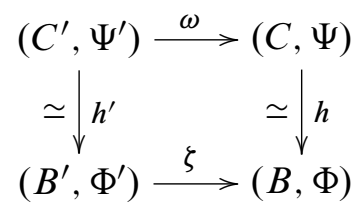

is a commuting diagram in $\mathbf{F}$, where $h$ and $h^{\prime}$ are quasi-isomorphisms, then the induced map $\mathfrak{L} \mathfrak{F}(\omega) \rightarrow \mathfrak{L} \mathfrak{F}(\zeta)$ is a quasi-isomorphism of chain algebras.

\section{The loops on a homotopy fiber}

We are now ready to apply the purely algebraic results above to topology.

\subsection{The loop-homotopy fiber model}

Here we apply the constructions and theorems of the previous two sections to constructing a chain algebra, the homology of which is isomorphic as a graded algebra to $\mathrm{H}_{*}(G F)$, where $F$ is the homotopy fiber of a morphism $g: K \rightarrow L$ of 2 -reduced simplicial sets.

We begin by specifying our input data for the constructions of Section 4 and Section 5: the canonical enriched Adams-Hilton model of [8].

Recall from Theorem 2.13 that there is a functor $\widetilde{C}:$ sSet $_{1} \rightarrow$ F. In [16], Szczarba gave an explicit formula for a natural transformation between functors from $\mathbf{s S e t}_{1}$ to the category of associative chain algebras

$$
\theta: \Omega C(-) \rightarrow C(G(-))
$$

such that $\theta_{K}: \Omega C(K) \rightarrow C(G K)$ is a quasi-isomorphism of chain algebras for every 1 -reduced simplicial set $K$. 
Since $\Omega$ extends to a functor $\widetilde{\Omega}: \mathbf{F} \rightarrow \mathbf{H}$ (see (1.5)), there is a natural transformation $\psi: \Omega C(-) \rightarrow \Omega C(-) \otimes \Omega C(-)$ given for each 1 -reduced simplicial set $K$ by the composition

$$
\Omega C(K) \stackrel{\operatorname{Ind}\left(\Psi_{K}\right)}{\longrightarrow} \Omega(C(K) \otimes C(K)) \stackrel{q}{\rightarrow} \Omega C(K) \otimes \Omega C(K),
$$

where $\widetilde{C}(K)=\left(C(K), \Psi_{K}\right)$. The comultiplication $\psi_{K}: \Omega C(K) \rightarrow \Omega C(K) \otimes \Omega C(K)$ is called the Alexander-Whitney cobar diagonal.

In [8] Hess, Parent, Scott and Tonks established that Szczarba's equivalence $\theta_{\boldsymbol{K}}$ underlies a pseudo $\mathcal{A}$-Hopf map with respect to $\psi_{K}$ and the usual comultiplication on $C(G K)$. In other words, for all 1 -reduced $K$,

$$
\theta_{K}: \widetilde{\Omega} \widetilde{C}(K) \stackrel{\simeq}{\rightarrow} C(G K)
$$

is an Alexander-Whitney model, called the canonical enriched Adams-Hilton model of $K$. Finally, they showed that $\psi_{K}$ agrees with the comultiplication on $\Omega C(K)$ defined in a purely combinatorial manner by Baues in [1].

We now apply the canonical enriched Adams-Hilton model to modelling the loop homology of homotopy fibers.

Theorem 6.1 Let $f: K \rightarrow L$ be a morphism of 2 -reduced simplicial sets, and let $F$ be the homotopy fiber of $f$. Then there is a zig-zag of quasi-isomorphisms of chain algebras

$$
\mathfrak{L F}(f) \stackrel{\simeq}{\longleftarrow} \stackrel{\simeq}{\rightarrow} \cdots \stackrel{\simeq}{\simeq} \stackrel{\simeq}{\rightarrow} C(G F) .
$$

Thus,

$$
\mathrm{H}_{*}(\mathfrak{L F}(\widetilde{C}(f))) \cong \mathrm{H}_{*}(G F)
$$

as graded algebras. In particular,

$$
\mathrm{H}_{*}\left(\mathfrak{L}_{2}(\widetilde{C}(L))\right) \cong \mathrm{H}_{*}\left(G^{2} L\right)
$$

as graded algebras.

Proof Applying Theorem 5.6 to the commuting diagram

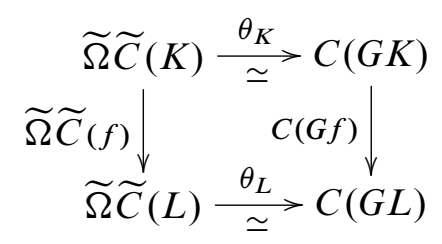

Algebraic 83 Geometric Topology, Volume 7 (2007) 
we obtain a zig-zag of quasi-isomorphisms of chain algebras

$$
\mathfrak{L F}(f) \stackrel{\simeq}{\longleftarrow} \stackrel{\simeq}{\rightarrow} \cdots \stackrel{\simeq}{\longleftarrow} \stackrel{\simeq}{\rightarrow} C(G K) \otimes_{t_{\Omega}} \Omega C(G L) .
$$

By the dual of [4, Theorem 5.1], there is a quasi-isomorphism of chain algebras

$$
C(G F) \stackrel{\simeq}{\rightarrow} C(G K) \otimes_{t_{\Omega}} \Omega C(G L),
$$

and so we can conclude.

\subsection{Double suspensions and formal spaces}

In this section we provide a more explicit description of $\mathfrak{L}_{2}(C, \Psi)$ for AlexanderWhitney coalgebras $(C, \Psi)$ such that $\widetilde{\Omega}(C, \Psi)$ is primitively generated. Since $\widetilde{\Omega} \widetilde{C}(K)$ is primitively generated for all simplicial double suspensions $K$ [7], we have good control of the model $\mathfrak{L}_{2}(K)$ for a large class of spaces $K$.

Our description of $\mathfrak{L}_{2}(C, \Psi)$ also applies to Alexander-Whitney coalgebras that are "formal" in some appropriate sense. The chain coalgebras of numerous interesting spaces satisfy our formality criteria, enabling us to give a more explicit and computationally amenable formula for the model of their double loop spaces as well.

Our notion of formality is certainly closely related to that of Ndombol and Thomas [14], but we do not know whether the two notions are actually equivalent.

Definition 6.2 A morphism $\theta \in \mathbf{F}\left((C, \Psi),\left(C^{\prime}, \Psi^{\prime}\right)\right)$ is an $\mathbf{F}$-quasi-isomorphism if $\theta\left(-\otimes z_{0}\right): C \rightarrow C^{\prime}$ is a quasi-isomorphism. Two Alexander-Whitney coalgebras $(C, \Psi)$ and $\left(C^{\prime}, \Psi^{\prime}\right)$ are weakly equivalent if there is a zig-zag of $\mathbf{F}$-quasiisomorphisms in $\mathbf{F}$

$$
(C, \Psi)<\simeq \stackrel{\simeq}{\longrightarrow} \cdots<\simeq \stackrel{\simeq}{\longrightarrow}\left(C^{\prime}, \Psi^{\prime}\right) .
$$

Let $(C, \Psi)$ and $\left(C^{\prime}, \Psi^{\prime}\right)$ be weakly equivalent Alexander-Whitney coalgebras, with underlying coalgebras $(C, \Delta)$ and $\left(C^{\prime}, \Delta^{\prime}\right)$. It follows immediately from the definition that $H_{*}(C, \Delta) \cong H_{*}\left(C^{\prime}, \Delta^{\prime}\right)$ as cocommutative coalgebras and that $H_{*}(\widetilde{\Omega}(C, \Psi)) \cong$ $H_{*}\left(\widetilde{\Omega}\left(C^{\prime}, \Psi^{\prime}\right)\right)$ as Hopf algebras.

Recall the "inclusion" functor $\mathfrak{I}_{\mathcal{F}}: \mathcal{A}$-Coalg $\rightarrow(\mathcal{A}, \mathcal{F})$-Coalg from $(1-1)$.

Definition 6.3 An Alexander-Whitney coalgebra $(C, \Psi)$ is formal if it is weakly equivalent to $\mathfrak{I}_{\mathcal{F}}\left(\mathrm{H}_{*}(C)\right)$. A simplicial set $K$ is Alexander-Whitney formal if $\widetilde{C}(K)$ formal in $\mathbf{F}$. 
Spheres are obviously Alexander-Whitney formal spaces. More generally, if $K$ is an $r$-reduced simplicial set such that all simplices of dimension greater than $2 r$ are degenerate, then $K$ is clearly Alexander-Whitney formal for degree reasons.

We now recall algebraic notions and results from [2] and [17] that enable us to simplify $\mathfrak{L}_{2}(C, \Psi)$ in the formal case. We work henceforth over any integral domain $R$ in which 2 is a unit or over a field $R$ of characteristic 2 .

Definition 6.4 (Cohen, Moore and Neisendorfer [2, Section 3]) A homology Hopf algebra is a connected, cocommutative, graded Hopf algebra that is free as a graded $R-$ module. Given homology Hopf algebras $H, H^{\prime}$ and $H^{\prime \prime}$, a sequence $H^{\prime} \stackrel{i}{\rightarrow} H \stackrel{p}{\rightarrow} H^{\prime \prime}$ of morphisms of Hopf algebras is a short exact sequence if

(1) the composite $p i$ is equal to the composite $H^{\prime} \stackrel{\varepsilon}{\rightarrow} R \stackrel{\eta}{\rightarrow} H^{\prime \prime}$;

(2) $i$ is injective, while $p$ is surjective; and

(3) the canonical map $\bar{l}: H^{\prime} \rightarrow H \square_{H^{\prime \prime}} R$ is an isomorphism.

Proposition 6.5 (Cohen, Moore and Neisendorfer [2, Proposition 3.7]) If $L^{\prime} \rightarrow$ $L \rightarrow L^{\prime \prime}$ is a short exact sequence of connected, graded Lie algebras over $R$, then $U L^{\prime} \rightarrow U L \rightarrow U L^{\prime \prime}$ is a short exact sequence of homology Hopf algebras, where $U$ denotes the universal enveloping algebra functor, from graded Lie algebras to graded Hopf algebras.

Proposition 6.6 (Tanré [17, Proposition VI.2 (7)]) Let $p: \mathbb{L}(V \oplus W) \rightarrow \mathbb{L}(V)$ be the projection map of graded Lie algebras, determined by $p(v)=v$ and $p(w)=0$ for all $v \in V$ and $w \in W$. There is then a short exact sequence of Lie algebras

$$
\mathbb{L}\left(\mathfrak{A}^{\mathbb{N}}(V)(W)\right) \stackrel{i}{\rightarrow} \mathbb{L}(V \oplus W) \stackrel{p}{\rightarrow} \mathbb{L}(V),
$$

where

$$
\mathfrak{A}^{\mathbb{N}}(V)(W):=\left\{\left[v_{1},\left[v_{2},\left[\ldots\left[v_{k}, w\right] \ldots\right]\right]\right] \mid w \in W, v_{i} \in V \forall i, k \in \mathbb{N}\right\}
$$

and $[-,-]$ denotes a commutator.

We can now apply the results recalled above to determining the underlying graded algebra of $\mathfrak{L}_{2}(C, \Psi)$ when $\widetilde{\Omega}(C, \Psi)$ is primitively generated.

Theorem 6.7 Let $R$ be either an integral domain in which 2 is a unit or a field of characteristic 2. If $(C, \Psi)$ is an Alexander-Whitney coalgebra over $R$ such that $\widetilde{\Omega}(C, \Psi)$ is primitively generated with respect to the naturally induced comultiplication $\psi$, then the underlying graded algebra of $\mathfrak{L}_{2}(C, \Psi)$ is $T\left(\mathfrak{A}^{\mathbb{N}}\left(s^{-1} C\right)\left(s^{-1} \bar{C}\right)\right)$. 
Proof Since the underlying graded Hopf algebra of $\widetilde{\Omega}(C, \Psi)$ is primitively generated and therefore cocommutative, so is the underlying graded Hopf algebra of $\mathfrak{P L}(C, \Psi)$. It follows that there is a short exact sequence of homology Hopf algebras

$$
T s^{-1}\left(C_{+} \oplus \bar{C}_{+}\right) \square_{T s^{-1} C_{+}} R \rightarrow T s^{-1}\left(C_{+} \oplus \bar{C}_{+}\right) \stackrel{\Omega \pi}{\longrightarrow} T s^{-1} C_{+},
$$

where $T s^{-1}\left(C_{+} \oplus \bar{C}_{+}\right) \square_{T s^{-1} C_{+}} R$ is the graded Hopf algebra underlying $\mathfrak{L}_{2}(C, \Psi)$.

On the other hand, since each of the Hopf algebras above is primitively generated, $\Omega \pi=U p$, where $p: \mathbb{L} s^{-1}\left(C_{+} \oplus \bar{C}_{+}\right) \rightarrow \mathbb{L} s^{-1} C_{+}$is the usual projection map of graded Lie algebras. Combining Proposition 6.5 and Proposition 6.6, we obtain another short exact sequence of homology Hopf algebras

$$
T\left(\mathfrak{A}^{\mathbb{N}}\left(s^{-1} C\right)\left(s^{-1} \bar{C}\right)\right) \rightarrow T s^{-1}\left(C_{+} \oplus \bar{C}_{+}\right) \stackrel{\Omega \pi}{\longrightarrow} T s^{-1} C_{+} .
$$

Comparing with sequence (6-1), we conclude that

$$
T s^{-1}\left(C_{+} \oplus \bar{C}_{+}\right) \square_{T s^{-1} C_{+}} R \cong T\left(\mathfrak{A}^{\mathbb{N}}\left(s^{-1} C\right)\left(s^{-1} \bar{C}\right)\right) .
$$

Corollary 6.8 Let $R$ be either an integral domain in which 2 is a unit or a field of characteristic 2. If $K=E^{2} L$ is a simplicial double suspension, then the graded algebra underlying $\mathfrak{L}_{2}(K)$ is $T\left(\mathfrak{A}^{\mathbb{N}}\left(C_{+} E L\right)\left(C_{+} L\right)\right)$.

Proof In [7] the authors proved that $\widetilde{\Omega} \widetilde{C}\left(E^{2} L\right)$ was primitively generated for all simplicial sets $L$. To conclude, observe that $s^{-1} C_{+} E X \cong C_{+} X$ for all simplicial sets $X$.

Corollary 6.9 Let $R$ be either an integral domain in which 2 is a unit or is weakly equivalent to a chain algebra with underlying graded algebra

Proof Writing $\Im(H)=\left(H, \Psi_{H}\right)$, we have by definition that $\Psi_{H}=\mathcal{T}\left(\Delta_{H}\right) \diamond_{\mathcal{A}} \varepsilon$. The induced comultiplication on $\widetilde{\Omega}\left(H, \Psi_{H}\right)$ is such that $\widetilde{\Omega}\left(H, \Psi_{H}\right)$ is primitively generated. Now apply Theorem 6.7 .

If $(C, \Psi)$ is formal and all elements of $\mathrm{H}_{*}(C)$ are primitive, then the differential $\widetilde{d}_{\Omega}$ on $\mathfrak{L}_{2}\left(H, \Psi_{H}\right)$ is given explicitly by

$$
\begin{aligned}
& \widetilde{d}_{\Omega}\left(\left[s^{-1} x_{1},\left[s^{-1} x_{2},\left[\ldots\left[s^{-1} x_{m}, s^{-1} \bar{y}\right] \ldots\right]\right]\right]\right) \\
&=\sum_{1 \leq i \leq m} \pm\left[s^{-1} x_{1},\left[s^{-1} x_{2},\left[\ldots\left[s^{-1} \bar{x}_{i},\left[\ldots\left[s^{-1} x_{m}, s^{-1} \bar{y}\right] \ldots\right]\right]\right],\right.\right.
\end{aligned}
$$


for all $x_{1}, \ldots, x_{m}, y \in H$, where the sign is determined by the Koszul convention. It is not too difficult to see in this case that if $B$ is a basis of $\mathrm{H}_{*}\left(C, \mathbb{F}_{2}\right)$, then

$$
\mathrm{H}_{*}\left(\mathfrak{L}_{2}(C, \Psi) ; \mathbb{F}_{2}\right) \cong \mathbb{F}_{2}\left[\operatorname{ad}^{2^{k}-1}(x)(\bar{x}) \mid x \in B, k \geq 1\right] .
$$

A similar result holds mod $p$.

\section{Appendix A Technical proofs}

\section{A.1 Proof of Proposition 2.5}

Since $\mathcal{Q}$ is free as a $\mathcal{P}$-bimodule, there is a symmetric sequence $\mathcal{X}$ of graded $R-$ modules such that $\mathcal{Q} \cong \mathcal{P} \diamond \mathcal{X} \diamond \mathcal{P}$ in ${ }_{\mathcal{P}} \mathbf{M o d}_{\mathcal{P}}$.

The morphism $\theta$ consists of a morphism of right $\mathcal{P}$-modules

$$
\theta: \mathcal{T}(H) \diamond_{\mathcal{P}} \mathcal{Q} \rightarrow \mathcal{T}\left(H^{\prime}\right)
$$

such that

$$
\theta \mathfrak{I}_{\mathcal{Q}}(\mu)=\mathfrak{I}_{\mathcal{Q}}\left(\mu^{\prime}\right)(\theta \curlywedge \theta): \mathcal{T}(H \wedge H) \diamond_{\mathcal{P}} \mathcal{Q} \rightarrow \mathcal{T}\left(H^{\prime}\right),
$$

where the $\mathcal{P}$-coalgebra maps $\mu$ and $\mu^{\prime}$ are the multiplication maps on $H$ and $H^{\prime}$, respectively.

Let $\rho: \mathcal{T}(H \coprod T v) \diamond \mathcal{P} \rightarrow \mathcal{T}(H \coprod T v)$ be the right $\mathcal{P}$-module structure corresponding to the $\mathcal{P}$-coalgebra structure of $H \bigsqcup T v$. Since $H \bigsqcup T v$ is an extension of $H$, the action $\rho$ restricts to $\rho: \mathcal{L}(R \cdot v) \diamond \mathcal{P} \rightarrow \mathcal{T}(H \oplus R \cdot v)$. In particular, the direct sum of graded modules $H \oplus R \cdot v$ underlies a sub $\mathcal{P}$-coalgebra of $H \amalg T v$.

Taken together, $\lambda: \mathcal{L}(R \cdot v) \diamond \mathcal{X} \rightarrow \mathcal{T}\left(H^{\prime}\right)$ and the restriction $\theta: \mathcal{L}(H) \diamond \mathcal{X} \rightarrow \mathcal{T}\left(H^{\prime}\right)$ give rise in the obvious way to a morphism of symmetric sequences from $\mathcal{L}(H \oplus R$. $v) \diamond \mathcal{X}$ to $\mathcal{T}\left(H^{\prime}\right)$, which we call $\theta \boxplus \lambda$, and then, by Lemma 2.3, to

$$
(\theta \boxplus \lambda): \mathcal{T}(H \oplus R \cdot v) \diamond \mathcal{X} \rightarrow \mathcal{T}\left(H^{\prime}\right) .
$$

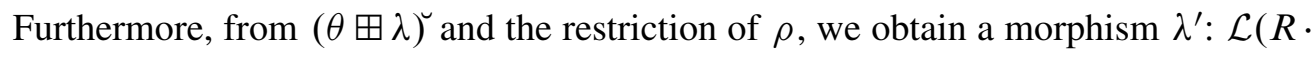
$v) \diamond \mathcal{Q} \rightarrow \mathcal{T}\left(H^{\prime}\right)$ of right $\mathcal{P}$-modules as the composite

$$
\mathcal{L}(R \cdot v) \diamond \mathcal{P} \diamond \mathcal{X} \diamond \mathcal{P} \stackrel{\rho \diamond 1 \diamond 1}{\longrightarrow} \mathcal{T}(H \oplus R \cdot v) \diamond \mathcal{X} \diamond \mathcal{P} \stackrel{(\theta \boxplus \lambda) \triangleleft 1}{\longrightarrow} \mathcal{T}\left(H^{\prime}\right) \diamond \mathcal{P} \stackrel{\rho^{\prime}}{\rightarrow} \mathcal{T}\left(H^{\prime}\right),
$$

where $\rho^{\prime}$ is the right $\mathcal{P}$-module structure map corresponding to the $\mathcal{P}$-coalgebra structure of $H^{\prime}$.

We next recursively define a filtration of $H \amalg T v$ by sub $\mathcal{P}$-coalgebras, then construct $\widehat{\theta}$ by induction on filtration degree. Set $F^{0}=H$ and, for $m>0, F^{m}$ is the image of 
the iterated multiplication map restricted to $F^{m-1} \otimes(R \oplus R \cdot v) \otimes H$. By definition of a free algebraic $\mathcal{P}$-Hopf extension, each $F^{m}$ is a sub $\mathcal{P}$-coalgebra of $H \bigsqcup T v$. Furthermore the multiplication $\mu$ on $H \amalg T v$ restricts to a morphism of $\mathcal{P}$-coalgebras

$$
\mu_{k, m}: F^{k} \otimes F^{m-k} \rightarrow F^{m},
$$

for all $0 \leq k \leq m$ and for all $m \geq 0$.

Let $\widehat{\theta}^{(0)}=\theta$. Suppose that for some $m \geq 0$ and for all $k \leq m$, there exists

$$
\widehat{\theta}^{(k)}: \mathcal{T}\left(F^{k}\right) \diamond_{\mathcal{P}} \mathcal{Q} \rightarrow \mathcal{T}\left(H^{\prime}\right)
$$

such that for all $j \leq k, \widehat{\theta}^{(k)}$ agrees with $\widehat{\theta}^{(j)}$ on $\mathcal{T}\left(F^{j}\right) \diamond_{\mathcal{P}} \mathcal{Q}$ and

$$
(\mathrm{A}-1) \quad \widehat{\theta}^{(k)} \Im\left(\mu_{j, k}\right)=\Im\left(\mu^{\prime}\right)\left(\widehat{\theta}^{(j)} \wedge \widehat{\theta}^{(k-j)}\right): \mathcal{T}\left(F^{j} \wedge F^{k-j}\right) \diamond_{\mathcal{P}} \mathcal{Q} \rightarrow \mathcal{T}\left(H^{\prime}\right) \text {. }
$$

To construct $\widehat{\theta}^{(m+1)}$, we begin by defining a morphism $\theta^{(m+1)}$ in $\operatorname{Mod}_{\boldsymbol{R}}^{\Sigma}$ from $\mathcal{L}\left(F^{m+1}\right) \diamond \mathcal{X}$ to $\mathcal{T}\left(H^{\prime}\right)$, to which we then apply Lemma 2.3. The morphism $\theta^{(m+1)}$ is defined to be the following composite.

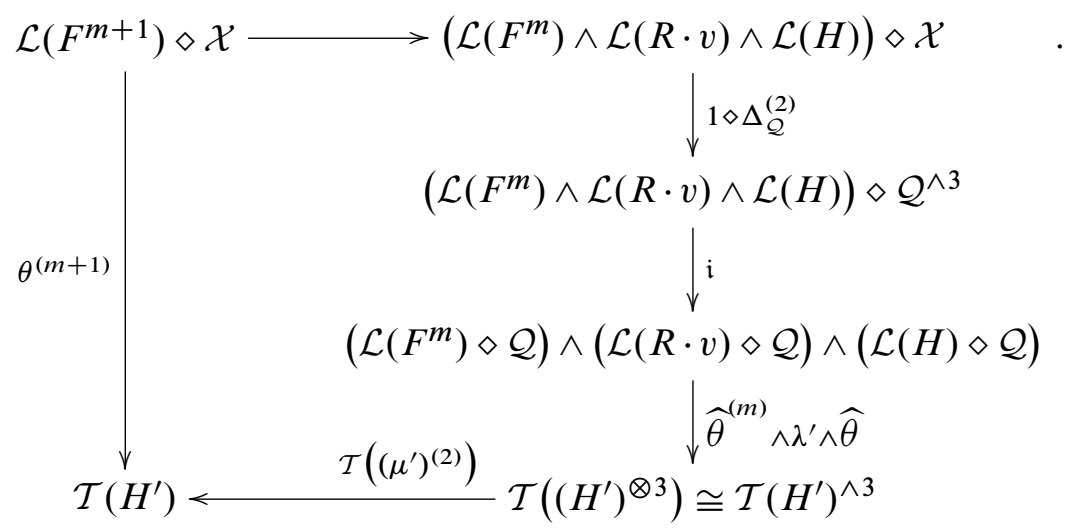

Here $\widehat{\theta}^{(m)}$ is slightly abusive shorthand for the composite

$$
\mathcal{L}\left(F^{m}\right) \diamond \mathcal{Q} \hookrightarrow \mathcal{T}\left(F^{m}\right) \diamond \mathcal{Q} \rightarrow \mathcal{T}\left(F^{m}\right) \diamond \mathcal{P} \mathcal{Q} \stackrel{\widehat{\theta}^{(m)}}{\longrightarrow} \mathcal{T}\left(H^{\prime}\right) .
$$

Applying Lemma 2.3, we obtain $\breve{\theta}^{(m+1)}: \mathcal{T}\left(F^{m+1}\right) \diamond \mathcal{X} \rightarrow \mathcal{T}\left(H^{\prime}\right)$. The composite

$$
\begin{aligned}
\mathcal{T}\left(F^{m+1}\right) \diamond \mathcal{Q} \cong \mathcal{T}\left(F^{m+1}\right) \diamond \mathcal{P} \diamond \mathcal{X} \diamond \mathcal{P} \stackrel{\rho \diamond 1 \diamond 1}{\longrightarrow} \mathcal{T}\left(F^{m+1}\right) \diamond \mathcal{X} \diamond \mathcal{P} \stackrel{\breve{\theta}^{(m+1)} \diamond \mathcal{P}}{\longrightarrow} \\
\mathcal{T}\left(H^{\prime}\right) \diamond \mathcal{P} \stackrel{\rho^{\prime}}{\longrightarrow} \mathcal{T}\left(H^{\prime}\right)
\end{aligned}
$$


then induces the desired map $\widehat{\theta}^{(m+1)}: \mathcal{T}\left(F^{m+1}\right) \diamond_{\mathcal{P}} \mathcal{Q} \rightarrow \mathcal{T}\left(H^{\prime}\right)$, since $\mathcal{Q}$ is a free bimodule. By construction, equality (A-1) now holds for all $k \leq m+1$ and $j \leq k$.

To complete the proof, set $\widehat{\theta}=\operatorname{colim}_{m} \widehat{\theta}^{(m)}$.

\section{A.2 Complete proof of Theorem 5.4(2)}

Recall that we have fixed $\mathfrak{F}(\Theta)=\left\{\theta_{k}: \Omega C \rightarrow H^{\otimes k} \mid k \geq 1\right\}$. Furthermore, for $k \geq 0$, we have defined

$$
\widetilde{\theta}_{k}: s^{-1}(C \oplus \bar{C}) \rightarrow\left(\Omega H \otimes_{t_{\Omega}} H\right) \otimes H^{\otimes k}
$$

by

$$
\widetilde{\theta}_{k}\left(s^{-1} c\right)=\left(\mathfrak{s} \otimes 1^{\otimes k}\right) \theta_{k+1}\left(s^{-1} c\right)
$$

and

$$
\widetilde{\theta}_{k}\left(s^{-1} \bar{c}\right)=\left(\mathfrak{h} \otimes 1^{\otimes k}\right) \theta_{k+1}\left(s^{-1} c\right),
$$

where 1 denotes the identity on $H$.

We claim that

$$
\left\{\widetilde{\theta}_{k}: s^{-1}(C \oplus \bar{C}) \rightarrow\left(\Omega H \otimes_{t_{\Omega}} H\right) \otimes H^{\otimes k} \mid k \geq 1\right\}
$$

satisfies the hypotheses of Proposition 3.16 and therefore induces a morphism

$$
\widetilde{\Theta} \in(\mathcal{A}, \mathcal{F})-\operatorname{PsHopf}\left(\mathfrak{P} \mathfrak{L}(C, \Psi), H \otimes_{t_{\Omega}} \Omega H\right),
$$

ie, $\widetilde{\theta}$ is a CASH map. We have already dealt with the case $k=0$, since $\widetilde{\theta}_{0}=\widetilde{\theta}$. Suppose that the claim is true for all $k<m$ and for $\widetilde{\theta}_{m}$ restricted to $s^{-1}(C \oplus \bar{C})_{<n}$.

Before proving the claim for $\widetilde{\theta}_{m}$ applied to $s^{-1}(C \oplus \bar{C})_{n}$, we establish some useful notation. For all $\mathbf{j} \in J_{k, m}$, let

$$
\widetilde{\theta}_{\mathbf{j}}=\widetilde{\theta}_{j_{0}} \otimes \theta_{j_{1}} \otimes \cdots \otimes \theta_{j_{k}} \text { and } \theta_{\mathbf{j}}=\theta_{j_{0}} \otimes \theta_{j_{1}} \otimes \cdots \otimes \theta_{j_{k}}
$$

as maps from $\Omega(C \oplus \bar{C}) \otimes \Omega C^{\otimes k}$ to $\left(\Omega H \otimes_{t_{\Omega}} H\right) \otimes H^{\otimes m}$ and from $\Omega C^{\otimes k+1}$ to $H^{\otimes m}$. Furthermore, let

$$
v^{\mathbf{j}}=v^{j_{0}} \otimes \chi^{\left(j_{1}-1\right)} \otimes \cdots \otimes \chi^{\left(j_{k}-1\right)} \text { and } \chi^{\mathbf{j}}=\chi^{\left(j_{0}-1\right)} \otimes \cdots \otimes \chi^{\left(j_{k}-1\right)}
$$

as maps from $\left(\Omega H \otimes_{t_{\Omega}} H\right) \otimes H^{\otimes k}$ to $\left(\Omega H \otimes t_{\Omega} H\right) \otimes H^{\otimes m}$ and from $H^{\otimes k+1}$ to $H^{\otimes m}$. Recall that $\chi$ is the comultiplication on $H$ and that $v$ is the right $\Omega C$-comodule action on $\mathfrak{P} \mathfrak{L}(C)$.

Algebraic $8 \mathcal{G}$ Geometric Topology, Volume 7 (2007) 
In terms of this notation $\widetilde{\theta}_{m}$ can be defined recursively by

$$
\widetilde{\theta}_{m} \mu=\mu\left(\sum_{\substack{0 \leq k \leq m \\ \mathbf{j} \in J_{k, m}}} v^{\mathbf{j}} \widetilde{\theta}_{k} \otimes \widetilde{\theta}_{\mathbf{j}} v^{(k)}\right),
$$

where all multiplication maps are denoted $\mu$ and all coactions are denoted $\nu$.

Observe that since $\mathfrak{s}$ and $\mathfrak{h}$ are maps of comodules, $v^{(j)} \mathfrak{s}=\left(\mathfrak{s} \otimes 1^{\otimes j}\right) \chi^{(j-1)}$ and $v^{(j)} \mathfrak{h}=\left(\mathfrak{h} \otimes 1^{\otimes j}\right) \chi^{(j-1)}$ for all $j$.

Let $s^{-1} c \in s^{-1}(C \oplus \bar{C})_{n}$. Let $c_{i} \otimes c^{i}$ denote the image of $c$ under the reduced comultiplication in $C$. Then

$$
\begin{aligned}
& \widetilde{\theta}_{m} d_{\Omega}\left(s^{-1} c\right) \\
& =\widetilde{\theta}_{m}\left(-s^{-1}(d c)+s^{-1} \bar{c}+(-1)^{c_{i}} s^{-1} c_{i} s^{-1} c^{i}\right) \\
& =-\left(\mathfrak{s} \otimes 1^{\otimes m}\right) \theta_{m+1}\left(s^{-1}(d c)\right)+\left(\mathfrak{h} \otimes 1^{\otimes m}\right) \theta_{m+1}\left(s^{-1} c\right) \\
& +(-1)^{c_{i}} \mu\left(\sum_{\substack{0 \leq k \leq m \\
\mathbf{j} \in J_{k, m}}} v^{\mathbf{j}} \widetilde{\theta}_{k}\left(s^{-1} c_{i}\right) \otimes \widetilde{\theta}_{\mathbf{j}} v^{(k)}\left(s^{-1} c^{i}\right)\right) \\
& =-\left(\mathfrak{s} \otimes 1^{\otimes m}\right) \theta_{m+1}\left(s^{-1}(d c)\right)+\left(\mathfrak{h} \otimes 1^{\otimes m}\right) \theta_{m+1}\left(s^{-1} c\right) \\
& +(-1)^{c_{i}} \mu\left(\sum_{\substack{0 \leq k \leq m \\
\mathbf{j} \in J_{k, m}}} \nu^{\mathbf{j}}\left(\mathfrak{s} \otimes 1^{\otimes k}\right) \theta_{k}\left(s^{-1} c_{i}\right) \otimes \widetilde{\theta}_{\mathbf{j}} \psi^{(k)}\left(s^{-1} c^{i}\right)\right) \\
& =-\left(\mathfrak{s} \otimes 1^{\otimes m}\right) \theta_{m+1}\left(s^{-1}(d c)\right)+\left(\mathfrak{h} \otimes 1^{\otimes m}\right) \theta_{m+1}\left(s^{-1} c\right) \\
& +(-1)^{c_{i}} \mu\left(\sum_{\substack{0 \leq k \leq m \\
\mathbf{j} \in J_{k, m}}}\left(\mathfrak{s} \otimes 1^{\otimes m}\right) \chi^{\mathbf{j}} \theta_{k}\left(s^{-1} c_{i}\right) \otimes\left(\mathfrak{s} \otimes 1^{\otimes m}\right) \theta_{\mathbf{j}} \psi^{(k)}\left(s^{-1} c^{i}\right)\right) \\
& =-\left(\mathfrak{s} \otimes 1^{\otimes m}\right) \theta_{m+1}\left(s^{-1}(d c)\right)+\left(\mathfrak{h} \otimes 1^{\otimes m}\right) \theta_{m+1}\left(s^{-1} c\right) \\
& +(-1)^{c_{i}}\left(\mathfrak{s} \otimes 1^{\otimes m}\right) \mu\left(\sum_{\substack{0 \leq k \leq m \\
\mathbf{j} \in J_{k, m}}} \chi^{\mathbf{j}} \theta_{k}\left(s^{-1} c_{i}\right) \otimes \theta_{\mathbf{j}} \psi^{(k)}\left(s^{-1} c^{i}\right)\right) \\
& =-\left(\mathfrak{s} \otimes 1^{\otimes m}\right) \theta_{m+1}\left(s^{-1}(d c)\right)+\left(\mathfrak{h} \otimes 1^{\otimes m}\right) \theta_{m+1}\left(s^{-1} c\right) \\
& +(-1)^{c_{i}}\left(\mathfrak{s} \otimes 1^{\otimes m}\right) \theta_{m+1}\left(s^{-1} c_{i} \cdot s^{-1} s^{i}\right) \\
& =\left(\mathfrak{s} \otimes 1^{\otimes m}\right) \theta_{m+1} d_{\Omega}\left(s^{-1} c\right)+\left(\mathfrak{h} \otimes 1^{\otimes m}\right) \theta_{m+1}\left(s^{-1} c\right) \text {. }
\end{aligned}
$$

Algebraic 83 Geometric Topology, Volume 7 (2007) 
Next,

$$
\begin{aligned}
\left(D_{\Omega} \otimes 1^{\otimes m}\right) \widetilde{\theta}_{m}\left(s^{-1} c\right) & =\left(D_{\Omega} \mathfrak{s} \otimes 1^{\otimes m}\right) \theta_{m+1}\left(s^{-1} c\right) \\
& =\left(\mathfrak{s} d \otimes 1^{\otimes m}\right) \theta_{m+1}\left(s^{-1} c\right)+\left(\mathfrak{h} \otimes 1^{\otimes m}\right) \theta_{m+1}\left(s^{-1} c\right) \\
& =\left(\mathfrak{s} \otimes 1^{\otimes m}\right)\left(d \otimes 1^{\otimes m}\right) \theta_{m+1}\left(s^{-1} c\right)+\left(\mathfrak{h} \otimes 1^{\otimes m}\right) \theta_{m+1}\left(s^{-1} c\right)
\end{aligned}
$$

and

$$
\begin{aligned}
\left(1 \otimes \sum_{r+s=m-1} 1^{\otimes r} \otimes d \otimes 1^{\otimes s}\right) \widetilde{\theta}_{m}\left(s^{-1} c\right) & \left(\mathfrak{s} \otimes \sum_{r+s=m-1} 1^{\otimes r} \otimes d \otimes 1^{\otimes s}\right) \theta_{m+1}\left(s^{-1} c\right) \\
& =\left(\mathfrak{s} \otimes 1^{\otimes m}\right)\left(\sum_{r+s=m-1}\left(1^{\otimes r+1} \otimes d \otimes 1^{\otimes s}\right) \theta_{m+1}\left(s^{-1} c\right)\right) .
\end{aligned}
$$

On the other hand

$$
\begin{aligned}
\left(v \otimes 1^{\otimes m-1}\right) \widetilde{\theta}_{m-1}\left(s^{-1} c\right) & =\left(v \mathfrak{s} \otimes 1^{\otimes m-1}\right) \theta_{m}\left(s^{-1} c\right) \\
& =\left(\mathfrak{s} \otimes 1^{\otimes m}\right)\left(\chi \otimes 1^{\otimes m-1}\right) \theta_{m}\left(s^{-1} c\right)
\end{aligned}
$$

and

$$
\begin{aligned}
\left(1 \otimes \sum_{r+s=m-2} 1^{\otimes r} \otimes \chi \otimes 1^{\otimes s}\right) & \widetilde{\theta}_{m-1}\left(s^{-1} c\right) \\
& =\left(\mathfrak{s} \otimes \sum_{r+s=m-2} 1^{\otimes r} \otimes \chi \otimes 1^{\otimes s}\right) \theta_{m}\left(s^{-1} c\right) \\
& =\left(\mathfrak{s} \otimes 1^{\otimes m}\right)\left(\sum_{r+s=m-1}\left(1^{\otimes r} \otimes \chi \otimes 1^{\otimes s}\right) \theta_{m}\left(s^{-1} c\right)\right) .
\end{aligned}
$$

Finally

$$
\begin{aligned}
& \sum_{r+s=m}\left(\widetilde{\theta}_{r} \otimes \theta_{s}\right) v\left(s^{-1} c\right)=\sum_{r+s=m}\left(\widetilde{\theta}_{r} \otimes \theta_{s}\right)(1 \otimes \Omega \pi) \widetilde{\psi}\left(s^{-1} c\right) \\
& =\sum_{r+s=m}\left(\widetilde{\theta}_{r} \otimes \theta_{s}\right)(1 \otimes \Omega \pi) \psi\left(s^{-1} c\right) \\
& =\sum_{r+s=m}\left(\widetilde{\theta}_{r} \otimes \theta_{s}\right) \psi\left(s^{-1} c\right) \\
& =\sum_{r+s=m}\left(\left(\mathfrak{s} \otimes 1^{\otimes r}\right) \theta_{r+1} \otimes \theta_{s}\right) \psi\left(s^{-1} c\right) \\
& =\left(\mathfrak{s} \otimes 1^{\otimes m}\right)\left(\sum_{r+s=m}\left(\theta_{r+1} \otimes \theta_{s}\right) \psi\left(s^{-1} c\right)\right) .
\end{aligned}
$$

Algebraic 83 Geometric Topology, Volume 7 (2007) 
The sum of the terms above, with appropriate signs, yields $\mathfrak{s} \otimes 1^{\otimes m}$ applied to the difference of the two sides of equation (3-3) for $\theta_{m+1}$. The sum is therefore zero, as desired, ie, the hypothesis of Proposition 3.16 holds for $s^{-1} c \in s^{-1}(C \oplus \bar{C})_{n}$.

Before showing that the same condition holds for the remaining generators, we consider the relation between the degree -1 map $\kappa: \Omega C \rightarrow \Omega(C \oplus \bar{C})$ of Lemma 4.8 and $\widetilde{\theta}_{k}$. Note that $\widetilde{\theta}_{k}$ has been defined precisely so that

$$
\widetilde{\theta}_{k} \circ \kappa=\left(\mathfrak{h} \otimes 1^{\otimes k}\right) \theta_{k+1}: s^{-1} C \rightarrow\left(\Omega H \otimes_{t_{\Omega}} H\right) \otimes H^{\otimes k}
$$

for all $k$. We show now by induction on $k$ and on wordlength in $\Omega C$ that this equality holds in fact on all of $\Omega C$, for all $k$.

Suppose that $\widetilde{\theta}_{k} \kappa=\left(\mathfrak{h} \otimes 1^{\otimes k}\right) \theta_{k+1}$ everywhere in $\Omega C$ for all $k<m$ and that $\widetilde{\theta}_{m} \kappa=\left(\mathfrak{h} \otimes 1^{\otimes m}\right) \theta_{m+1}$ on $T^{<\ell} s^{-1} C_{+}$. Then on $\bigoplus_{a+b=\ell} T^{a} s^{-1} C_{+} \otimes T^{b} s^{-1} C_{+}$ we have that

$$
\begin{aligned}
\widetilde{\theta}_{m} \kappa \mu & =\widetilde{\theta}_{m} \mu(\kappa \otimes \iota+\iota \otimes \kappa) \\
& =\mu\left(\sum_{\substack{1 \leq k \leq m \\
\mathbf{j} \in J_{k, m}}}\left(v^{\mathbf{j}} \widetilde{\theta}_{k} \kappa \otimes \widetilde{\theta}_{\mathbf{j}} v^{(k)} \iota+v^{\mathbf{j}} \widetilde{\theta}_{k} \iota \otimes \widetilde{\theta}_{\mathbf{j}} v^{(k)} \kappa\right)\right) \\
& =\mu\left(\sum _ { \substack { 1 \leq k \leq m \\
\mathbf { j } \in J _ { k , m } } } \left(v^{\mathbf{j}}\left(\mathfrak{h} \otimes 1^{\otimes k}\right) \theta_{k+1} \otimes\left(\mathfrak{s} \otimes 1^{\otimes m}\right) \theta_{\mathbf{j}} \psi^{(k)}\right.\right. \\
& \left.\left.+\left(\mathfrak{s} \otimes 1^{\otimes m}\right) \chi^{\mathbf{j}} \theta_{k+1} \otimes \widetilde{\theta}_{\mathbf{j}}\left(\kappa \otimes 1^{\otimes k}\right) \psi^{(k)}\right)\right) \\
& =\mu\left(\sum _ { \substack { 1 \leq k \leq m \\
\mathbf { j } \in J _ { k , m } } } \left(\left(\mathfrak{h} \otimes 1^{\otimes m}\right) \chi^{\mathbf{j}} \theta_{k+1} \otimes\left(\mathfrak{s} \otimes 1^{\otimes m}\right) \theta_{\mathbf{j}} \psi^{(k)}\right.\right. \\
& \left.\left.=\left(\mathfrak{h} \otimes 1^{\otimes m}\right) \chi^{\mathbf{j}} \theta_{k+1} \otimes\left(\mathfrak{h} \otimes 1^{\otimes m}\right) \theta_{\mathbf{j}} \psi^{(k)}\right)\right) \\
& \left(\sum_{\substack{1 \leq k \leq m \\
\mathbf{j} \in J_{k, m}}}\left(\chi^{\mathbf{j}} \theta_{k+1} \otimes \theta_{\mathbf{j}} \psi^{(k)}+\chi^{\mathbf{j}} \theta_{k+1} \otimes \theta_{\mathbf{j}} \psi^{(k)}\right)\right) \\
& =\left(\mathfrak{h} \otimes 1^{\otimes m}\right) \theta_{m+1} \mu .
\end{aligned}
$$

Thus $\widetilde{\theta}_{m} \kappa=\left(\mathfrak{h} \otimes 1^{\otimes m}\right) \theta_{m+1}$ on $T^{\leq \ell} S^{-1} C_{+}$.

We can therefore conclude by induction that

$$
\widetilde{\theta}_{m} \kappa=\left(\mathfrak{h} \otimes 1^{\otimes m}\right) \theta_{m+1}: \Omega C \rightarrow\left(\Omega H \otimes_{t_{\Omega}} H\right) \otimes H^{\otimes m}
$$

Algebraic $8 \mathcal{G}$ Geometric Topology, Volume 7 (2007) 
for all $m$.

Suppose now that $s^{-1} \bar{c} \in s^{-1}(C \oplus \bar{C})_{n}$. Let $c_{i} \otimes c^{i}$ denote the image of $c$ under the reduced comultiplication in $C$. Then

$$
\begin{aligned}
& \widetilde{\theta}_{m} d_{\Omega}\left(s^{-1} \bar{c}\right)=\widetilde{\theta}_{m}\left(s^{-1}(\overline{d c})-(-1)^{c_{i}} s^{-1} \bar{c}_{i} s^{-1} c^{i}+s^{-1} c_{i} s^{-1} \bar{c}^{i}\right) \\
& =\left(\mathfrak{h} \otimes 1^{\otimes m}\right) \theta_{m+1}\left(s^{-1}(d c)\right) \\
& -(-1)^{c_{i}} \mu\left(\sum_{\substack{0 \leq k \leq m \\
\mathbf{j} \in J_{k, m}}} v^{\mathbf{j}} \widetilde{\theta}_{k}\left(s^{-1} \bar{c}_{i}\right) \otimes \widetilde{\theta}_{\mathbf{j}} v^{(k)}\left(s^{-1} c^{i}\right)\right) \\
& +\mu\left(\sum_{\substack{0 \leq k \leq m \\
\mathbf{j} \in J_{k, m}}} v^{\mathbf{j}} \widetilde{\theta}_{k}\left(s^{-1} c_{i}\right) \otimes \widetilde{\theta}_{\mathbf{j}} v^{(k)}\left(s^{-1} \bar{c}^{i}\right)\right) \\
& =\left(\mathfrak{h} \otimes 1^{\otimes m}\right) \theta_{m+1}\left(s^{-1}(d c)\right) \\
& -(-1)^{c_{i}} \mu\left(\sum_{\substack{0 \leq k \leq m \\
\mathbf{j} \in J_{k, m}}} v^{\mathbf{j}}\left(\mathfrak{h} \otimes 1^{\otimes k}\right) \theta_{k}\left(s^{-1} c_{i}\right) \otimes \widetilde{\theta}_{\mathbf{j}} \psi^{(k)}\left(s^{-1} c^{i}\right)\right) \\
& +\mu\left(\sum_{\substack{0 \leq k \leq m \\
\mathbf{j} \in J_{k, m}}} v^{\mathbf{j}}\left(\mathfrak{s} \otimes 1^{\otimes k}\right) \theta_{k}\left(s^{-1} c_{i}\right) \otimes \widetilde{\theta}_{\mathbf{j}}\left(\kappa \otimes 1^{\otimes k}\right) \psi^{(k)}\left(s^{-1} c^{i}\right)\right) \\
& =\left(\mathfrak{h} \otimes 1^{\otimes m}\right) \theta_{m+1}\left(s^{-1}(d c)\right) \\
& -(-1)^{c_{i}} \mu\left(\sum_{\substack{0 \leq k \leq m \\
\mathbf{j} \in J_{k, m}}}\left(\mathfrak{h} \otimes 1^{\otimes m}\right) \chi^{\mathbf{j}} \theta_{k}\left(s^{-1} c_{i}\right) \otimes\left(\mathfrak{s} \otimes 1^{\otimes m}\right) \theta_{\mathbf{j}} \psi^{(k)}\left(s^{-1} c^{i}\right)\right) \\
& +\mu\left(\sum_{\substack{0 \leq k \leq m \\
\mathbf{j} \in J_{k, m}}}\left(\mathfrak{s} \otimes 1^{\otimes m}\right) \chi^{\mathbf{j}} \theta_{k}\left(s^{-1} c_{i}\right) \otimes\left(\mathfrak{h} \otimes 1^{\otimes m}\right) \theta_{\mathbf{j}} \psi^{(k)}\left(s^{-1} c^{i}\right)\right) \\
& =\left(\mathfrak{h} \otimes 1^{\otimes m}\right) \theta_{m+1}\left(s^{-1}(d c)\right) \\
& +\left(\mathfrak{h} \otimes 1^{\otimes m}\right) \mu\left(\sum_{\substack{0 \leq k \leq m \\
\mathbf{j} \in J_{k, m}}} \chi^{\mathbf{j}} \theta_{k}\left(s^{-1} c_{i}\right) \otimes \theta_{\mathbf{j}} \psi^{(k)}\left(s^{-1} c^{i}\right)\right) \\
& =\left(\mathfrak{h} \otimes 1^{\otimes m}\right) \theta_{m+1}\left(s^{-1}(d c)\right)+\left(\mathfrak{h} \otimes 1^{\otimes m}\right) \theta_{m+1}\left(s^{-1} c_{i} s^{-1} c^{i}\right) \\
& =\left(\mathfrak{h} \otimes 1^{\otimes m}\right) \theta_{m+1} d_{\Omega}\left(s^{-1} c\right) \text {. }
\end{aligned}
$$

Algebraic $\mathcal{E} \mathcal{G}$ Geometric Topology, Volume 7 (2007) 
Next,

$$
\begin{aligned}
\left(D_{\Omega} \otimes 1^{\otimes m}\right) \widetilde{\theta}_{m}\left(s^{-1} \bar{c}\right) & =\left(D_{\Omega} \mathfrak{h} \otimes 1^{\otimes m}\right) \theta_{m+1}\left(s^{-1} c\right) \\
& =-\left(\mathfrak{h} d \otimes 1^{\otimes m}\right) \theta_{m+1}\left(s^{-1} c\right) \\
& =-\left(\mathfrak{h} \otimes 1^{\otimes m}\right)\left(d \otimes 1^{\otimes m}\right) \theta_{m+1}\left(s^{-1} c\right)
\end{aligned}
$$

and

$$
\begin{aligned}
\left(1 \otimes \sum_{r+s=m-1} 1^{\otimes r}\right. & \left.\otimes d \otimes 1^{\otimes s}\right) \widetilde{\theta}_{m}\left(s^{-1} \bar{c}\right) \\
& =\left(\mathfrak{h} \otimes \sum_{r+s=m-1} 1^{\otimes r} \otimes d \otimes 1^{\otimes s}\right) \theta_{m+1}\left(s^{-1} c\right) \\
& =\left(\mathfrak{h} \otimes 1^{\otimes m}\right)\left(\sum_{r+s=m-1}\left(1^{\otimes r+1} \otimes d \otimes 1^{\otimes s}\right) \theta_{m+1}\left(s^{-1} c\right)\right) .
\end{aligned}
$$

On the other hand,

$$
\begin{aligned}
\left(\nu \otimes 1^{\otimes m-1}\right) \widetilde{\theta}_{m-1}\left(s^{-1} \bar{c}\right) & =\left(\nu \mathfrak{h} \otimes 1^{\otimes m-1}\right) \theta_{m}\left(s^{-1} c\right) \\
& =\left(\mathfrak{h} \otimes 1^{\otimes m}\right)\left(\chi \otimes 1^{\otimes m-1}\right) \theta_{m}\left(s^{-1} c\right)
\end{aligned}
$$

and

$$
\begin{aligned}
(1 \otimes & \left.\sum_{r+s=m-2} 1^{\otimes r} \otimes \chi \otimes 1^{\otimes s}\right) \widetilde{\theta}_{m-1}\left(s^{-1} \bar{c}\right) \\
& =\left(\mathfrak{h} \otimes \sum_{r+s=m-2} 1^{\otimes r} \otimes \chi \otimes 1^{\otimes s}\right) \theta_{m}\left(s^{-1} c\right) \\
& =\left(\mathfrak{h} \otimes 1^{\otimes m}\right)\left(\sum_{r+s=m-1}\left(1^{\otimes r} \otimes \chi \otimes 1^{\otimes s}\right) \theta_{m}\left(s^{-1} c\right)\right) .
\end{aligned}
$$

Finally

$$
\begin{aligned}
\sum_{r+s=m}\left(\widetilde{\theta}_{r} \otimes \theta_{s}\right) v\left(s^{-1} \bar{c}\right) & =\sum_{r+s=m}\left(\widetilde{\theta}_{r} \otimes \theta_{s}\right) \nu \kappa\left(s^{-1} c\right) \\
& =\sum_{r+s=m}\left(\widetilde{\theta}_{r} \otimes \theta_{s}\right)(\kappa \otimes 1) \psi\left(s^{-1} c\right) \\
& =\sum_{r+s=m}\left(\left(\mathfrak{h} \otimes 1^{\otimes r}\right) \theta_{r+1} \otimes \theta_{s}\right) \psi\left(s^{-1} c\right) \\
& =\left(\mathfrak{h} \otimes 1^{\otimes m}\right)\left(\sum_{r+s=m}\left(\theta_{r+1} \otimes \theta_{s}\right) \psi\left(s^{-1} c\right)\right) .
\end{aligned}
$$

Algebraic 83 Geometric Topology, Volume 7 (2007) 
The sum of the above terms, with appropriate signs, yields $\mathfrak{h} \otimes 1^{\otimes m}$ applied to the difference of the two sides of equation (3-3) for $\theta_{m+1}$. The sum is therefore zero, as desired, ie, the hypothesis of Proposition 3.16 holds for $s^{-1} \bar{c} \in s^{-1}(C \oplus \bar{C})_{n}$.

Since we can prove by induction that (3-3) holds for all $m$, we can conclude that $\widetilde{\theta}$ is indeed a CASH map.

\section{References}

[1] H-J Baues, The cobar construction as a Hopf algebra, Invent. Math. 132 (1998) 467-489 MR1625728

[2] F R Cohen, J C Moore, J A Neisendorfer, Torsion in homotopy groups, Ann. of Math. (2) 109 (1979) 121-168 MR519355

[3] S Eilenberg, J C Moore, Homology and fibrations. I. Coalgebras, cotensor product and its derived functors, Comment. Math. Helv. 40 (1966) 199-236 MR0203730

[4] Y Félix, S Halperin, J-C Thomas, Differential graded algebras in topology, from: "Handbook of algebraic topology", North-Holland, Amsterdam (1995) 829-865 MR1361901

[5] V K A M Gugenheim, H J Munkholm, On the extended functoriality of Tor and Cotor, J. Pure Appl. Algebra 4 (1974) 9-29 MR0347946

[6] K Hess, P-E Parent, J Scott, Co-rings over operads characterize morphisms arXiv: math.AT/0505559

[7] K Hess, P-E Parent, J Scott, A chain coalgebra model for the James map, Homology, Homotopy Appl. 9 (2007)

[8] K Hess, P-E Parent, J Scott, A Tonks, A canonical enriched Adams-Hilton model for simplicial sets, Adv. Math. 207 (2006) 847-875 MR2271989

[9] M Markl, Operads and PROPs arXiv:math. AT/0601129

[10] M Markl, S Shnider, J Stasheff, Operads in algebra, topology and physics, Mathematical Surveys and Monographs 96, American Mathematical Society, Providence, RI (2002) MR1898414

[11] R J Milgram, Iterated loop spaces, Ann. of Math. (2) 84 (1966) 386-403 MR0206951

[12] H R Miller, A localization theorem in homological algebra, Math. Proc. Cambridge Philos. Soc. 84 (1978) 73-84 MR0494105

[13] J W Milnor, J C Moore, On the structure of Hopf algebras, Ann. of Math. (2) 81 (1965) 211-264 MR0174052

[14] B Ndombol, J-C Thomas, On the cohomology algebra of free loop spaces, Topology 41 (2002) 85-106 MR1871242 
[15] J Stasheff, S Halperin, Differential algebra in its own rite, from: "Proceedings of the Advanced Study Institute on Algebraic Topology (Aarhus Univ., Aarhus 1970), Vol. III", Mat. Inst., Aarhus Univ., Aarhus (1970) 567-577. Various Publ. Ser., No. 13 MR0339181

[16] R H Szczarba, The homology of twisted cartesian products, Trans. Amer. Math. Soc. 100 (1961) 197-216 MR0137111

[17] D Tanré, Homotopie rationnelle: modèles de Chen, Quillen, Sullivan, Lecture Notes in Mathematics 1025, Springer, Berlin (1983) MR764769

Institut de géométrie, algèbre et topologie (IGAT), École Polytechnique Fédérale de Lausanne, CH-1015 Lausanne, Switzerland

Department of Mathematical Sciences, King's College, University of Aberdeen, Aberdeen AB24 3UE, UK

kathryn.hess@epfl.ch, ran@maths.abdn.ac.uk

http://sma.epfl.ch/ hessbell/, http://www.maths.abdn.ac.uk/ ran/

Received: 26 April 2007

Algebraic $\mathcal{B}$ Geometric Topology, Volume 7 (2007) 